\title{
Electrophilicity of 5-Benzylidene-1,3-
}

\section{dimethylbarbituric and thiobarbituric acids}

Florian Seeliger, Stefan T. A. Berger, Grygoriy Y. Remennikov, Kurt Polborn and Herbert Mayr*

Department Chemie und Biochemie, Ludwig-Maximilians-Universität München, Butenandtstr. 5-13, 81377 München, Germany

table of contents

Kinetic experiments 2

reactions of electrophile $\mathbf{1 a}$ reactions of electrophile $\mathbf{1 b}$ reactions of electrophile 1c reactions of electrophile $\mathbf{1 d}$ 2 4 reactions of electrophile $\mathbf{1 e}$

NMR spectra

nmr of products 1

nmr of products $\mathbf{3}$

nmr of products 4

nmr of products 5

$\mathrm{nmr}$ of $\mathbf{8 c h}$

$\mathrm{nmr}$ of $9 \mathrm{ch}$

nmr of products $\mathbf{1 1}$

nmr of 12cc

nmr of 14ca 


\section{Kinetic experiments}

The temperature of the solutions during all kinetic studies was kept constant $\left(20 \pm 0.1^{\circ} \mathrm{C}\right)$ by using a circulating bath thermostat. Dry DMSO for kinetics was purchased $\left(<50 \mathrm{ppm}_{2} \mathrm{O}\right)$. For the evaluation of kinetics the stopped-flow spectrophotometer systems Hi-Tech SF61DX2 or Applied Photophysics SX.18MV-R stopped-flow reaction analyzer were used. Rate constants $k_{\mathrm{obs}}\left(\mathrm{s}^{-1}\right)$ were obtained by fitting the single exponential $A_{\mathrm{t}}=A_{0} \exp \left(-k_{\mathrm{obs}} t\right)+C$ to the observed time-dependent electrophile absorbance (averaged from at least 4 kinetic runs for each nucleophile concentration). For the stopped-flow experiments 2 stock solutions were used: A solution of the electrophile in DMSO and a solution of the carbanion, which was either used as potassium salt or generated by the deprotonation of the $\mathrm{CH}$ acid with 1.05 equivalents of $\mathrm{KO} t \mathrm{Bu}$.

\section{reactions of electrophile $1 \mathrm{a}$}

reaction of $\mathbf{1 a}$ with $\mathbf{2 b}$

(DMSO, $20{ }^{\circ} \mathrm{C}$, stopped flow, $487 \mathrm{~nm}$ )

\begin{tabular}{ccc}
\hline$[\mathbf{1 a}] / \mathrm{M}$ & {$[\mathbf{2 b}-\mathrm{K}] / \mathrm{M}$} & $k_{\text {obs }} / \mathrm{s}^{-1}$ \\
\hline $2.18 \times 10^{-5}$ & $7.78 \times 10^{-4}$ & $1.24 \times 10^{-1}$ \\
$2.18 \times 10^{-5}$ & $1.56 \times 10^{-3}$ & $2.46 \times 10^{-1}$ \\
$2.18 \times 10^{-5}$ & $2.22 \times 10^{-3}$ & $3.53 \times 10^{-1}$ \\
$2.18 \times 10^{-5}$ & $3.00 \times 10^{-3}$ & $4.60 \times 10^{-1}$ \\
$2.18 \times 10^{-5}$ & $3.89 \times 10^{-3}$ & $5.89 \times 10^{-1}$ \\
\hline$k_{2}=(1.49 \pm 0.03) \times 10^{2} \mathrm{M}^{-1} \mathrm{~s}^{-1}$ &
\end{tabular}

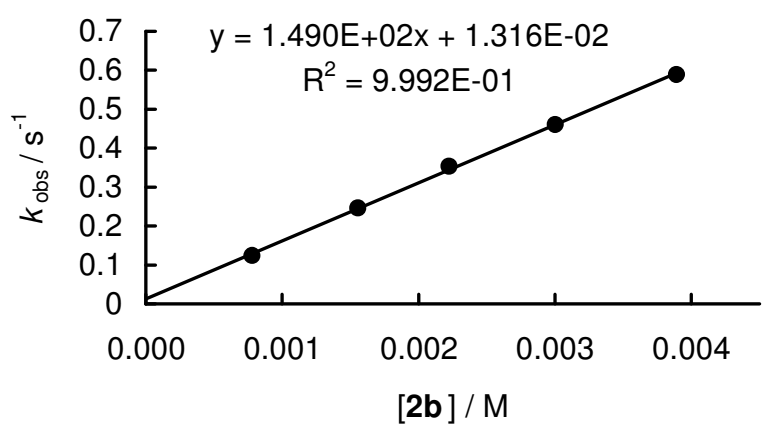

reaction of $\mathbf{1 a}$ with $\mathbf{2 c}$ (DMSO, $20{ }^{\circ} \mathrm{C}$, stopped flow, $480 \mathrm{~nm}$ )

\begin{tabular}{ccc}
\hline$[\mathbf{1 a}] / \mathrm{M}$ & {$[\mathbf{2 c}] / \mathrm{M}$} & $k_{\text {obs }} / \mathrm{s}^{-1}$ \\
\hline $2.39 \times 10^{-5}$ & $4.09 \times 10^{-4}$ & $3.37 \times 10^{-2}$ \\
$2.39 \times 10^{-5}$ & $8.18 \times 10^{-4}$ & $5.16 \times 10^{-2}$ \\
$2.39 \times 10^{-5}$ & $9.20 \times 10^{-4}$ & $5.80 \times 10^{-2}$ \\
$2.39 \times 10^{-5}$ & $1.23 \times 10^{-3}$ & $7.55 \times 10^{-2}$ \\
$2.39 \times 10^{-5}$ & $1.64 \times 10^{-3}$ & $9.90 \times 10^{-2}$ \\
\hline$k_{2}=(5.37 \pm 0.21) \times 10^{1} \mathrm{M}^{-1} \mathrm{~s}^{-1}$ &
\end{tabular}

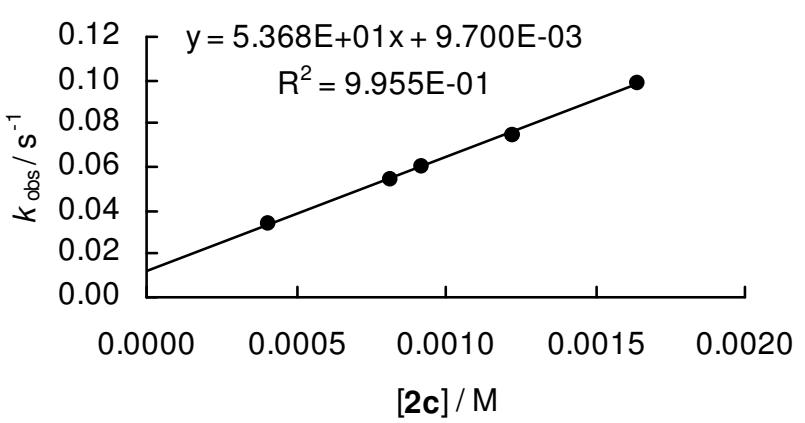


reaction of $\mathbf{1 a}$ with $\mathbf{2 d}$

(DMSO, $20{ }^{\circ} \mathrm{C}$, stopped flow, $520 \mathrm{~nm}$ )

\begin{tabular}{ccc}
\hline$[\mathbf{1 a}] / \mathrm{M}$ & {$[\mathbf{2 d}] / \mathrm{M}$} & $k_{\text {obs }} / \mathrm{s}^{-1}$ \\
\hline $1.60 \times 10^{-5}$ & $2.27 \times 10^{-4}$ & $3.52 \times 10^{-2}$ \\
$1.60 \times 10^{-5}$ & $4.54 \times 10^{-4}$ & $7.30 \times 10^{-2}$ \\
$1.60 \times 10^{-5}$ & $6.81 \times 10^{-4}$ & $1.23 \times 10^{-1}$ \\
$1.60 \times 10^{-5}$ & $9.08 \times 10^{-4}$ & $1.70 \times 10^{-1}$ \\
$1.60 \times 10^{-5}$ & $1.13 \times 10^{-3}$ & $2.00 \times 10^{-1}$ \\
\hline$k_{2}=(1.88 \pm 0.08) \times 10^{2} \mathrm{M}^{-1} \mathrm{~s}^{-1}$ &
\end{tabular}

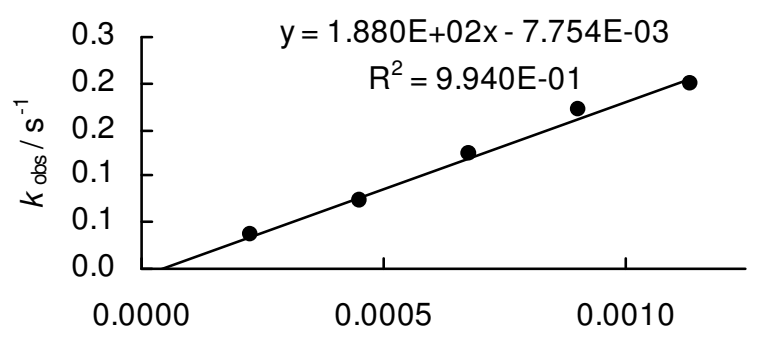

$[\mathbf{2 d}] / \mathrm{M}$

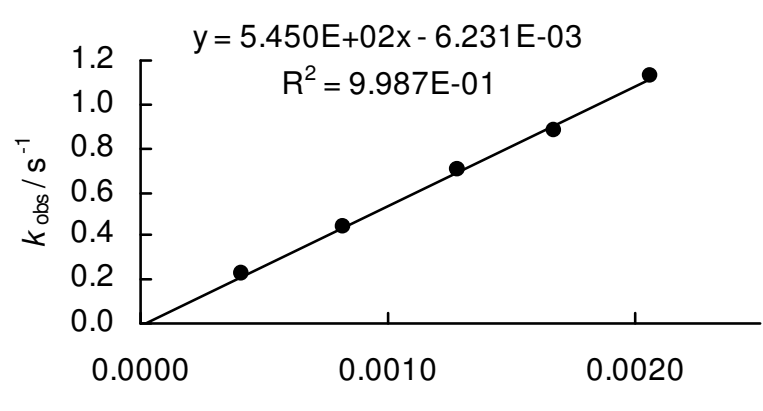

$[2 \mathrm{e}] / \mathrm{M}$

reaction of $1 \mathrm{a}$ with $\mathbf{2 f}$

(DMSO, $20{ }^{\circ} \mathrm{C}$, stopped flow, $480 \mathrm{~nm}$ )

\begin{tabular}{ccc}
\hline$[1 \mathrm{a}] / \mathrm{M}$ & {$[\mathbf{2 f}] / \mathrm{M}$} & $k_{\mathrm{obs}} / \mathrm{s}^{-1}$ \\
\hline $2.08 \times 10^{-5}$ & $4.18 \times 10^{-4}$ & $2.96 \times 10^{-1}$ \\
$2.08 \times 10^{-5}$ & $8.37 \times 10^{-4}$ & $7.45 \times 10^{-1}$ \\
$2.08 \times 10^{-5}$ & $1.19 \times 10^{-3}$ & 1.01 \\
$2.08 \times 10^{-5}$ & $1.67 \times 10^{-3}$ & 1.59 \\
$2.08 \times 10^{-5}$ & $2.15 \times 10^{-3}$ & 2.04 \\
\hline$k_{2}=(1.01 \pm 0.03) \times 10^{3} \mathrm{M}^{-1} \mathrm{~s}^{-1}$ &
\end{tabular}

reaction of $\mathbf{1 a}$ with $\mathbf{2 g}$

(DMSO, $20{ }^{\circ} \mathrm{C}$, stopped flow, $500 \mathrm{~nm}$ )

\begin{tabular}{ccc}
\hline$[\mathbf{1 a}] / \mathrm{M}$ & {$[\mathbf{2 g}] / \mathrm{M}$} & $k_{\mathrm{obs}} / \mathrm{s}^{-1}$ \\
\hline $2.90 \times 10^{-5}$ & $4.08 \times 10^{-4}$ & 1.48 \\
$2.90 \times 10^{-5}$ & $8.17 \times 10^{-4}$ & 2.99 \\
$2.90 \times 10^{-5}$ & $1.14 \times 10^{-3}$ & 4.33 \\
$2.90 \times 10^{-5}$ & $1.43 \times 10^{-3}$ & 5.29 \\
$2.90 \times 10^{-5}$ & $1.76 \times 10^{-3}$ & 6.58 \\
\hline \multicolumn{2}{l}{$k_{2}=(3.78 \pm 0.05) \times 10^{3} \mathrm{M}^{-1} \mathrm{~s}^{-1}$}
\end{tabular}

reaction of $1 \mathrm{a}$ with $2 \mathrm{~h}$

(DMSO, $20^{\circ} \mathrm{C}$, stopped flow, $487 \mathrm{~nm}$ )

\begin{tabular}{ccc}
\hline$[1 \mathrm{a}] / \mathrm{M}$ & {$[\mathbf{2 h}] / \mathrm{M}$} & $k_{\text {obs }} / \mathrm{s}^{-1}$ \\
\hline $2.18 \times 10^{-5}$ & $9.51 \times 10^{-4}$ & $1.14 \times 10^{1}$ \\
$2.18 \times 10^{-5}$ & $1.43 \times 10^{-3}$ & $1.75 \times 10^{1}$ \\
$2.18 \times 10^{-5}$ & $1.90 \times 10^{-3}$ & $2.31 \times 10^{1}$ \\
$2.18 \times 10^{-5}$ & $2.38 \times 10^{-3}$ & $2.92 \times 10^{1}$ \\
$2.18 \times 10^{-5}$ & $2.85 \times 10^{-3}$ & $3.52 \times 10^{1}$ \\
\hline$k_{2}=(1.27 \pm 0.04) \times 10^{4} \mathrm{M}^{-1} \mathrm{~s}^{-1}$ &
\end{tabular}

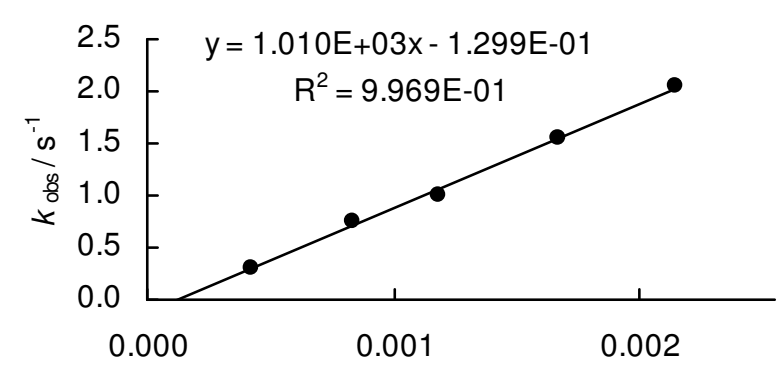

[2f] / M
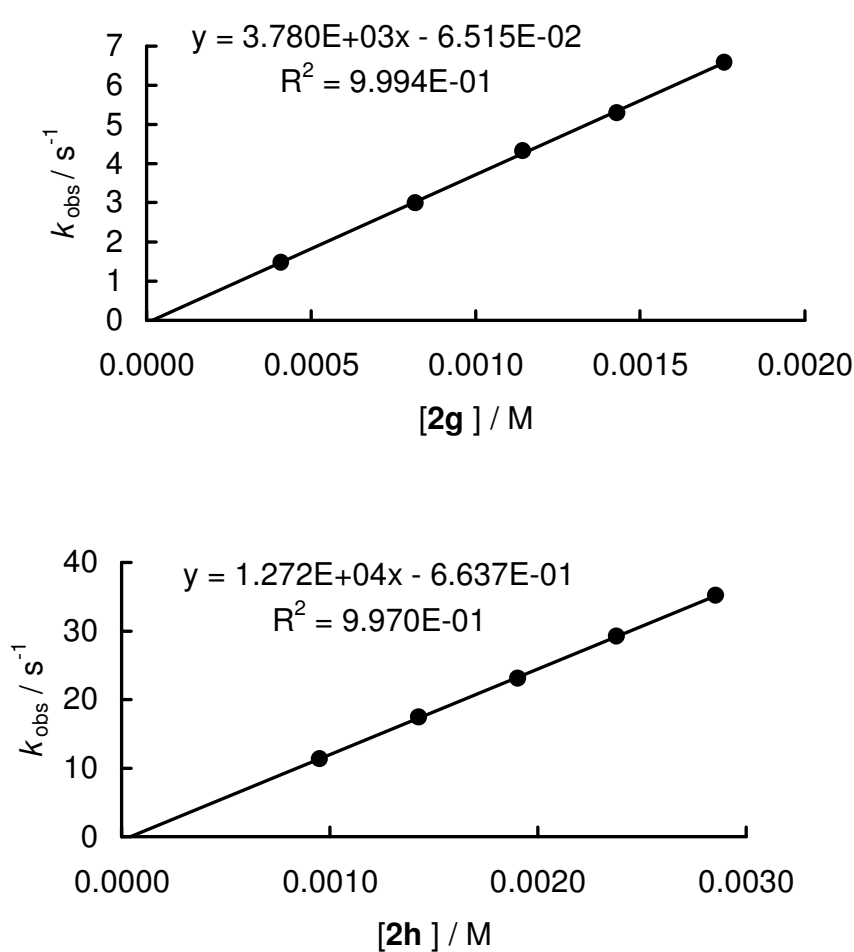
reaction of $\mathbf{1 a}$ with $\mathbf{2} \mathbf{i}$

(DMSO, $20^{\circ} \mathrm{C}$, stopped flow, $500 \mathrm{~nm}$ )

\begin{tabular}{ccc}
\hline$[1 \mathrm{a}] / \mathrm{M}$ & {$[\mathbf{2 i}] / \mathrm{M}$} & $k_{\text {obs }} / \mathrm{s}^{-1}$ \\
\hline $2.90 \times 10^{-5}$ & $5.87 \times 10^{-4}$ & 4.36 \\
$2.90 \times 10^{-5}$ & $7.83 \times 10^{-4}$ & 5.75 \\
$2.90 \times 10^{-5}$ & $9.78 \times 10^{-4}$ & 7.35 \\
$2.90 \times 10^{-5}$ & $1.17 \times 10^{-3}$ & 8.82 \\
$2.90 \times 10^{-5}$ & $1.37 \times 10^{-3}$ & $1.00 \times 10^{1}$ \\
\hline$k_{2}=(7.34 \pm 0.20) \times 10^{3} \mathrm{M}^{-1} \mathrm{~s}^{-1}$ &
\end{tabular}

reaction of $\mathbf{1 a}$ with $\mathbf{2 k}$

(DMSO, $20^{\circ} \mathrm{C}$, stopped flow, $500 \mathrm{~nm}$ )

\begin{tabular}{ccc}
\hline$[1 \mathrm{a}] / \mathrm{M}$ & {$[\mathbf{2 k}] / \mathrm{M}$} & $k_{\mathrm{obs}} / \mathrm{s}^{-1}$ \\
\hline $2.63 \times 10^{-5}$ & $5.26 \times 10^{-4}$ & 3.84 \\
$2.63 \times 10^{-5}$ & $6.48 \times 10^{-4}$ & 4.87 \\
$2.63 \times 10^{-5}$ & $7.89 \times 10^{-4}$ & 5.90 \\
$2.63 \times 10^{-5}$ & $9.11 \times 10^{-4}$ & 6.81 \\
\hline$k_{2}=(7.66 \pm 0.17) \times 10^{3} \mathrm{M}^{-1} \mathrm{~s}^{-1}$ &
\end{tabular}

reaction of $1 \mathbf{a}$ with $\mathbf{2}$

(DMSO, $20^{\circ} \mathrm{C}$, stopped flow, $500 \mathrm{~nm}$ )

\begin{tabular}{ccc}
\hline$[1 \mathrm{a}] / \mathrm{M}$ & {$[2 \mathrm{I}] / \mathrm{M}$} & $k_{\mathrm{obs}} / \mathrm{s}^{-1}$ \\
\hline $1.75 \times 10^{-5}$ & $4.76 \times 10^{-4}$ & 4.65 \\
$1.75 \times 10^{-5}$ & $9.52 \times 10^{-4}$ & $1.16 \times 10^{1}$ \\
$1.75 \times 10^{-5}$ & $1.90 \times 10^{-3}$ & $2.65 \times 10^{1}$ \\
\hline$k_{2}=(1.54 \pm 0.03) \times 10^{4} \mathrm{M}^{-1} \mathrm{~s}^{-1}$ &
\end{tabular}

reaction of $1 \mathrm{a}$ with $\mathbf{2 m}$

(DMSO, $20^{\circ} \mathrm{C}$, stopped flow, $500 \mathrm{~nm}$ )

\begin{tabular}{ccc}
\hline$[1 \mathrm{a}] / \mathrm{M}$ & {$[2 \mathrm{~m}] / \mathrm{M}$} & $k_{\mathrm{obs}} / \mathrm{s}^{-1}$ \\
\hline $2.98 \times 10^{-5}$ & $5.24 \times 10^{-4}$ & 9.81 \\
$2.98 \times 10^{-5}$ & $1.05 \times 10^{-3}$ & $2.46 \times 10^{1}$ \\
$2.98 \times 10^{-5}$ & $1.57 \times 10^{-3}$ & $4.07 \times 10^{1}$ \\
$2.98 \times 10^{-5}$ & $2.09 \times 10^{-3}$ & $5.62 \times 10^{1}$ \\
\hline$k_{2}=(2.98 \pm 0.04) \times 10^{4} \mathrm{M}^{-1} \mathrm{~s}^{-1}$ &
\end{tabular}

$k_{2}=(2.98 \pm 0.04) \times 10^{4} \mathrm{M}^{-1} \mathrm{~s}^{-1}$

\section{reactions of electrophile $1 \mathrm{~b}$}

reaction of $\mathbf{1 b}$ with $\mathbf{2 b}$

(DMSO, $20^{\circ} \mathrm{C}$, stopped flow, $487 \mathrm{~nm}$ )

\begin{tabular}{ccc}
\hline$[\mathbf{1 b}] / \mathrm{M}$ & {$[\mathbf{2 b}] / \mathrm{M}$} & $k_{\mathrm{obs}} / \mathrm{s}^{-1}$ \\
\hline $3.80 \times 10^{-5}$ & $7.78 \times 10^{-4}$ & $8.24 \times 10^{-1}$ \\
$3.80 \times 10^{-5}$ & $1.56 \times 10^{-3}$ & 1.74 \\
$3.80 \times 10^{-5}$ & $2.22 \times 10^{-3}$ & 2.50 \\
$3.80 \times 10^{-5}$ & $3.00 \times 10^{-3}$ & 3.23 \\
$3.80 \times 10^{-5}$ & $3.89 \times 10^{-3}$ & 4.07 \\
\hline$k_{2}=(1.04 \pm 0.03) \times 10^{3} \mathrm{M}^{-1} \mathrm{~s}^{-1}$ &
\end{tabular}
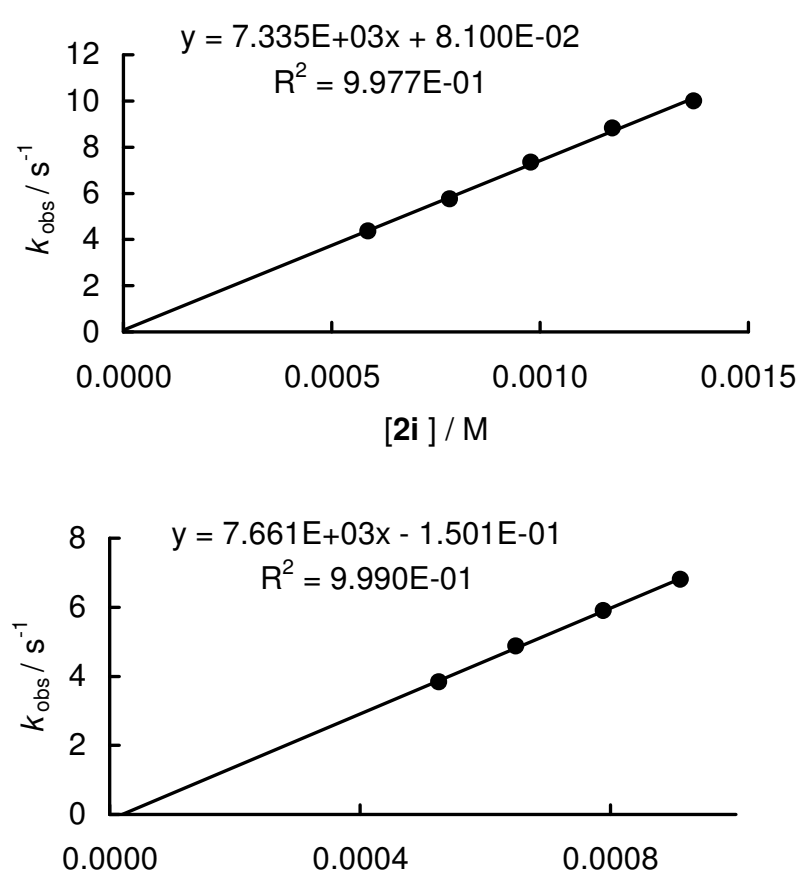

[2k ] / M

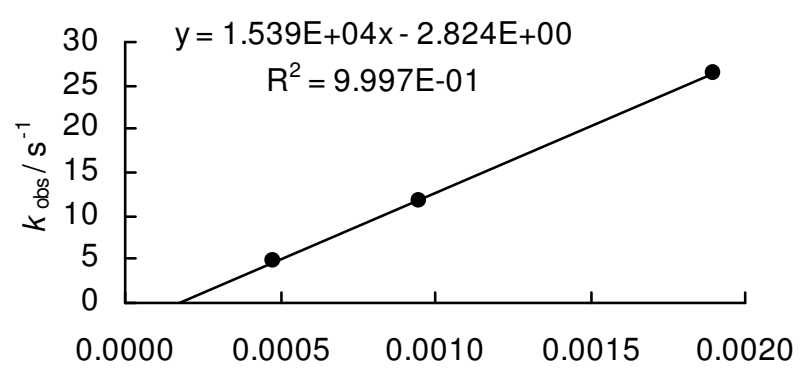

[2I ] / M
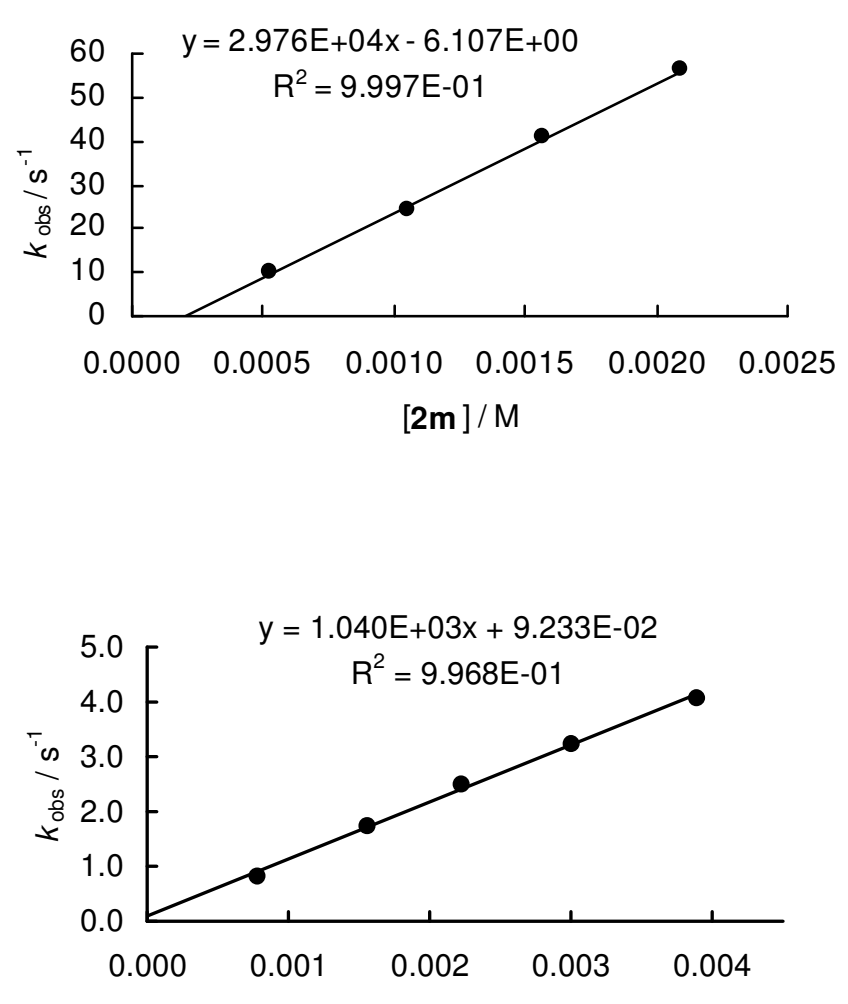

[2b] / M 
reaction of $\mathbf{1 b}$ with $2 \mathrm{c}$

(DMSO, $20{ }^{\circ} \mathrm{C}$, stopped flow, $480 \mathrm{~nm}$ )

\begin{tabular}{ccc}
\hline$[1 \mathrm{~b}] / \mathrm{M}$ & {$[\mathbf{2 c}] / \mathrm{M}$} & $k_{\text {obs }} / \mathrm{s}^{-1}$ \\
\hline $2.09 \times 10^{-5}$ & $4.09 \times 10^{-4}$ & $9.14 \times 10^{-2}$ \\
$2.09 \times 10^{-5}$ & $8.18 \times 10^{-4}$ & $1.82 \times 10^{-1}$ \\
$2.09 \times 10^{-5}$ & $9.20 \times 10^{-4}$ & $2.03 \times 10^{-1}$ \\
$2.09 \times 10^{-5}$ & $1.23 \times 10^{-3}$ & $2.74 \times 10^{-1}$ \\
$2.09 \times 10^{-5}$ & $1.64 \times 10^{-3}$ & $3.70 \times 10^{-1}$ \\
\hline$k_{2}=(2.18 \pm 0.01) \times 10^{2} \mathrm{M}^{-1} \mathrm{~s}^{-1}$ &
\end{tabular}

reaction of $1 \mathbf{b}$ with $2 \mathrm{e}$

(DMSO, $20^{\circ} \mathrm{C}$, stopped flow, $487 \mathrm{~nm}$ )

\begin{tabular}{ccc}
\hline$[\mathbf{1 b}] / \mathrm{M}$ & {$[\mathbf{2 e}] / \mathrm{M}$} & $k_{\mathrm{obs}} / \mathrm{s}^{-1}$ \\
\hline $2.09 \times 10^{-5}$ & $4.11 \times 10^{-4}$ & 1.51 \\
$2.09 \times 10^{-5}$ & $8.22 \times 10^{-4}$ & 3.05 \\
$2.09 \times 10^{-5}$ & $1.28 \times 10^{-3}$ & 4.79 \\
$2.09 \times 10^{-5}$ & $1.67 \times 10^{-3}$ & 6.01 \\
$2.09 \times 10^{-5}$ & $2.06 \times 10^{-3}$ & 7.75 \\
\hline$k_{2}=(3.72 \pm 0.10) \times 10^{3} \mathrm{M}^{-1} \mathrm{~s}^{-1}$ &
\end{tabular}

reaction of $\mathbf{1 b}$ with $\mathbf{2 f}$

DMSO, $20^{\circ} \mathrm{C}$, stopped flow, $480 \mathrm{~nm}$ )

\begin{tabular}{ccc}
\hline$[\mathbf{1 b}] / \mathrm{M}$ & {$[\mathbf{2 f}] / \mathrm{M}$} & $k_{\text {obs }} / \mathrm{s}^{-1}$ \\
\hline $2.08 \times 10^{-5}$ & $4.18 \times 10^{-4}$ & 2.01 \\
$2.08 \times 10^{-5}$ & $8.37 \times 10^{-4}$ & 4.58 \\
$2.08 \times 10^{-5}$ & $1.19 \times 10^{-3}$ & 6.61 \\
$2.08 \times 10^{-5}$ & $1.67 \times 10^{-3}$ & 9.14 \\
$2.08 \times 10^{-5}$ & $2.15 \times 10^{-3}$ & $1.20 \times 10^{1}$ \\
\hline$k_{2}=(5.71 \pm 0.08) \times 10^{3} \mathrm{M}^{-1} \mathrm{~s}^{-1}$ &
\end{tabular}

reaction of $\mathbf{1 b}$ with $\mathbf{2 g}$

(DMSO, $20^{\circ} \mathrm{C}$, stopped flow, $500 \mathrm{~nm}$ )

\begin{tabular}{ccc}
\hline$[\mathbf{1 b}] / \mathrm{M}$ & {$[\mathbf{2 g}] / \mathrm{M}$} & $k_{\text {obs }} / \mathrm{s}^{-1}$ \\
\hline $2.85 \times 10^{-5}$ & $4.08 \times 10^{-4}$ & 8.47 \\
$2.85 \times 10^{-5}$ & $8.17 \times 10^{-4}$ & $1.77 \times 10^{1}$ \\
$2.85 \times 10^{-5}$ & $1.14 \times 10^{-3}$ & $2.41 \times 10^{1}$ \\
$2.85 \times 10^{-5}$ & $1.43 \times 10^{-3}$ & $2.97 \times 10^{1}$ \\
$2.85 \times 10^{-5}$ & $1.76 \times 10^{-3}$ & $3.59 \times 10^{1}$ \\
\hline$k_{2}=(2.03 \pm 0.04) \times 10^{4} \mathrm{M}^{-1} \mathrm{~s}^{-1}$ &
\end{tabular}

reaction of $\mathbf{1 b}$ with $\mathbf{2 h}$

(DMSO, $20^{\circ} \mathrm{C}$, stopped flow, $487 \mathrm{~nm}$ )

\begin{tabular}{ccc}
\hline$[\mathbf{1 b}] / \mathrm{M}$ & {$[\mathbf{2 h}] / \mathrm{M}$} & $k_{\text {obs }} / \mathrm{s}^{-1}$ \\
\hline $3.80 \times 10^{-5}$ & $9.51 \times 10^{-4}$ & $5.65 \times 10^{1}$ \\
$3.80 \times 10^{-5}$ & $1.43 \times 10^{-3}$ & $8.68 \times 10^{1}$ \\
$3.80 \times 10^{-5}$ & $1.90 \times 10^{-3}$ & $1.14 \times 10^{2}$ \\
$3.80 \times 10^{-5}$ & $2.38 \times 10^{-3}$ & $1.39 \times 10^{2}$ \\
$3.80 \times 10^{-5}$ & $2.85 \times 10^{-3}$ & $1.70 \times 10^{2}$ \\
\hline \multicolumn{2}{l}{$k_{2}=(5.88 \pm 0.12) \times 10^{4} \mathrm{M}^{-1} \mathrm{~s}^{-1}$} &
\end{tabular}
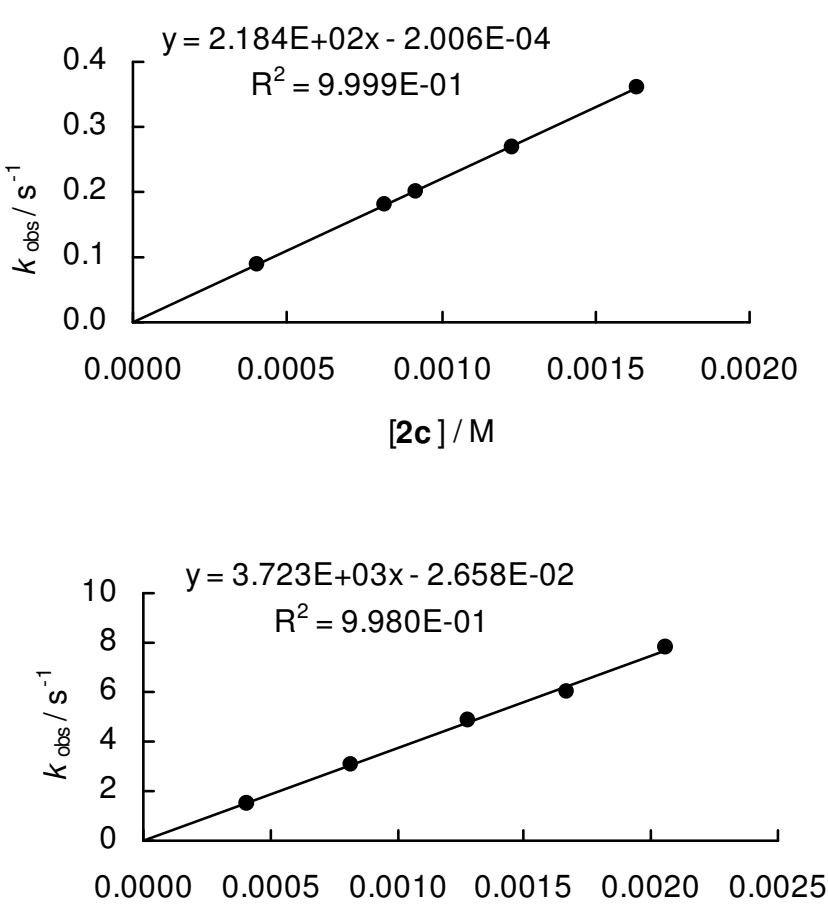

$[2 \mathrm{e}] / \mathrm{M}$
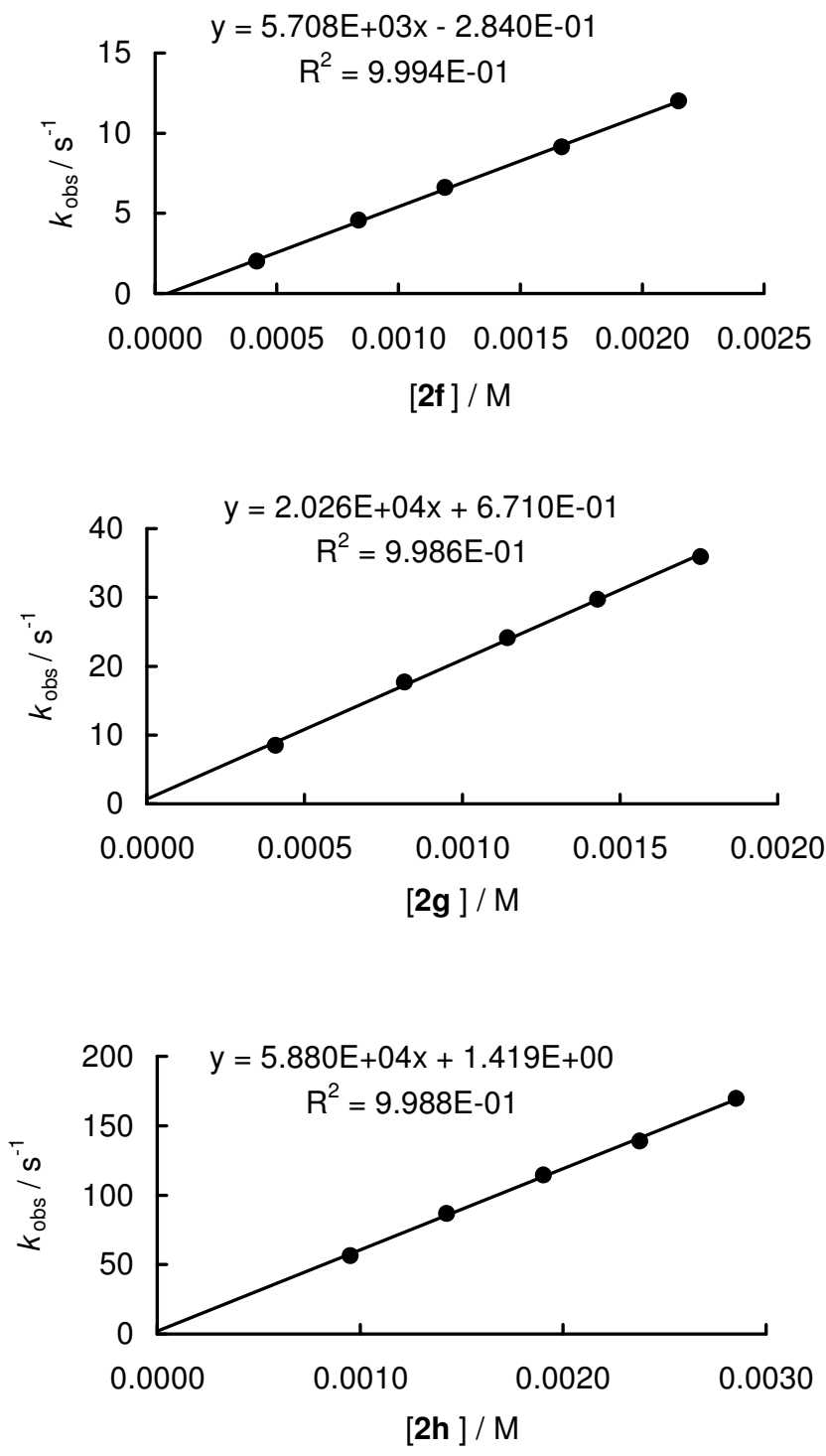
reaction of $\mathbf{1} \mathbf{b}$ with $\mathbf{2} \mathbf{i}$

(DMSO, $20^{\circ} \mathrm{C}$, stopped flow, $500 \mathrm{~nm}$ )

\begin{tabular}{ccc}
\hline$[\mathbf{1 b}] / \mathrm{M}$ & {$[\mathbf{2 i}] / \mathrm{M}$} & $k_{\mathrm{obs}} / \mathrm{s}^{-1}$ \\
\hline $4.00 \times 10^{-5}$ & $5.87 \times 10^{-4}$ & $2.31 \times 10^{1}$ \\
$4.00 \times 10^{-5}$ & $7.83 \times 10^{-4}$ & $3.11 \times 10^{1}$ \\
$4.00 \times 10^{-5}$ & $9.78 \times 10^{-4}$ & $3.88 \times 10^{1}$ \\
$4.00 \times 10^{-5}$ & $1.17 \times 10^{-3}$ & $4.73 \times 10^{1}$ \\
$4.00 \times 10^{-5}$ & $1.37 \times 10^{-3}$ & $5.41 \times 10^{1}$ \\
\hline$k_{2}=(4.00 \pm 0.07) \times 10^{4} \mathrm{M}^{-1} \mathrm{~s}^{-1}$ &
\end{tabular}

reaction of $\mathbf{1 b}$ with $\mathbf{2 k}$

(DMSO, $20^{\circ} \mathrm{C}$, stopped flow, $500 \mathrm{~nm}$ )

reaction of $\mathbf{1 b}$ with propyl amine

(DMSO, $20{ }^{\circ} \mathrm{C}$, stopped flow, $480 \mathrm{~nm}$ )

\begin{tabular}{ccc}
\hline$[1 \mathrm{~b}] / \mathrm{M}$ & [amine] $/ \mathrm{M}$ & $k_{\text {obs }} / \mathrm{s}^{-1}$ \\
\hline $4.57 \times 10^{-5}$ & $6.00 \times 10^{-3}$ & $1.24 \times 10^{1}$ \\
$4.57 \times 10^{-5}$ & $9.01 \times 10^{-3}$ & $1.88 \times 10^{1}$ \\
$4.57 \times 10^{-5}$ & $1.20 \times 10^{-2}$ & $2.51 \times 10^{1}$ \\
$4.57 \times 10^{-5}$ & $1.50 \times 10^{-2}$ & $3.21 \times 10^{1}$ \\
$4.57 \times 10^{-5}$ & $1.80 \times 10^{-2}$ & $3.76 \times 10^{1}$ \\
\hline$k_{2}=(2.12 \pm 0.04) \times 10^{3} \mathrm{M}^{-1} \mathrm{~s}^{-1}$ &
\end{tabular}

reaction of $\mathbf{1 b}$ with morpholine

(DMSO, $20^{\circ} \mathrm{C}$, stopped flow, $480 \mathrm{~nm}$ )

\begin{tabular}{ccc}
\hline$[\mathbf{1 b}] / \mathrm{M}$ & {$[$ amine] $/ \mathrm{M}$} & $k_{\mathrm{obs}} / \mathrm{s}^{-1}$ \\
\hline $2.51 \times 10^{-5}$ & $2.50 \times 10^{-3}$ & $1.43 \times 10^{2}$ \\
$2.51 \times 10^{-5}$ & $4.49 \times 10^{-3}$ & $1.78 \times 10^{2}$ \\
$2.51 \times 10^{-5}$ & $6.56 \times 10^{-3}$ & $2.20 \times 10^{2}$ \\
$2.51 \times 10^{-5}$ & $8.55 \times 10^{-3}$ & $2.58 \times 10^{2}$ \\
$2.51 \times 10^{-5}$ & $1.05 \times 10^{-2}$ & $3.05 \times 10^{2}$ \\
\hline$k_{2}=(2.01 \pm 0.06) \times 10^{4} \mathrm{M}^{-1} \mathrm{~s}^{-1}$ &
\end{tabular}

\begin{tabular}{ccc}
\hline$[\mathbf{1 b}] / \mathrm{M}$ & {$[\mathbf{2 k}] / \mathrm{M}$} & $k_{\mathrm{obs}} / \mathrm{s}^{-1}$ \\
\hline $3.03 \times 10^{-5}$ & $4.38 \times 10^{-4}$ & $1.65 \times 10^{1}$ \\
$3.03 \times 10^{-5}$ & $6.48 \times 10^{-4}$ & $2.41 \times 10^{1}$ \\
$3.03 \times 10^{-5}$ & $7.89 \times 10^{-4}$ & $2.87 \times 10^{1}$ \\
$3.03 \times 10^{-5}$ & $9.11 \times 10^{-4}$ & $3.31 \times 10^{1}$ \\
\hline \multicolumn{2}{l}{$k_{2}=(3.49 \pm 0.05) \times 10^{4} \mathrm{M}^{-1} \mathrm{~s}^{-1}$}
\end{tabular}
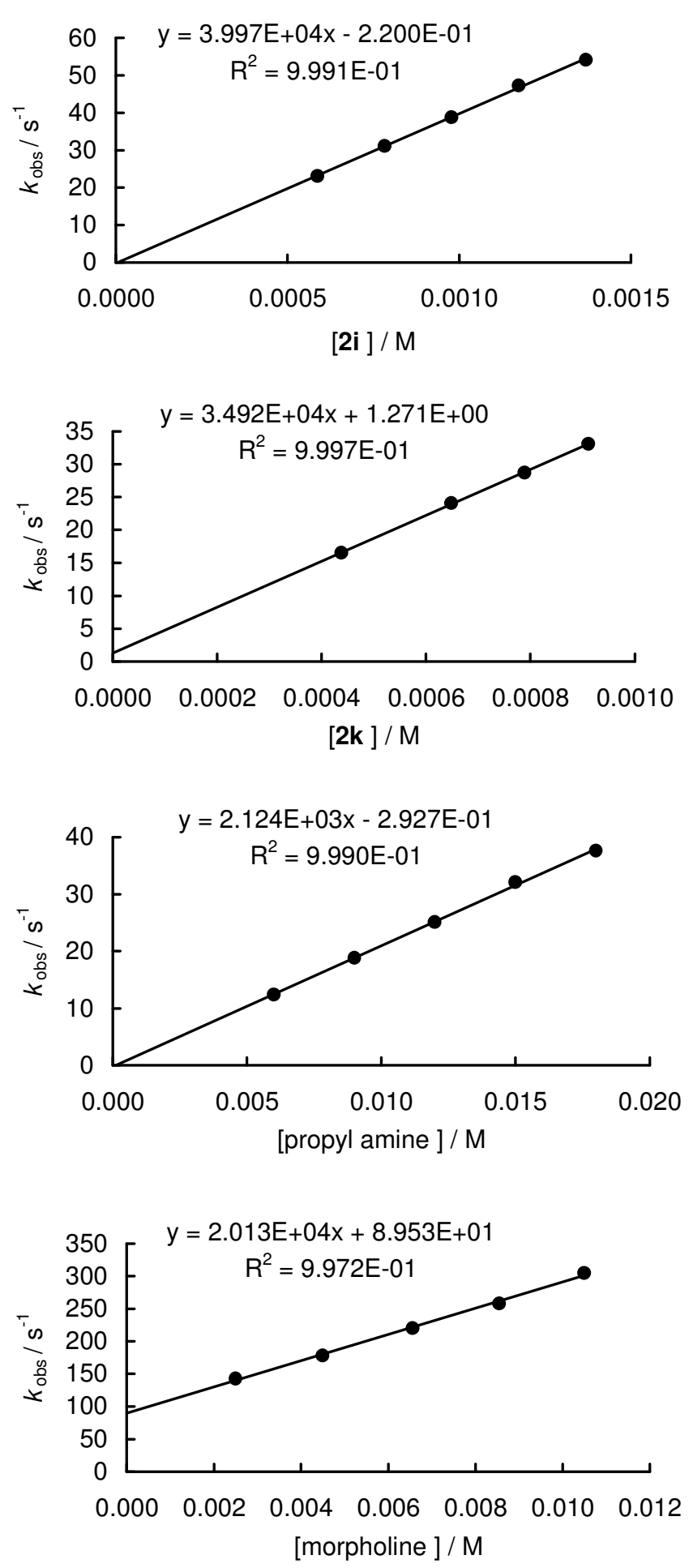

\section{reactions of electrophile 1c}

reaction of $1 \mathbf{c}$ with $\mathbf{2 a}$

(DMSO, $20^{\circ} \mathrm{C}$, stopped flow, $364 \mathrm{~nm}$ )

\begin{tabular}{ccc}
\hline$[1 \mathrm{c}] / \mathrm{M}$ & {$[\mathbf{2 a}] / \mathrm{M}$} & $k_{\text {obs }} / \mathrm{s}^{-1}$ \\
\hline $1.82 \times 10^{-5}$ & $2.96 \times 10^{-4}$ & $5.37 \times 10^{-1}$ \\
$1.82 \times 10^{-5}$ & $5.93 \times 10^{-4}$ & 1.05 \\
$1.82 \times 10^{-5}$ & $8.89 \times 10^{-4}$ & 1.41 \\
$1.82 \times 10^{-5}$ & $1.19 \times 10^{-3}$ & 1.82 \\
\hline$k_{2}=(1.42 \pm 0.07) \times 10^{3} \mathrm{M}^{-1} \mathrm{~s}^{-1}$ &
\end{tabular}

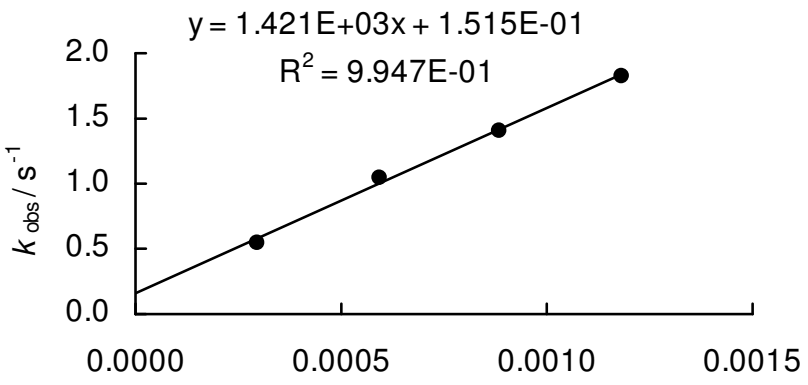

[2a ]/M 
reaction of $\mathbf{1 c}$ with $\mathbf{2 b}$

(DMSO, $20{ }^{\circ} \mathrm{C}$, stopped flow, $364 \mathrm{~nm}$ )

\begin{tabular}{ccc}
\hline$[\mathbf{1 c}] / \mathrm{M}$ & {$[\mathbf{2 b}] / \mathrm{M}$} & $k_{\mathrm{obs}} / \mathrm{s}^{-1}$ \\
\hline $1.82 \times 10^{-5}$ & $2.78 \times 10^{-4}$ & $1.27 \times 10^{1}$ \\
$1.82 \times 10^{-5}$ & $5.55 \times 10^{-4}$ & $2.64 \times 10^{1}$ \\
$1.82 \times 10^{-5}$ & $8.33 \times 10^{-4}$ & $3.99 \times 10^{1}$ \\
$1.82 \times 10^{-5}$ & $1.11 \times 10^{-3}$ & $5.43 \times 10^{1}$ \\
$1.82 \times 10^{-5}$ & $1.39 \times 10^{-3}$ & $6.59 \times 10^{1}$ \\
\hline$k_{2}=(4.83 \pm 0.09) \times 10^{4} \mathrm{M}^{-1} \mathrm{~s}^{-1}$ &
\end{tabular}

reaction of $1 \mathrm{c}$ with $2 \mathrm{e}$

(DMSO, $20{ }^{\circ} \mathrm{C}$, stopped flow, $364 \mathrm{~nm}$ )

\begin{tabular}{ccc}
\hline$[\mathbf{1 c}] / \mathrm{M}$ & {$[\mathbf{2 e}] / \mathrm{M}$} & $k_{\mathrm{obs}} / \mathrm{s}^{-1}$ \\
\hline $1.82 \times 10^{-5}$ & $4.12 \times 10^{-4}$ & $5.95 \times 10^{1}$ \\
$1.82 \times 10^{-5}$ & $8.25 \times 10^{-4}$ & $1.23 \times 10^{2}$ \\
$1.82 \times 10^{-5}$ & $1.24 \times 10^{-3}$ & $1.75 \times 10^{2}$ \\
$1.82 \times 10^{-5}$ & $1.65 \times 10^{-3}$ & $2.40 \times 10^{2}$ \\
$1.82 \times 10^{-5}$ & $2.05 \times 10^{-3}$ & $2.96 \times 10^{2}$ \\
\hline$k_{2}=(1.44 \pm 0.02) \times 10^{5} \mathrm{M}^{-1} \mathrm{~s}^{-1}$ &
\end{tabular}

reaction of $1 \mathrm{c}$ with $2 \mathrm{f}$

(DMSO, $20^{\circ} \mathrm{C}$, stopped flow, $400 \mathrm{~nm}$ )

\begin{tabular}{ccc}
\hline$[1 \mathrm{c}] / \mathrm{M}$ & {$[\mathbf{2 f}] / \mathrm{M}$} & $k_{\text {obs }} / \mathrm{s}^{-1}$ \\
\hline $3.64 \times 10^{-5}$ & $2.14 \times 10^{-4}$ & $3.04 \times 10^{1}$ \\
$3.64 \times 10^{-5}$ & $4.28 \times 10^{-4}$ & $7.06 \times 10^{1}$ \\
$3.64 \times 10^{-5}$ & $6.42 \times 10^{-4}$ & $1.12 \times 10^{2}$ \\
$3.64 \times 10^{-5}$ & $8.56 \times 10^{-4}$ & $1.52 \times 10^{2}$ \\
$3.64 \times 10^{-5}$ & $1.07 \times 10^{-3}$ & $2.00 \times 10^{2}$ \\
\hline$k_{2}=(1.97 \pm 0.04) \times 10^{5} \mathrm{M}^{-1} \mathrm{~s}^{-1}$ &
\end{tabular}

reaction of $\mathbf{1 c}$ with $\mathbf{2 g}$

(DMSO, $20^{\circ} \mathrm{C}$, stopped flow, $375 \mathrm{~nm}$ )

\begin{tabular}{ccc}
\multicolumn{2}{c}{ (DMSO, $20{ }^{\circ} \mathrm{C}$, stopped flow, $\left.375 \mathrm{~nm}\right)$} \\
\hline$[\mathbf{1 c}] / \mathrm{M}$ & {$[\mathbf{2 g}] / \mathrm{M}$} & $k_{\text {obs }} / \mathrm{s}^{-1}$ \\
\hline $2.81 \times 10^{-5}$ & $3.42 \times 10^{-4}$ & $3.52 \times 10^{2}$ \\
$2.81 \times 10^{-5}$ & $3.94 \times 10^{-4}$ & $4.31 \times 10^{2}$ \\
$2.81 \times 10^{-5}$ & $4.47 \times 10^{-4}$ & $4.74 \times 10^{2}$ \\
$2.81 \times 10^{-5}$ & $4.99 \times 10^{-4}$ & $5.27 \times 10^{2}$ \\
\hline$k_{2}=(1.08 \pm 0.09) \times 10^{6} \mathrm{M}^{-1} \mathrm{~s}^{-1}$ &
\end{tabular}

reaction of $1 \mathrm{c}$ with $\mathbf{2 h}$

(DMSO, $20{ }^{\circ} \mathrm{C}$, stopped flow, $375 \mathrm{~nm}$ )

\begin{tabular}{ccc}
\hline$[\mathbf{1 c}] / \mathrm{M}$ & {$[\mathbf{2 h}] / \mathrm{M}$} & $k_{\text {obs }} / \mathrm{s}^{-1}$ \\
\hline $2.92 \times 10^{-5}$ & $2.85 \times 10^{-4}$ & $5.06 \times 10^{2}$ \\
$2.92 \times 10^{-5}$ & $3.72 \times 10^{-4}$ & $7.00 \times 10^{2}$ \\
$2.92 \times 10^{-5}$ & $4.60 \times 10^{-4}$ & $8.30 \times 10^{2}$ \\
$2.92 \times 10^{-5}$ & $5.69 \times 10^{-4}$ & $1.03 \times 10^{3}$ \\
$2.92 \times 10^{-5}$ & $6.57 \times 10^{-4}$ & $1.19 \times 10^{3}$ \\
\hline$k_{2}=(1.80 \pm 0.05) \times 10^{6} \mathrm{M}^{-1} \mathrm{~s}^{-1}$ &
\end{tabular}
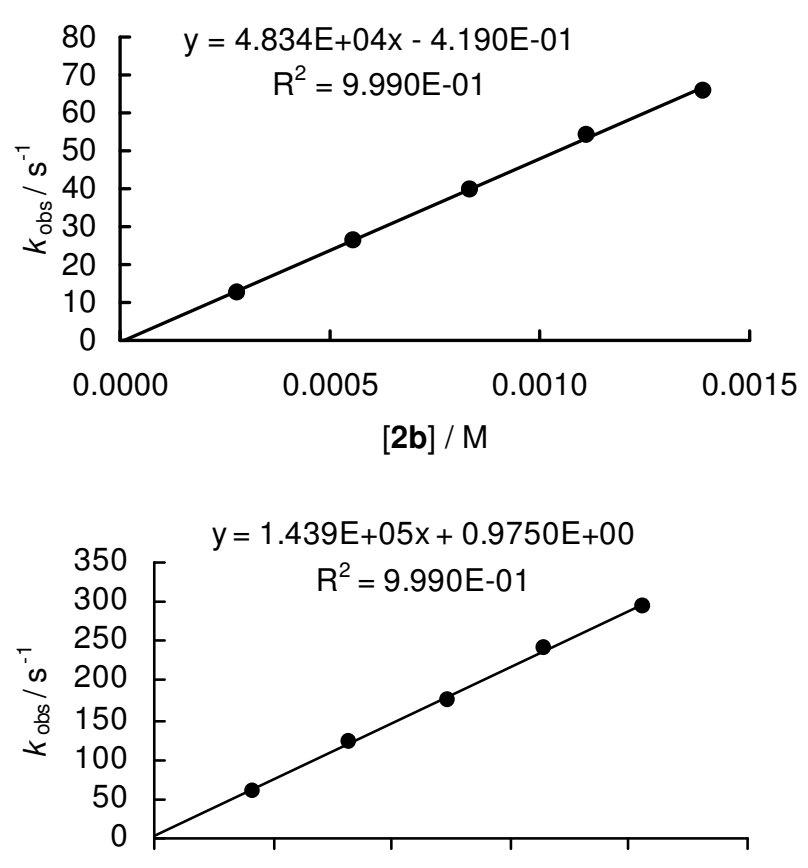

$\begin{array}{llllll}0.0000 & 0.0005 & 0.0010 & 0.0015 & 0.0020 & 0.0025\end{array}$

[2e] / M

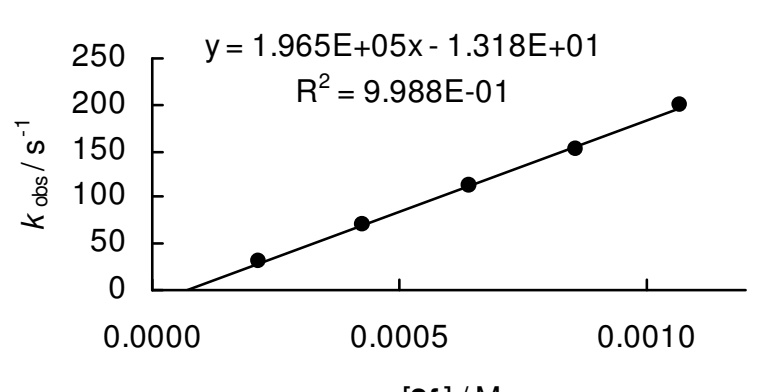

$[2 \mathrm{f}] / \mathrm{M}$

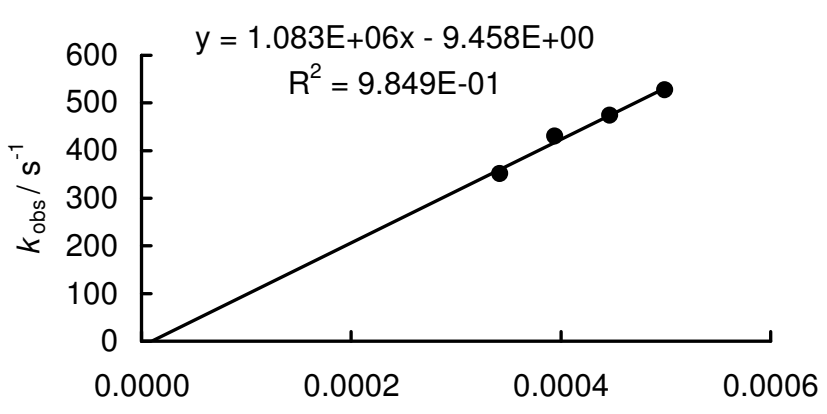

[2g] / M

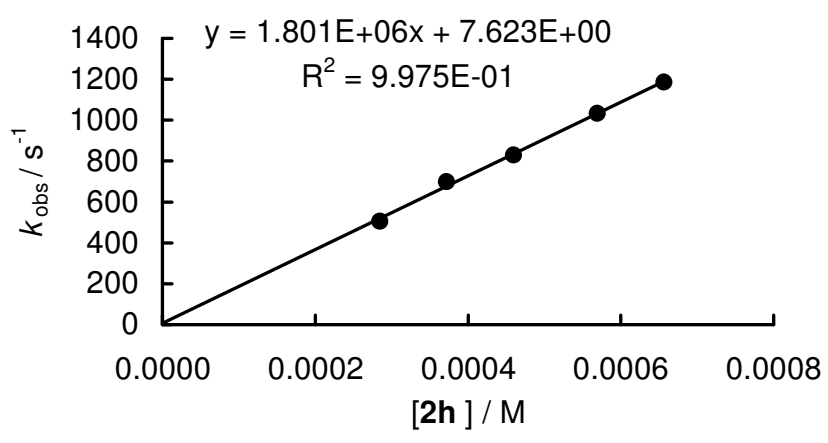


reaction of $1 \mathbf{c}$ with $\mathbf{2} \mathbf{i}$

(DMSO, $20^{\circ} \mathrm{C}$, stopped flow, $375 \mathrm{~nm}$ )

\begin{tabular}{ccc}
\hline$[1 \mathrm{c}] / \mathrm{M}$ & {$[\mathbf{2 i}] / \mathrm{M}$} & $k_{\text {obs }} / \mathrm{s}^{-1}$ \\
\hline $2.81 \times 10^{-5}$ & $2.92 \times 10^{-4}$ & $4.35 \times 10^{2}$ \\
$2.81 \times 10^{-5}$ & $3.40 \times 10^{-4}$ & $5.04 \times 10^{2}$ \\
$2.81 \times 10^{-5}$ & $3.89 \times 10^{-4}$ & $5.74 \times 10^{2}$ \\
$2.81 \times 10^{-5}$ & $4.47 \times 10^{-4}$ & $6.73 \times 10^{2}$ \\
$2.81 \times 10^{-5}$ & $5.06 \times 10^{-4}$ & $7.49 \times 10^{2}$ \\
\hline$k_{2}=(1.49 \pm 0.03) \times 10^{6} \mathrm{M}^{-1} \mathrm{~s}^{-1}$ &
\end{tabular}

reaction of $1 \mathbf{c}$ with $\mathbf{2 k}$

(DMSO, $20^{\circ} \mathrm{C}$, stopped flow, $375 \mathrm{~nm}$ )

\begin{tabular}{ccc}
\hline$[1 \mathrm{c}] / \mathrm{M}$ & {$[\mathbf{2 k}] / \mathrm{M}$} & $k_{\mathrm{obs}} / \mathrm{s}^{-1}$ \\
\hline $2.81 \times 10^{-5}$ & $2.85 \times 10^{-4}$ & $3.98 \times 10^{2}$ \\
$2.81 \times 10^{-5}$ & $3.40 \times 10^{-4}$ & $4.53 \times 10^{2}$ \\
$2.81 \times 10^{-5}$ & $3.96 \times 10^{-4}$ & $5.60 \times 10^{2}$ \\
$2.81 \times 10^{-5}$ & $4.51 \times 10^{-4}$ & $6.12 \times 10^{2}$ \\
$2.81 \times 10^{-5}$ & $5.07 \times 10^{-4}$ & $7.09 \times 10^{2}$ \\
\hline$k_{2}=(1.41 \pm 0.09) \times 10^{6} \mathrm{M}^{-1} \mathrm{~s}^{-1}$ &
\end{tabular}

reaction of $1 \mathrm{c}$ with $10 \mathrm{c}$

(DMSO, $20{ }^{\circ} \mathrm{C}$, stopped flow, $375 \mathrm{~nm}$ )

\begin{tabular}{ccc}
\hline$[1 \mathrm{c}] / \mathrm{M}$ & {$[\mathbf{1 0 c}] / \mathrm{M}$} & $k_{\mathrm{obs}} / \mathrm{s}^{-1}$ \\
\hline $1.23 \times 10^{-4}$ & $1.75 \times 10^{-2}$ & $1.66 \times 10^{-6}$ \\
$1.09 \times 10^{-4}$ & $3.26 \times 10^{-2}$ & $3.53 \times 10^{-6}$ \\
\hline$k_{2}=1.24 \times 10^{-4} \mathrm{M}^{-1} \mathrm{~s}^{-1}$
\end{tabular}

reaction of $1 \mathrm{c}$ with propyl amine

(DMSO, $20^{\circ} \mathrm{C}$, stopped flow, $380 \mathrm{~nm}$ )

\begin{tabular}{ccc}
\hline$[1 \mathrm{c}] / \mathrm{M}$ & {$[$ amine] $/ \mathrm{M}$} & $k_{\mathrm{obs}} / \mathrm{s}^{-1}$ \\
\hline $4.41 \times 10^{-5}$ & $5.77 \times 10^{-3}$ & $1.61 \times 10^{2}$ \\
$4.41 \times 10^{-5}$ & $8.84 \times 10^{-3}$ & $2.54 \times 10^{2}$ \\
$4.41 \times 10^{-5}$ & $1.19 \times 10^{-2}$ & $3.61 \times 10^{2}$ \\
$4.41 \times 10^{-5}$ & $1.41 \times 10^{-2}$ & $4.37 \times 10^{2}$ \\
$4.41 \times 10^{-5}$ & $1.77 \times 10^{-2}$ & $5.25 \times 10^{2}$ \\
\hline$k_{2}=(3.13 \pm 0.12) \times 10^{4} \mathrm{M}^{-1} \mathrm{~s}^{-1}$ &
\end{tabular}

reaction of $1 \mathrm{c}$ with morpholine

(DMSO, $20^{\circ} \mathrm{C}$, stopped flow, $380 \mathrm{~nm}$ )

\begin{tabular}{ccc}
\hline$[1 \mathrm{c}] / \mathrm{M}$ & {$[\mathrm{amine}] / \mathrm{M}$} & $k_{\text {obs }} / \mathrm{s}^{-1}$ \\
\hline $5.04 \times 10^{-5}$ & $2.60 \times 10^{-3}$ & $5.83 \times 10^{2}$ \\
$5.04 \times 10^{-5}$ & $3.75 \times 10^{-3}$ & $8.71 \times 10^{2}$ \\
$5.04 \times 10^{-5}$ & $6.34 \times 10^{-3}$ & $1.38 \times 10^{3}$ \\
$5.04 \times 10^{-5}$ & $7.21 \times 10^{-3}$ & $1.52 \times 10^{3}$ \\
\hline$k_{2}=(2.02 \pm 0.09) \times 10^{5} \mathrm{M}^{-1} \mathrm{~s}^{-1}$ &
\end{tabular}
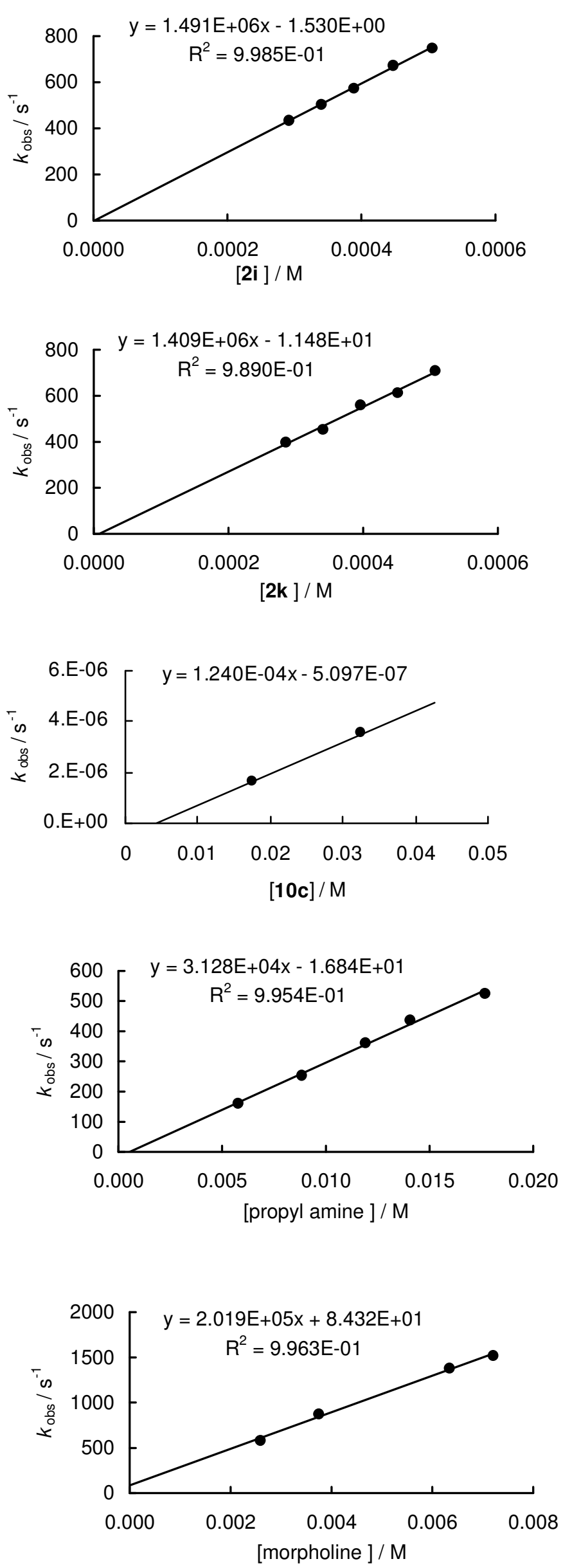


\section{reactions of electrophile $1 d$}

reaction of $\mathbf{1 d}$ with $\mathbf{2 b}$

(DMSO, $20^{\circ} \mathrm{C}$, stopped flow, $500 \mathrm{~nm}$ )

\begin{tabular}{ccc}
\hline$[\mathbf{1 d}] / \mathrm{M}$ & {$[\mathbf{2 b}] / \mathrm{M}$} & $k_{\mathrm{obs}} / \mathrm{s}^{-1}$ \\
\hline $2.30 \times 10^{-5}$ & $4.66 \times 10^{-4}$ & 1.95 \\
$2.30 \times 10^{-5}$ & $8.16 \times 10^{-4}$ & 3.46 \\
$2.30 \times 10^{-5}$ & $1.17 \times 10^{-3}$ & 4.97 \\
$2.30 \times 10^{-5}$ & $1.52 \times 10^{-3}$ & 6.54 \\
$2.30 \times 10^{-5}$ & $1.87 \times 10^{-3}$ & 8.04 \\
\hline$k_{2}=(4.36 \pm 0.02) \times 10^{3} \mathrm{M}^{-1} \mathrm{~s}^{-1}$ &
\end{tabular}

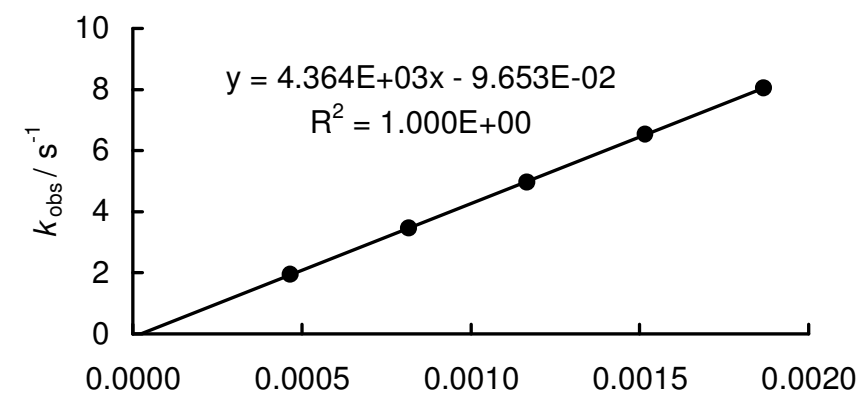

[2b ] / M

reaction of $1 \mathbf{d}$ with $2 \mathrm{e}$

(DMSO, $20^{\circ} \mathrm{C}$, stopped flow, $500 \mathrm{~nm}$ )

\begin{tabular}{ccc}
\hline$[1 \mathrm{~d}] / \mathrm{M}$ & {$[\mathbf{2 e}] / \mathrm{M}$} & $k_{\mathrm{obs}} / \mathrm{s}^{-1}$ \\
\hline $2.43 \times 10^{-5}$ & $4.16 \times 10^{-4}$ & 4.59 \\
$2.43 \times 10^{-5}$ & $8.32 \times 10^{-4}$ & 8.94 \\
$2.43 \times 10^{-5}$ & $1.25 \times 10^{-3}$ & $1.42 \times 10^{1}$ \\
$2.43 \times 10^{-5}$ & $1.66 \times 10^{-3}$ & $1.93 \times 10^{1}$ \\
$2.43 \times 10^{-5}$ & $2.08 \times 10^{-3}$ & $2.38 \times 10^{1}$ \\
\hline$k_{2}=(1.17 \pm 0.02) \times 10^{4} \mathrm{M}^{-1} \mathrm{~s}^{-1}$ &
\end{tabular}

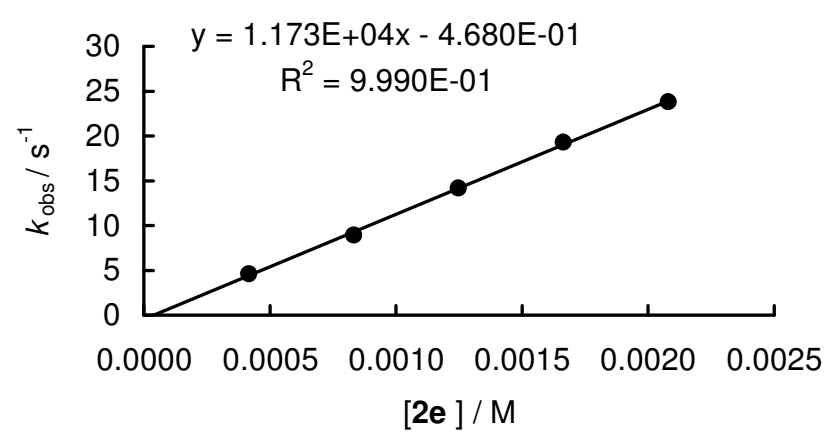

reaction of $\mathbf{1 d}$ with $\mathbf{2 g}$

(DMSO, $20^{\circ} \mathrm{C}$, stopped flow, $500 \mathrm{~nm}$ )

\begin{tabular}{ccc}
\hline$[\mathbf{1 d}] / \mathrm{M}$ & {$[\mathbf{2 g}] / \mathrm{M}$} & $k_{\mathrm{obs}} / \mathrm{s}^{-1}$ \\
\hline $4.25 \times 10^{-5}$ & $4.32 \times 10^{-4}$ & $3.21 \times 10^{1}$ \\
$4.25 \times 10^{-5}$ & $6.34 \times 10^{-4}$ & $4.81 \times 10^{1}$ \\
$4.25 \times 10^{-5}$ & $8.65 \times 10^{-4}$ & $6.45 \times 10^{1}$ \\
$4.25 \times 10^{-5}$ & $1.15 \times 10^{-3}$ & $8.65 \times 10^{1}$ \\
$4.25 \times 10^{-5}$ & $1.44 \times 10^{-3}$ & $1.07 \times 10^{2}$ \\
\hline$k_{2}=(7.41 \pm 0.07) \times 10^{4} \mathrm{M}^{-1} \mathrm{~s}^{-1}$ &
\end{tabular}

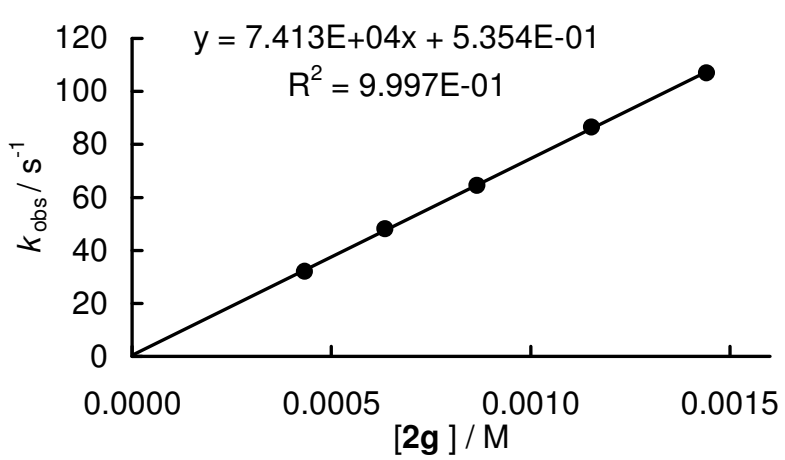

reaction of $\mathbf{1 d}$ with $\mathbf{2 h}$

(DMSO, $20^{\circ} \mathrm{C}$, stopped flow, $500 \mathrm{~nm}$ )

\begin{tabular}{ccc}
\hline$[\mathbf{1 d}] / \mathrm{M}$ & {$[\mathbf{2 h}] / \mathrm{M}$} & $k_{\mathrm{obs}} / \mathrm{s}^{-1}$ \\
\hline $5.06 \times 10^{-5}$ & $5.82 \times 10^{-4}$ & $8.64 \times 10^{1}$ \\
$5.06 \times 10^{-5}$ & $7.76 \times 10^{-4}$ & $1.16 \times 10^{2}$ \\
$5.06 \times 10^{-5}$ & $9.70 \times 10^{-4}$ & $1.44 \times 10^{2}$ \\
$5.06 \times 10^{-5}$ & $1.16 \times 10^{-3}$ & $1.83 \times 10^{2}$ \\
$5.06 \times 10^{-5}$ & $1.36 \times 10^{-3}$ & $2.12 \times 10^{2}$ \\
\hline$k_{2}=(1.64 \pm 0.05) \times 10^{5} \mathrm{M}^{-1} \mathrm{~s}^{-1}$ &
\end{tabular}

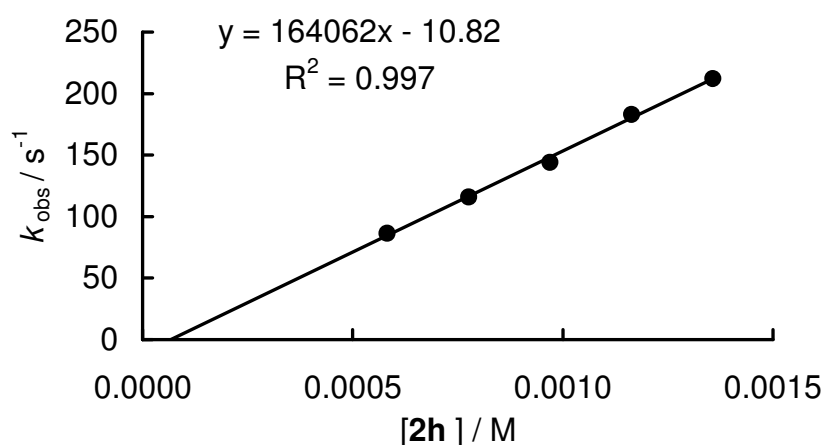

reaction of $\mathbf{1 d}$ with $\mathbf{2} \mathbf{i}$

(DMSO, $20^{\circ} \mathrm{C}$, stopped flow, $500 \mathrm{~nm}$ )

\begin{tabular}{ccc}
\hline$[1 \mathrm{~d}] / \mathrm{M}$ & {$[\mathbf{2 i}] / \mathrm{M}$} & $k_{\mathrm{obs}} / \mathrm{s}^{-1}$ \\
\hline $4.25 \times 10^{-5}$ & $5.87 \times 10^{-4}$ & $6.04 \times 10^{1}$ \\
$4.25 \times 10^{-5}$ & $8.81 \times 10^{-4}$ & $9.37 \times 10^{1}$ \\
$4.25 \times 10^{-5}$ & $1.18 \times 10^{-3}$ & $1.24 \times 10^{2}$ \\
$4.25 \times 10^{-5}$ & $1.76 \times 10^{-3}$ & $1.86 \times 10^{2}$ \\
\hline \multicolumn{2}{l}{$k_{2}=(1.06 \pm 0.01) \times 10^{5} \mathrm{M}^{-1} \mathrm{~s}^{-1}$}
\end{tabular}

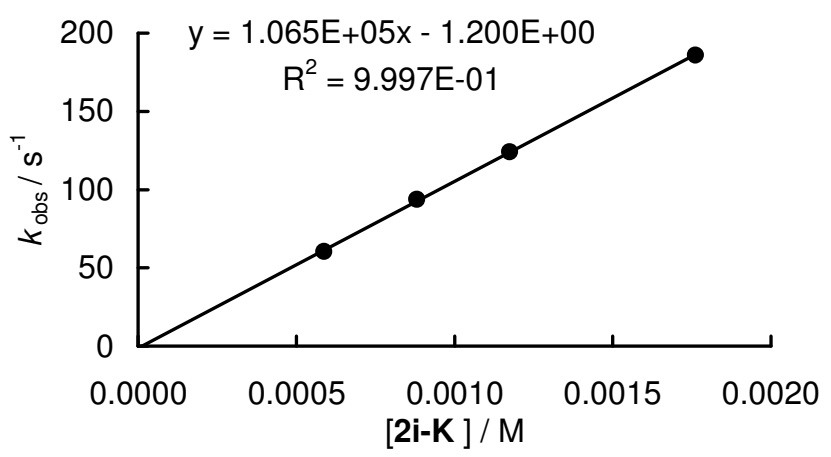


reaction of $\mathbf{1 d}$ with $\mathbf{2 k}$

(DMSO, $20^{\circ} \mathrm{C}$, stopped flow, $500 \mathrm{~nm}$ )

\begin{tabular}{ccc}
\hline$[1 \mathrm{~d}] / \mathrm{M}$ & {$[\mathbf{2 k}] / \mathrm{M}$} & $k_{\text {obs }} / \mathrm{s}^{-1}$ \\
\hline $2.59 \times 10^{-5}$ & $4.38 \times 10^{-4}$ & $4.95 \times 10^{1}$ \\
$2.59 \times 10^{-5}$ & $5.26 \times 10^{-4}$ & $5.80 \times 10^{1}$ \\
$2.59 \times 10^{-5}$ & $6.48 \times 10^{-4}$ & $7.27 \times 10^{1}$ \\
$2.59 \times 10^{-5}$ & $7.89 \times 10^{-4}$ & $8.99 \times 10^{1}$ \\
$2.59 \times 10^{-5}$ & $9.11 \times 10^{-4}$ & $1.02 \times 10^{2}$ \\
\hline$k_{2}=(1.13 \pm 0.03) \times 10^{5} \mathrm{M}^{-1} \mathrm{~s}^{-1}$ &
\end{tabular}

\section{reactions of electrophile $1 \mathrm{e}$}

reaction of $1 \mathbf{e}$ with $2 \mathrm{a}$

(DMSO, $20^{\circ} \mathrm{C}$, stopped flow, $500 \mathrm{~nm}$ )

\begin{tabular}{ccc}
\hline$[1 \mathrm{e}] / \mathrm{M}$ & {$[2 \mathrm{a}] / \mathrm{M}$} & $k_{\text {obs }} / \mathrm{s}^{-1}$ \\
\hline $3.01 \times 10^{-5}$ & $6.85 \times 10^{-4}$ & $3.26 \times 10^{-1}$ \\
$3.01 \times 10^{-5}$ & $1.20 \times 10^{-3}$ & $5.75 \times 10^{-1}$ \\
$3.01 \times 10^{-5}$ & $1.71 \times 10^{-3}$ & $8.29 \times 10^{-1}$ \\
$3.01 \times 10^{-5}$ & $2.23 \times 10^{-3}$ & 1.08 \\
$3.01 \times 10^{-5}$ & $2.74 \times 10^{-3}$ & 1.35 \\
\hline$k_{2}=(4.97 \pm 0.04) \times 10^{2} \mathrm{M}^{-1} \mathrm{~s}^{-1}$ &
\end{tabular}

reaction of $\mathbf{1 e}$ with $\mathbf{2 b}$

(DMSO, $20^{\circ} \mathrm{C}$, stopped flow, $500 \mathrm{~nm}$ )

\begin{tabular}{ccc}
\hline$[1 \mathrm{e}] / \mathrm{M}$ & {$[\mathbf{2 b}] / \mathrm{M}$} & $k_{\text {obs }} / \mathrm{s}^{-1}$ \\
\hline $2.31 \times 10^{-5}$ & $4.66 \times 10^{-4}$ & $1.42 \times 10^{1}$ \\
$2.31 \times 10^{-5}$ & $8.16 \times 10^{-4}$ & $2.71 \times 10^{1}$ \\
$2.31 \times 10^{-5}$ & $1.17 \times 10^{-3}$ & $4.05 \times 10^{1}$ \\
$2.31 \times 10^{-5}$ & $1.52 \times 10^{-3}$ & $5.31 \times 10^{1}$ \\
$2.31 \times 10^{-5}$ & $1.87 \times 10^{-3}$ & $6.62 \times 10^{1}$ \\
\hline$k_{2}=(3.72 \pm 0.02) \times 10^{4} \mathrm{M}^{-1} \mathrm{~s}^{-1}$ &
\end{tabular}

reaction of $1 \mathrm{e}$ with $\mathbf{2 e}$

(DMSO, $20^{\circ} \mathrm{C}$, stopped flow, $500 \mathrm{~nm}$ )

\begin{tabular}{ccc}
\hline$[1 \mathrm{e}] / \mathrm{M}$ & {$[\mathbf{2 e}] / \mathrm{M}$} & $k_{\mathrm{obs}} / \mathrm{s}^{-1}$ \\
\hline $2.31 \times 10^{-5}$ & $4.00 \times 10^{-4}$ & $3.88 \times 10^{1}$ \\
$2.31 \times 10^{-5}$ & $8.00 \times 10^{-4}$ & $7.86 \times 10^{1}$ \\
$2.31 \times 10^{-5}$ & $1.20 \times 10^{-3}$ & $1.19 \times 10^{2}$ \\
$2.31 \times 10^{-5}$ & $1.60 \times 10^{-3}$ & $1.62 \times 10^{2}$ \\
$2.31 \times 10^{-5}$ & $2.00 \times 10^{-3}$ & $2.04 \times 10^{2}$ \\
\hline$k_{2}=(1.03 \pm 0.01) \times 10^{5} \mathrm{M}^{-1} \mathrm{~s}^{-1}$ &
\end{tabular}
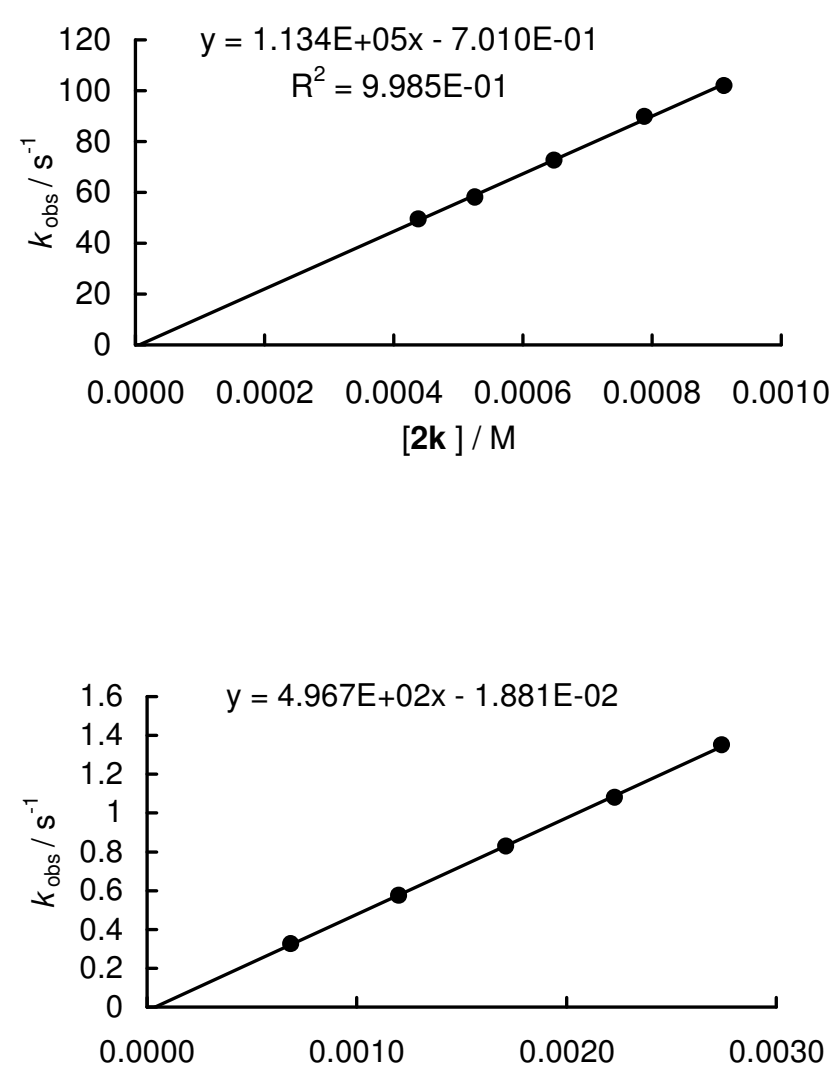

[2a ] / M
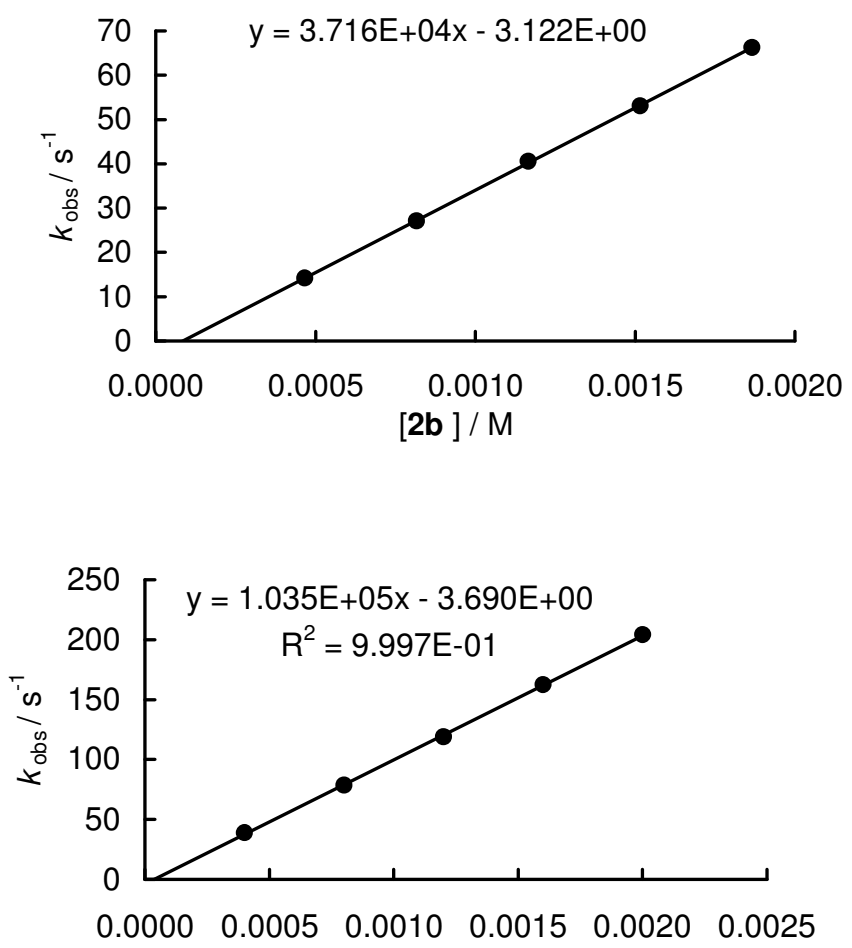

[2e] / M 
reaction of $1 \mathrm{e}$ with $\mathbf{2 g}$

(DMSO, $20^{\circ} \mathrm{C}$, stopped flow, $500 \mathrm{~nm}$ )

\begin{tabular}{ccc}
\hline$[\mathbf{1 e}] / \mathrm{M}$ & {$[\mathbf{2 g}] / \mathrm{M}$} & $k_{\text {obs }} / \mathrm{s}^{-1}$ \\
\hline $2.85 \times 10^{-5}$ & $4.32 \times 10^{-4}$ & $2.04 \times 10^{2}$ \\
$2.85 \times 10^{-5}$ & $6.34 \times 10^{-4}$ & $3.25 \times 10^{2}$ \\
$2.85 \times 10^{-5}$ & $8.65 \times 10^{-4}$ & $4.13 \times 10^{2}$ \\
$2.85 \times 10^{-5}$ & $1.15 \times 10^{-3}$ & $5.56 \times 10^{2}$ \\
$2.85 \times 10^{-5}$ & $1.44 \times 10^{-3}$ & $7.09 \times 10^{2}$ \\
\hline$k_{2}=(4.89 \pm 0.15) \times 10^{5} \mathrm{M}^{-1} \mathrm{~s}^{-1}$ &
\end{tabular}

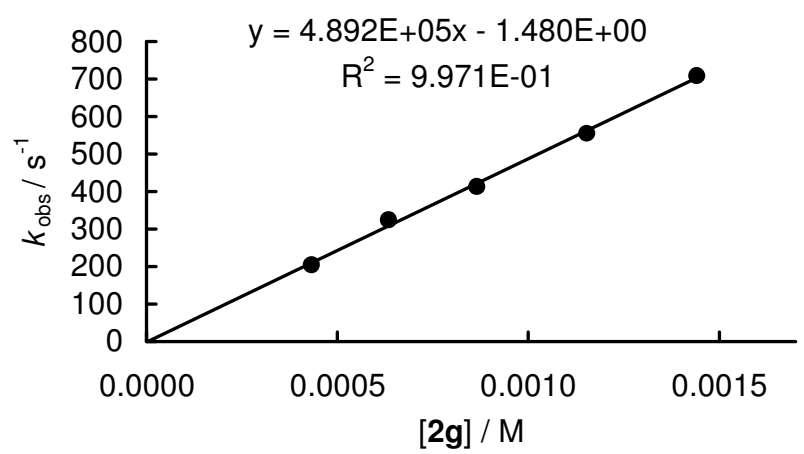

reaction of $1 \mathbf{e}$ with $2 \mathrm{i}$

(DMSO, $20^{\circ} \mathrm{C}$, stopped flow, $500 \mathrm{~nm}$ )

\begin{tabular}{ccc}
\hline$[\mathbf{1 e}] / \mathrm{M}$ & {$[\mathbf{2 i}] / \mathrm{M}$} & $k_{\mathrm{obs}} / \mathrm{s}^{-1}$ \\
\hline $2.85 \times 10^{-5}$ & $5.87 \times 10^{-4}$ & $3.63 \times 10^{2}$ \\
$2.85 \times 10^{-5}$ & $8.81 \times 10^{-4}$ & $5.78 \times 10^{2}$ \\
$2.85 \times 10^{-5}$ & $1.18 \times 10^{-3}$ & $8.19 \times 10^{2}$ \\
$2.85 \times 10^{-5}$ & $1.76 \times 10^{-3}$ & $1.23 \times 10^{3}$ \\
$2.85 \times 10^{-5}$ & $2.35 \times 10^{-3}$ & $1.60 \times 10^{3}$ \\
\hline$k_{2}=(7.05 \pm 0.18) \times 10^{5} \mathrm{M}^{-1} \mathrm{~s}^{-1}$ &
\end{tabular}

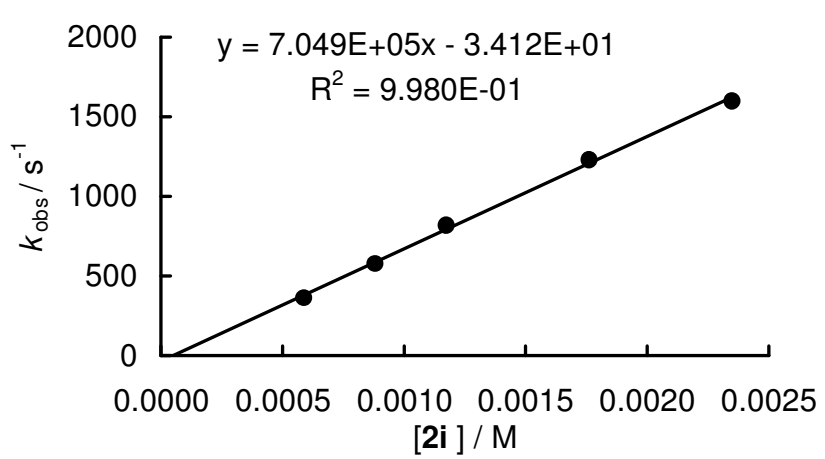

reaction of $1 \mathrm{e}$ with $\mathbf{2 k}$

(DMSO, $20^{\circ} \mathrm{C}$, stopped flow, $500 \mathrm{~nm}$ )

\begin{tabular}{ccc}
\hline$[\mathbf{1 e}] / \mathrm{M}$ & {$[\mathbf{2 k}] / \mathrm{M}$} & $k_{\mathrm{obs}} / \mathrm{s}^{-1}$ \\
\hline $3.03 \times 10^{-5}$ & $5.26 \times 10^{-4}$ & $3.39 \times 10^{2}$ \\
$3.03 \times 10^{-5}$ & $6.48 \times 10^{-4}$ & $4.15 \times 10^{2}$ \\
$3.03 \times 10^{-5}$ & $7.89 \times 10^{-4}$ & $5.00 \times 10^{2}$ \\
$3.03 \times 10^{-5}$ & $9.11 \times 10^{-4}$ & $6.01 \times 10^{2}$ \\
\hline \multicolumn{2}{l}{$k_{2}=(6.71 \pm 0.35) \times 10^{5} \mathrm{M}^{-1} \mathrm{~s}^{-1}$} &
\end{tabular}

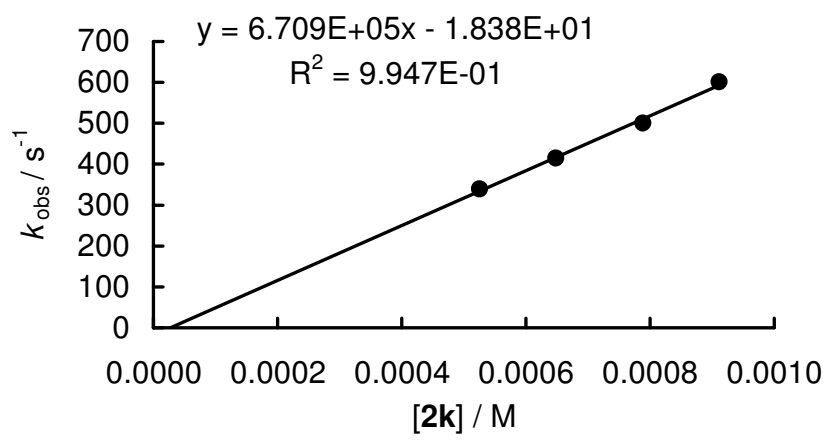

\section{NMR spectra}

Product Characterization. ${ }^{1} \mathrm{H}$ and ${ }^{13} \mathrm{C}$ NMR chemical shifts are expressed in ppm and refer to the corresponding solvents $\left(d_{6}\right.$-DMSO: $\delta_{\mathrm{H}}=2.50, \delta_{\mathrm{C}}=39.5$ and $\mathrm{CDCl}_{3}: \delta_{\mathrm{H}}=7.26$, $\delta_{\mathrm{C}}=77.2$ ). DEPT and HSQC experiments were employed to assign the signals. $d_{6}$-DMSO for NMR was distilled over $\mathrm{CaH}_{2}$ and stored under an argon atmosphere. 
nmr of products 1
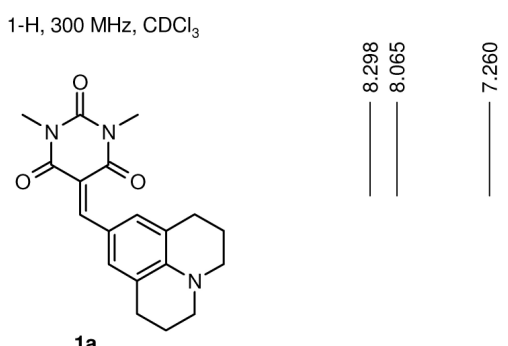

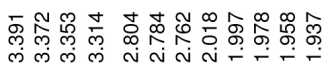
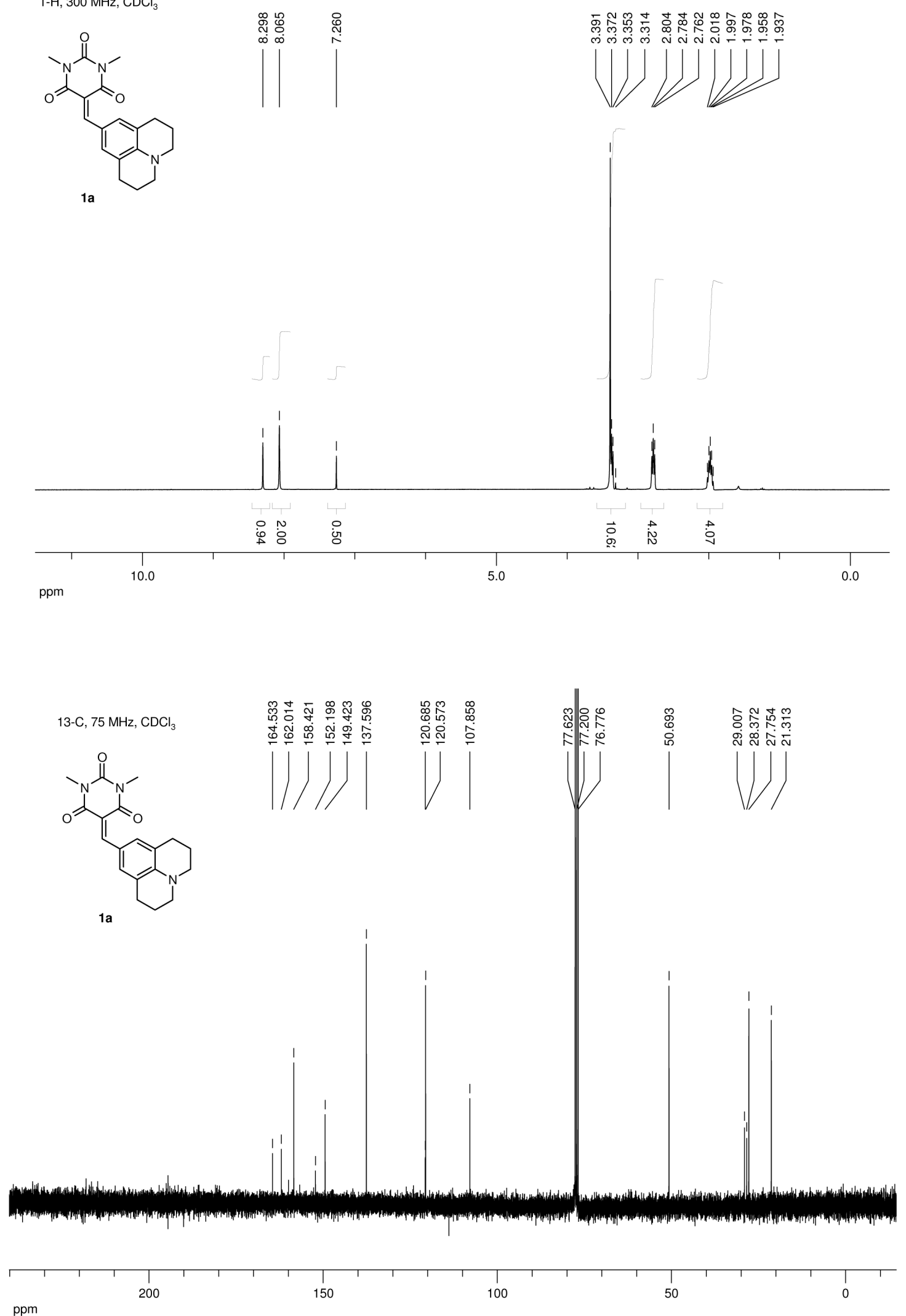

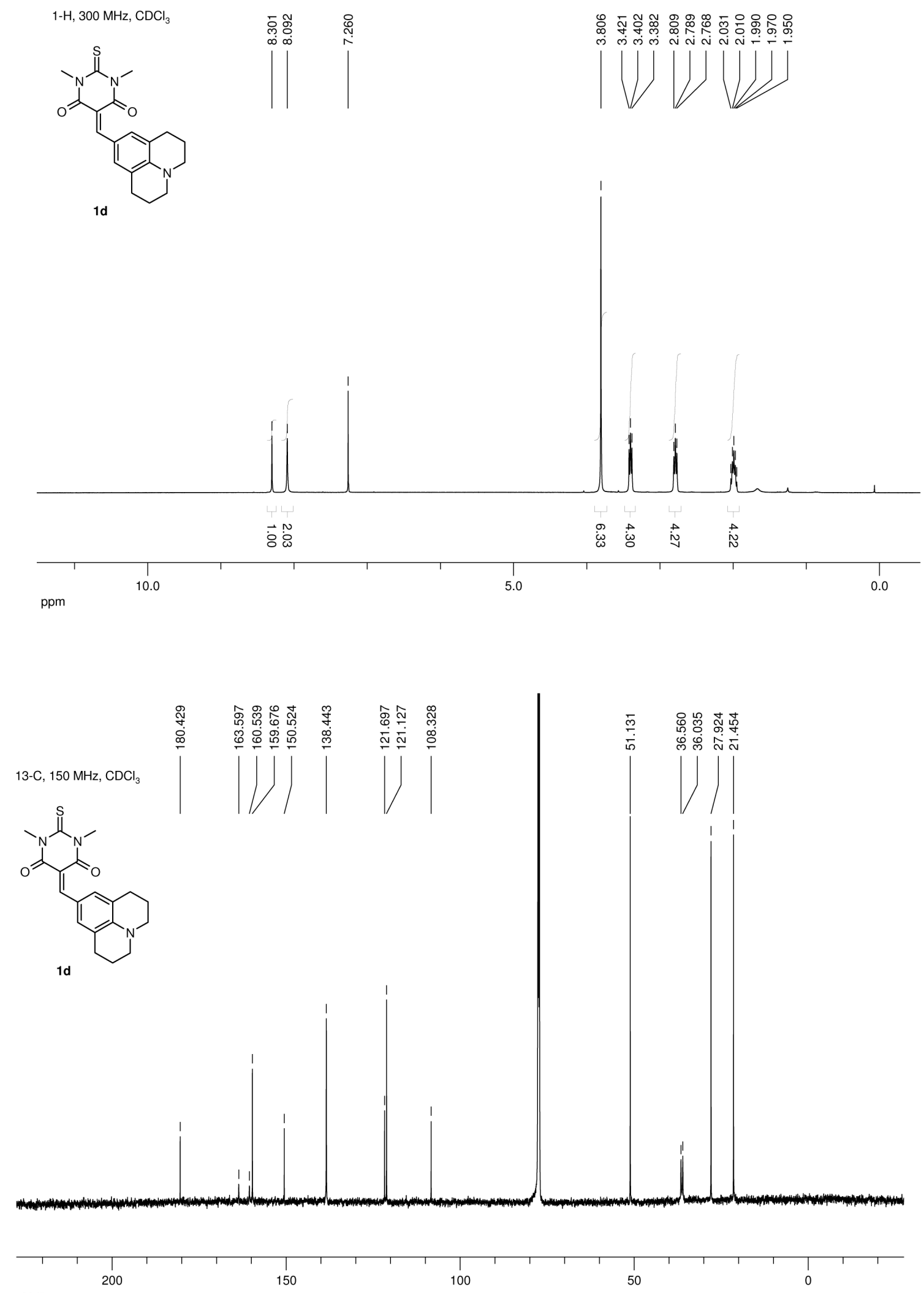

ppm 
nmr of products 3
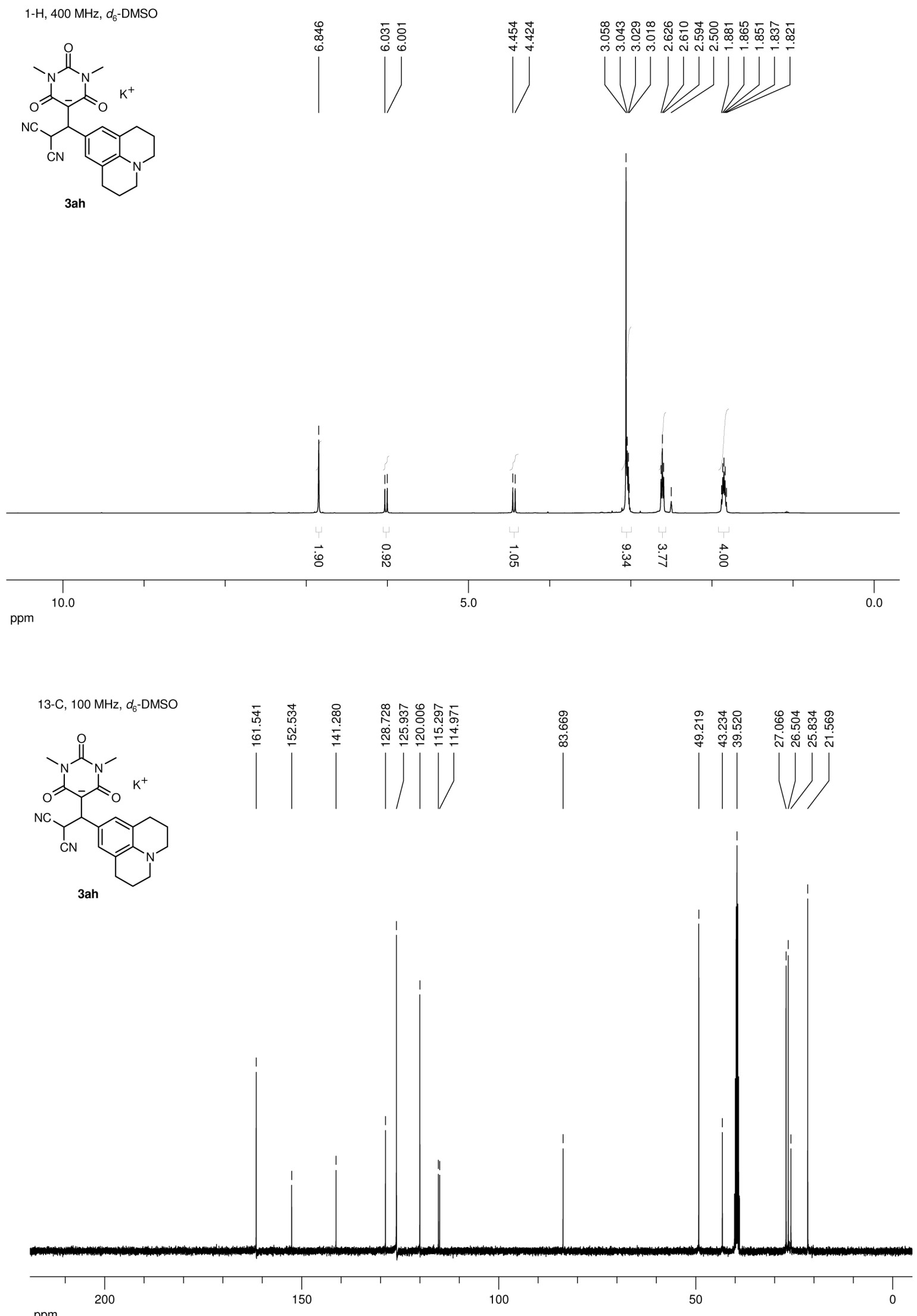
S15
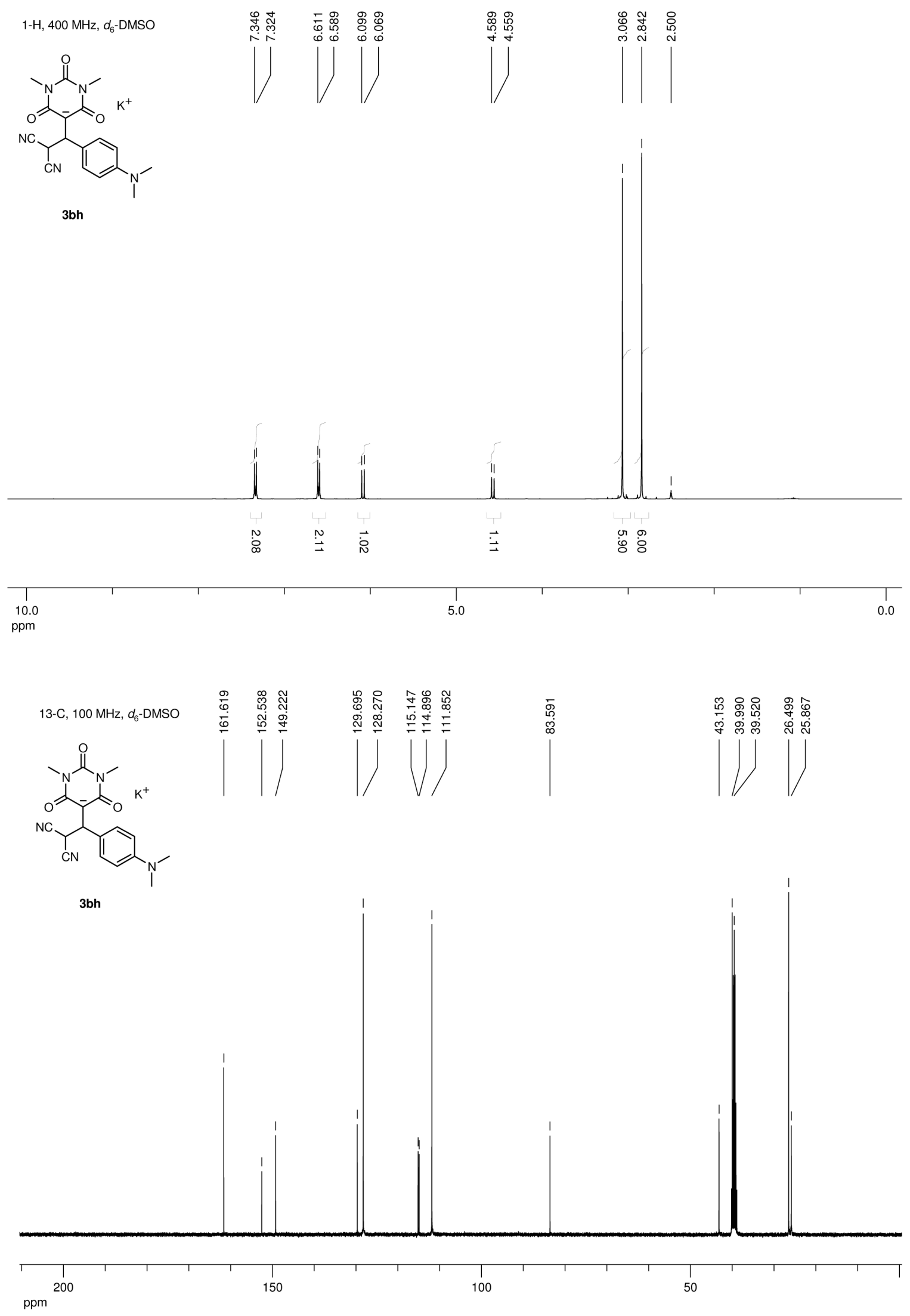


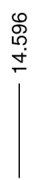

1-H, $400 \mathrm{MHz}, d_{6}-\mathrm{DMSO}$
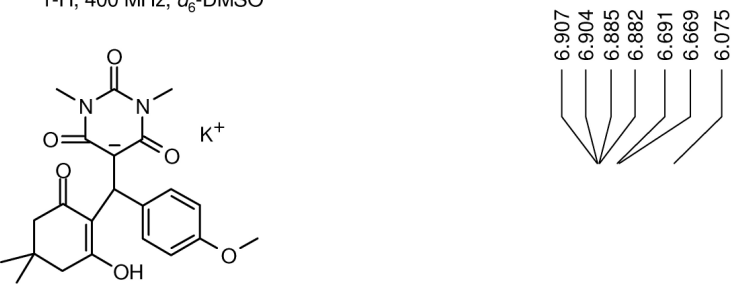

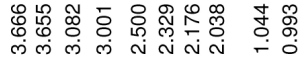

3cb

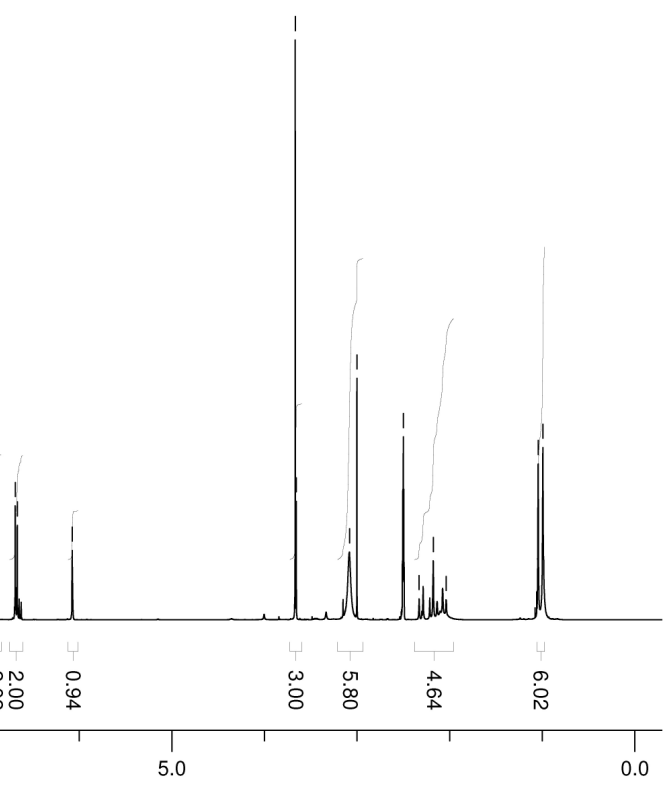

ppm

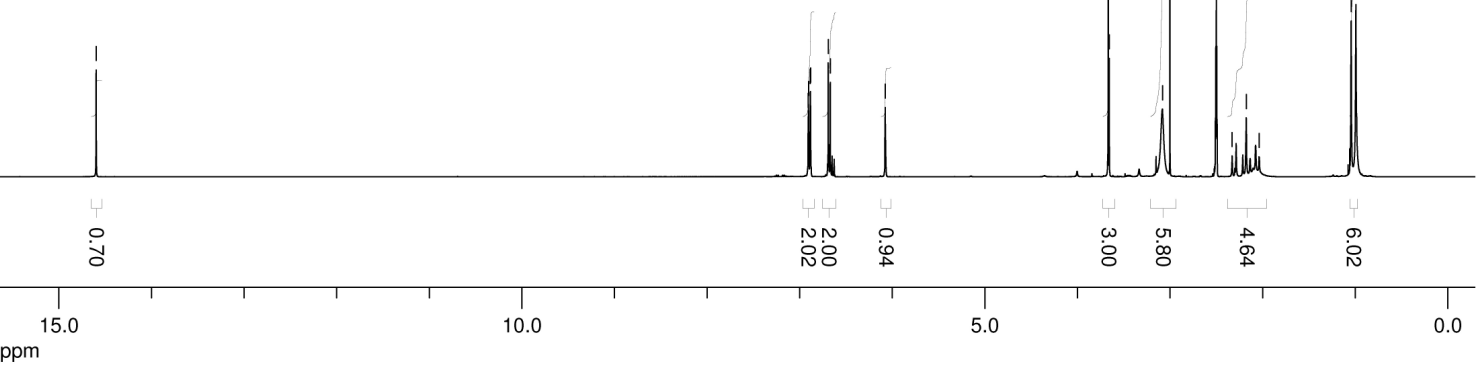

13-C, $75 \mathrm{MHz}, d_{6}$-DMSO
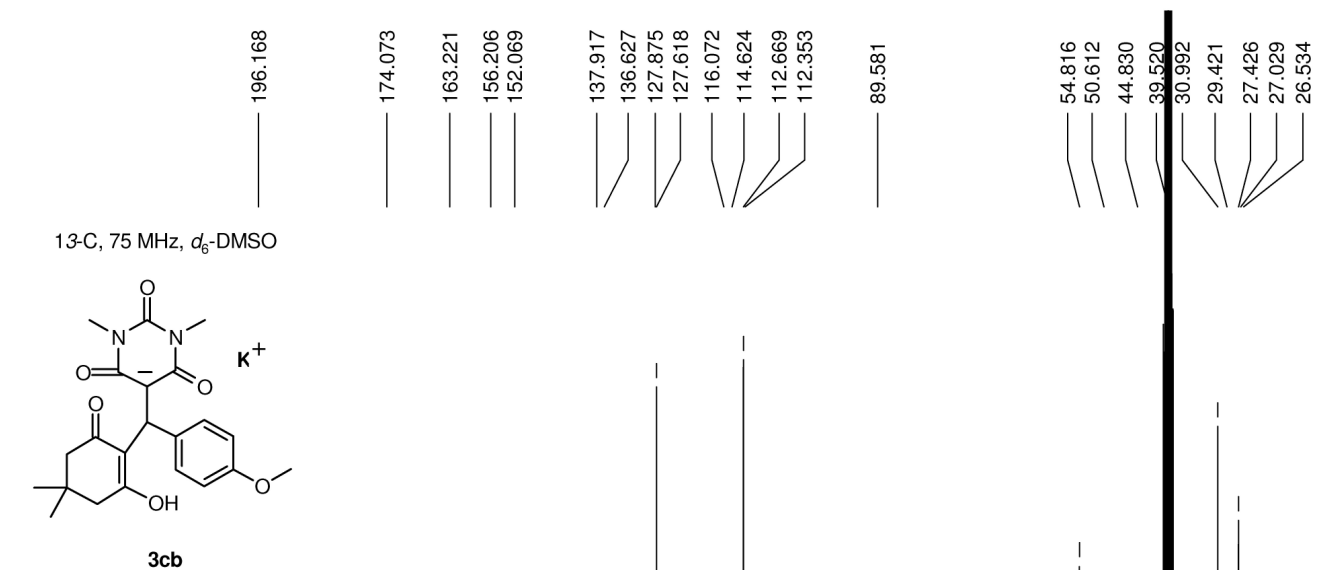

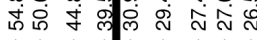

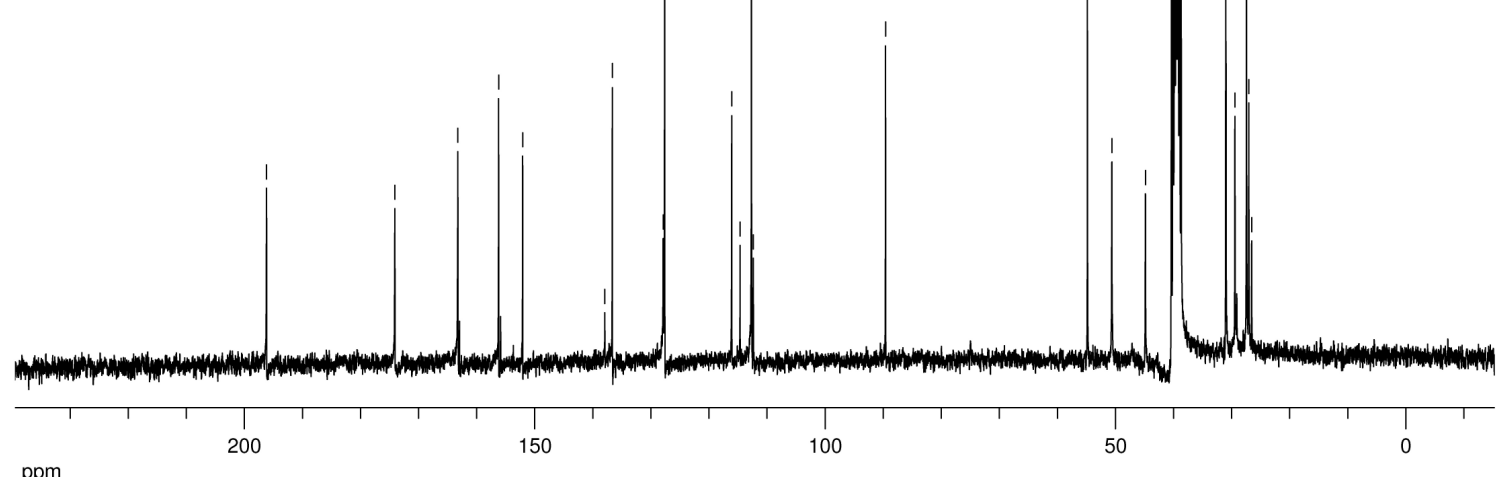


S17

1-H, $300 \mathrm{MHz}, d_{6}-\mathrm{DMSO}$
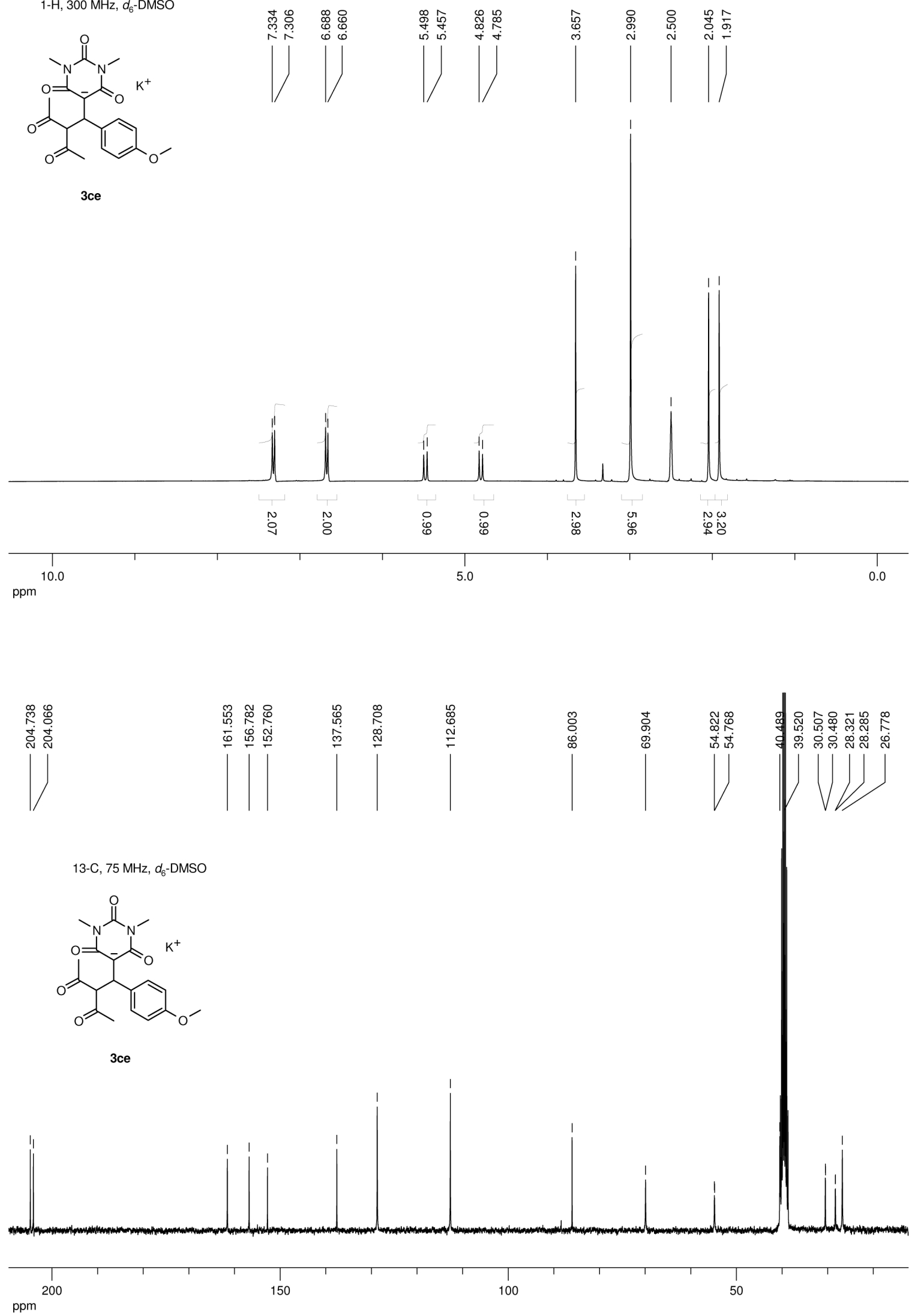

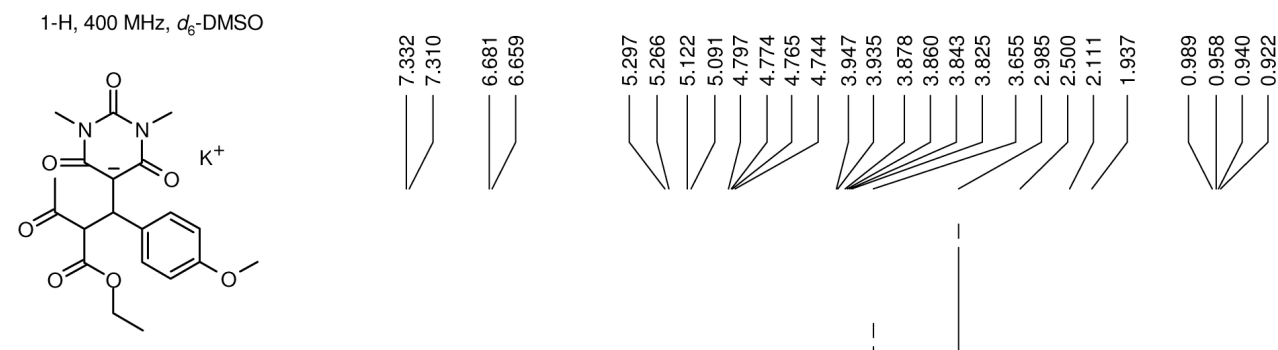

$3 \mathrm{cg}$
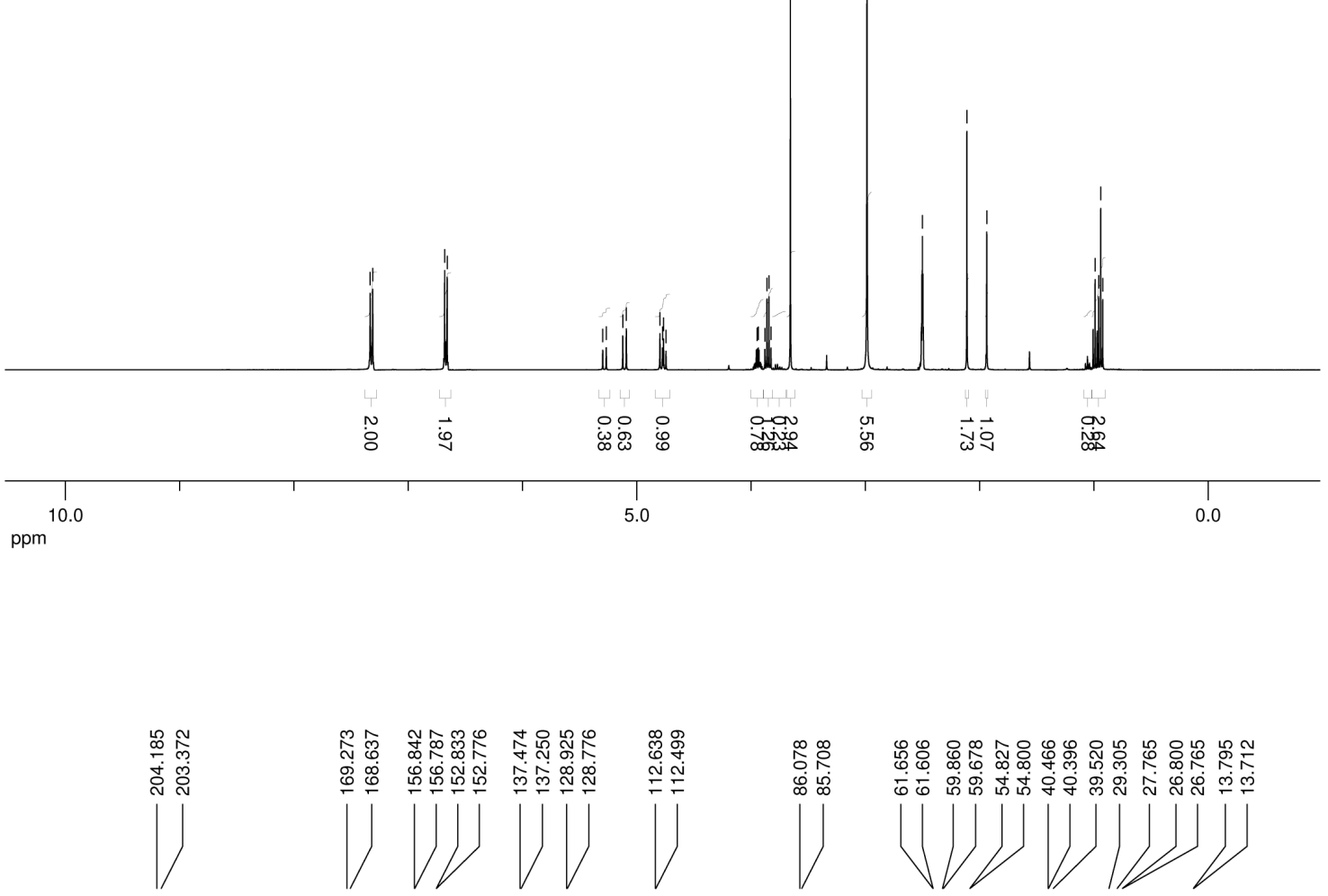

13-C, $100 \mathrm{MHz}, d_{6}$-DMSO

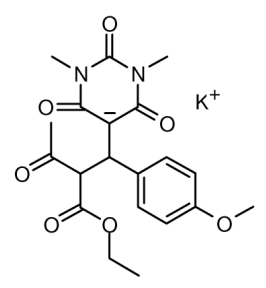

$3 \mathrm{cg}$

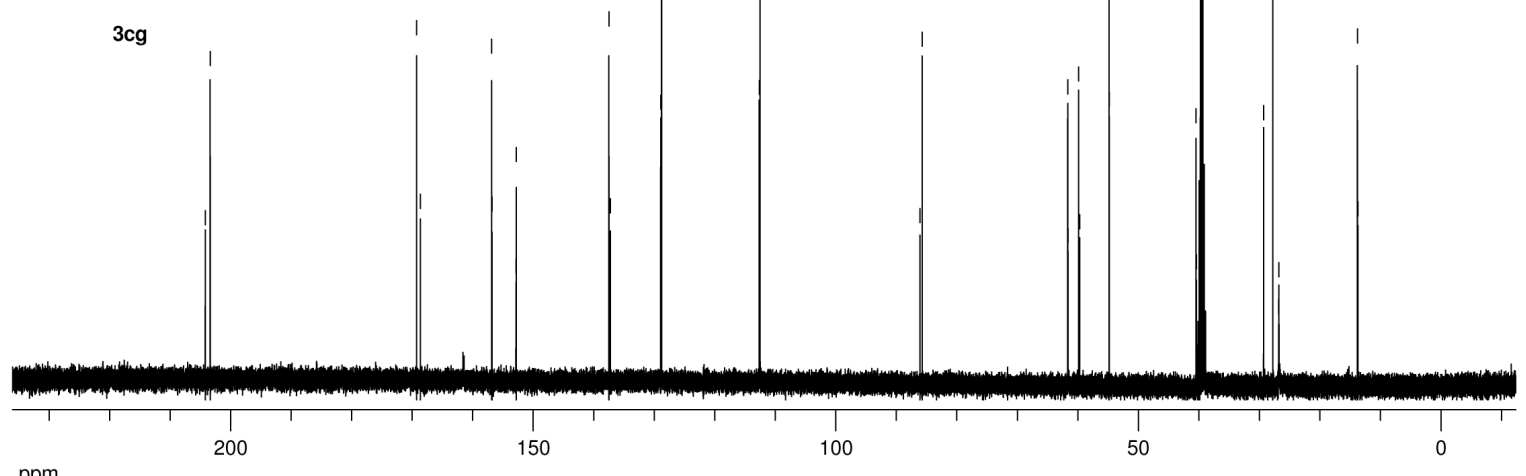


1-H, $400 \mathrm{MHz}, d_{6}$-DMSO

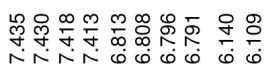

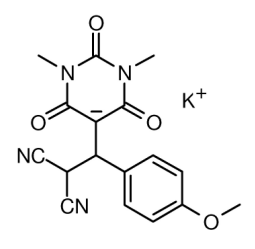
$\left(|l| l||^{0}\right.$

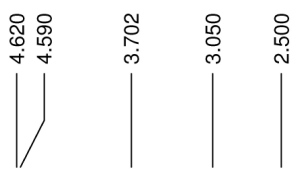

3ch

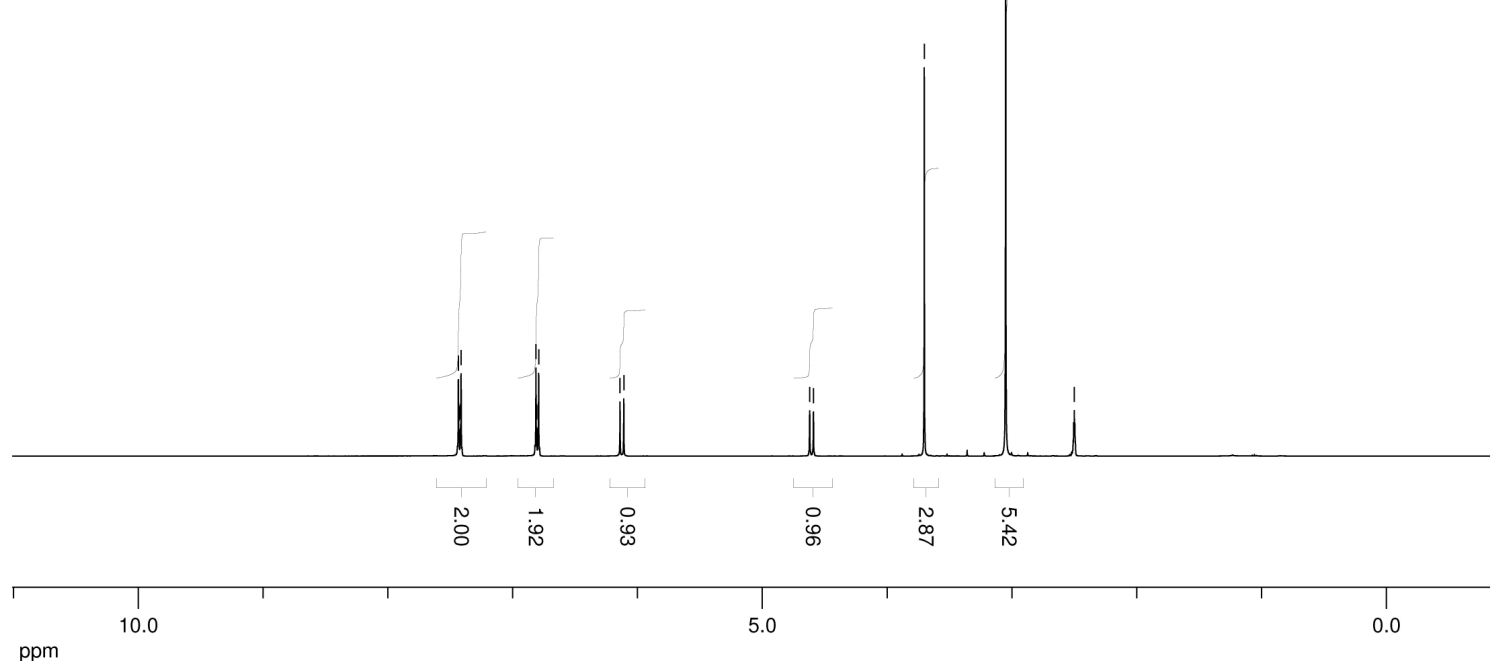

13-C, $100 \mathrm{MHz}, d_{6}$-DMSO
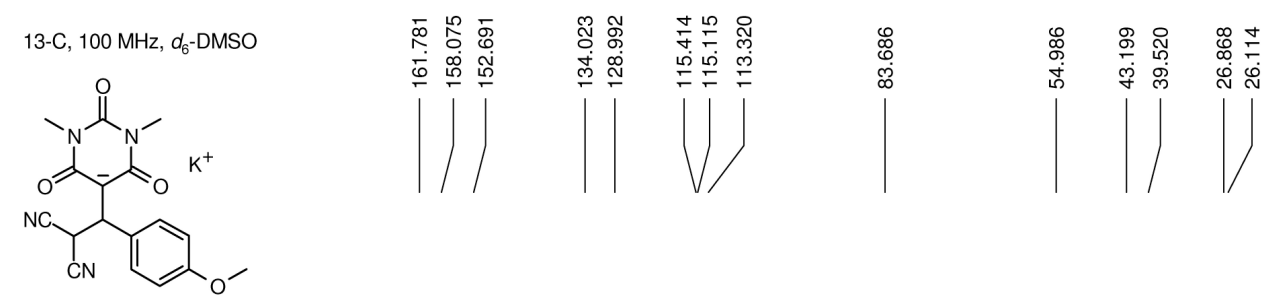

3ch

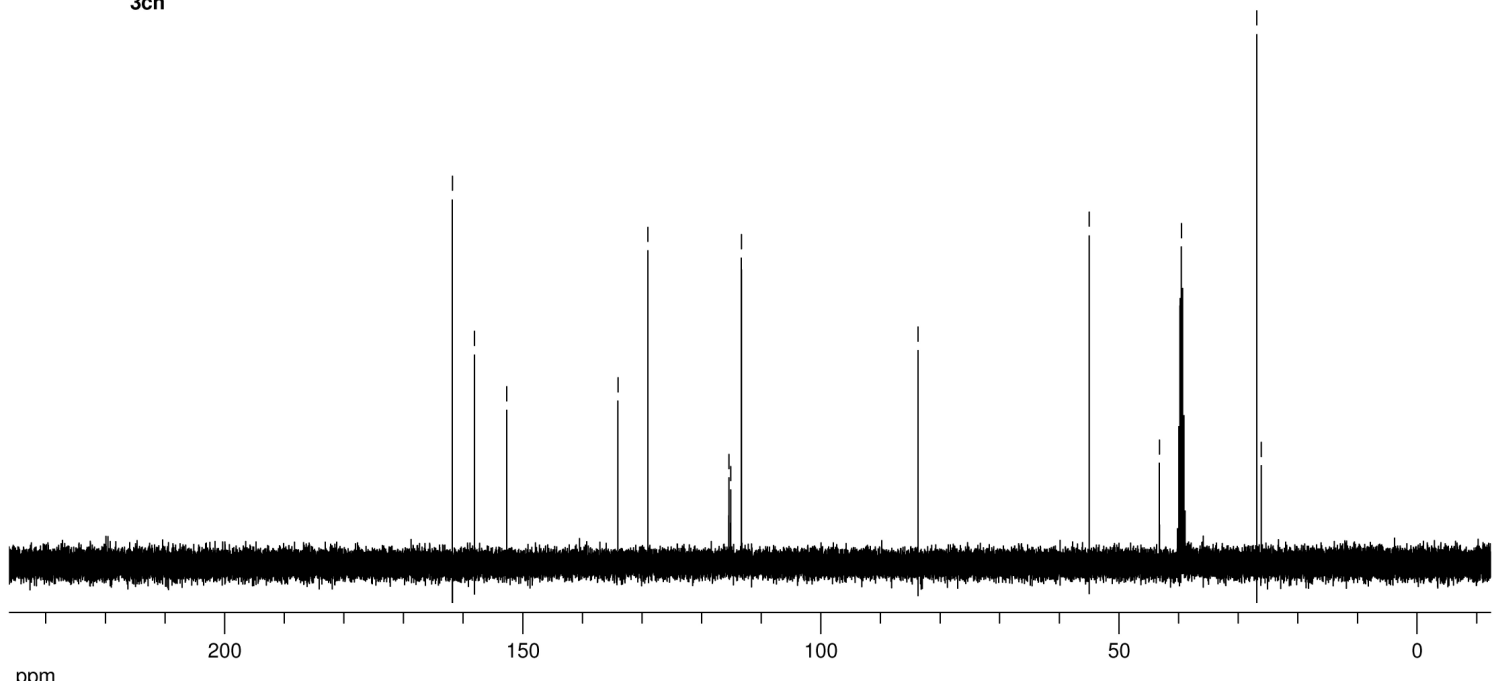



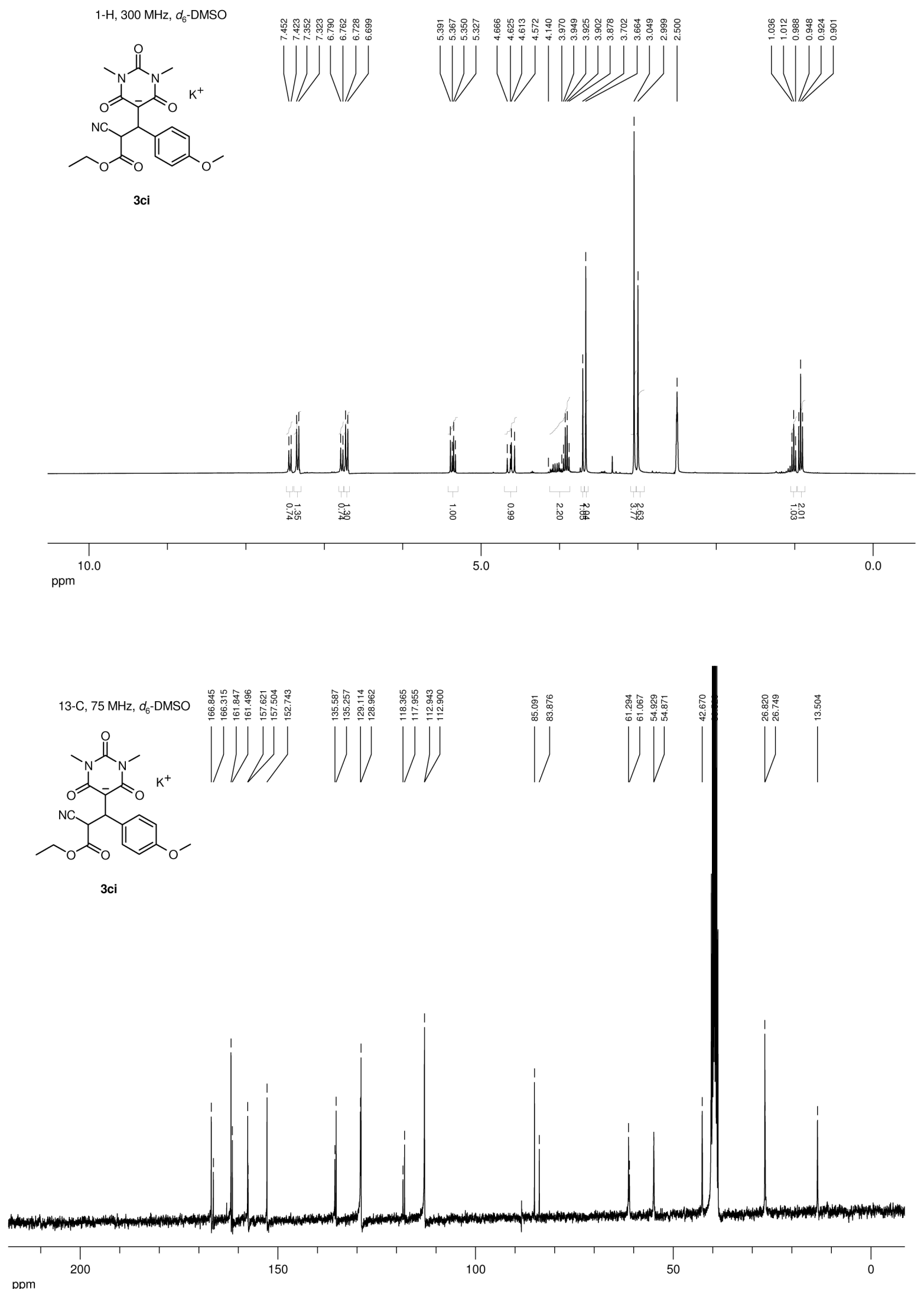


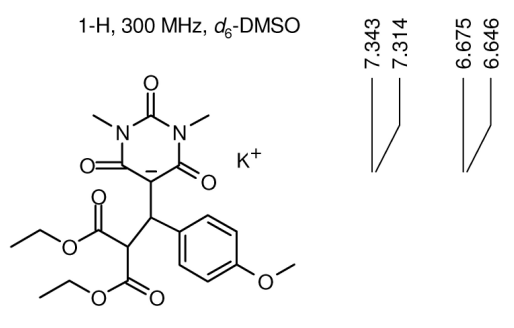

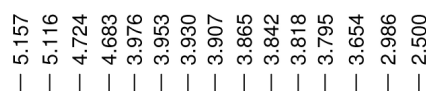

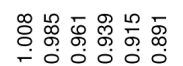

3ck
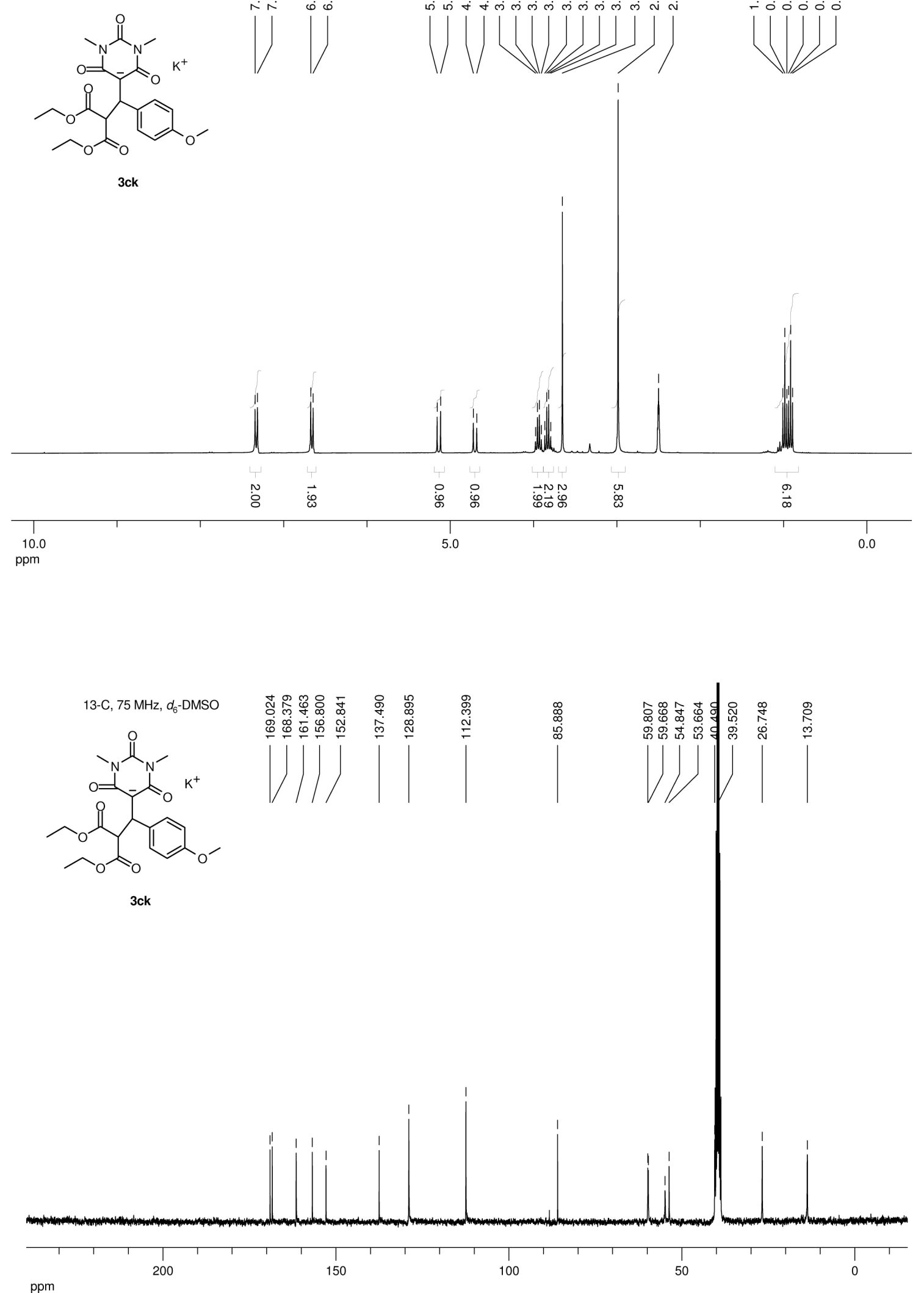
1-H, $300 \mathrm{MHz}, d_{6}$-DMSO<smiles></smiles>

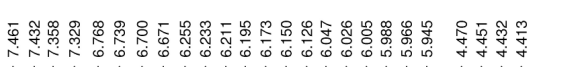

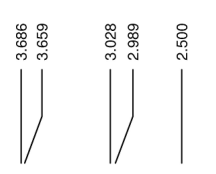

衰哭

$3 \mathrm{~cm}$

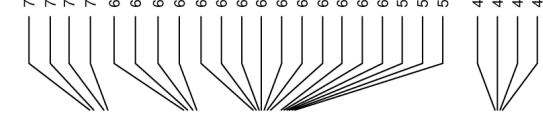

$11 \mid$
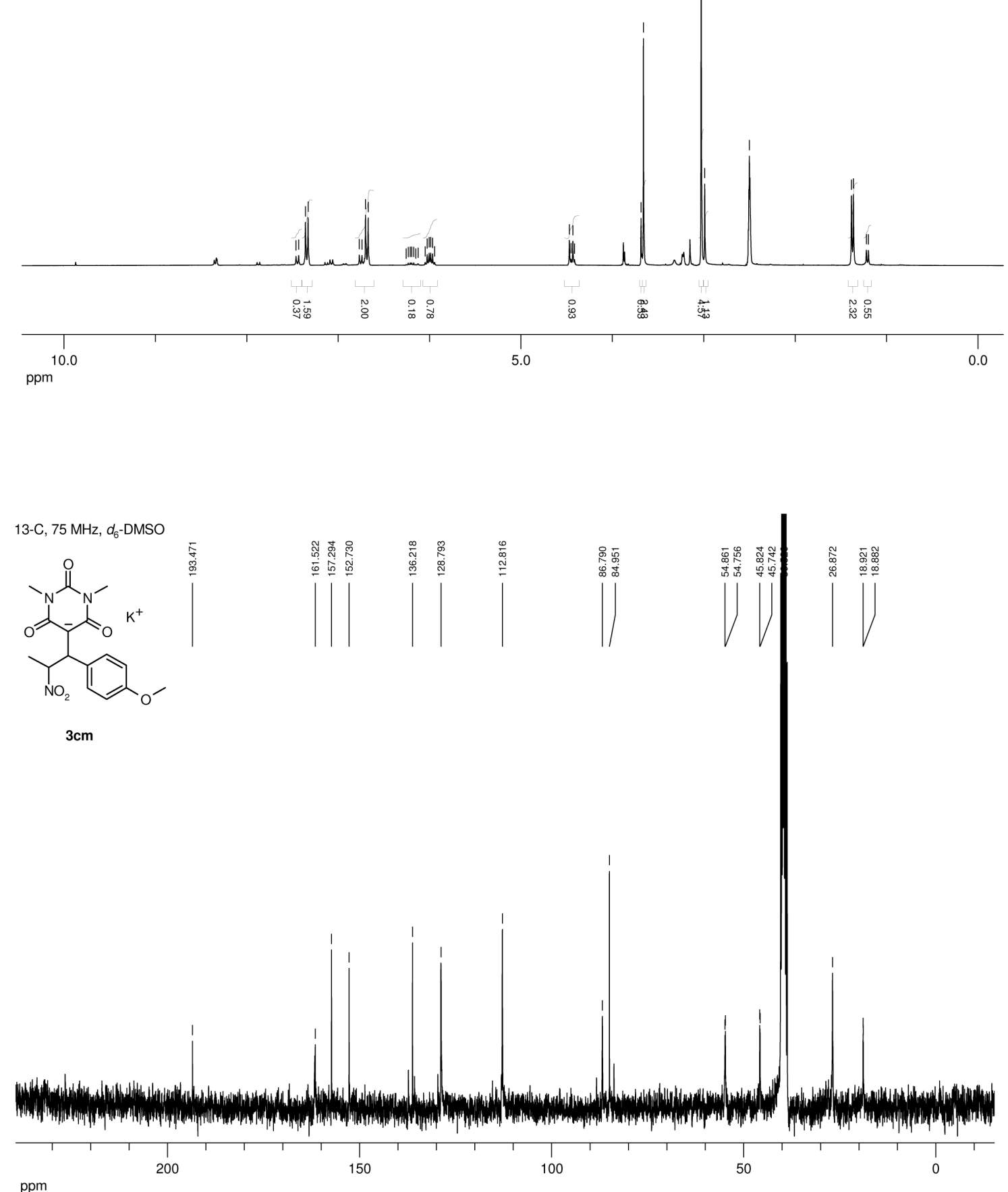

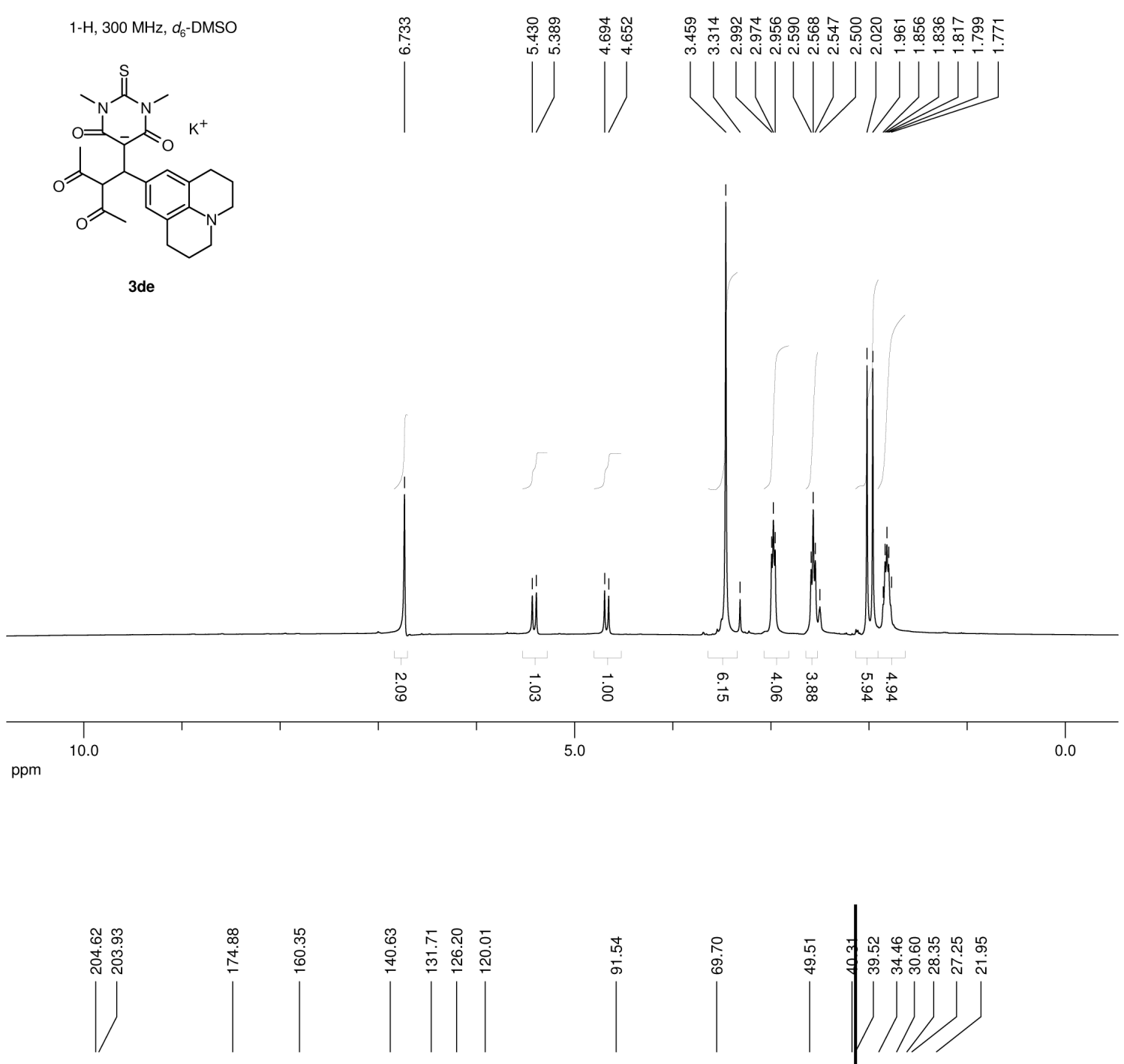

13-C, $100 \mathrm{MHz}, d_{6}$-DMSO<smiles></smiles>

3de

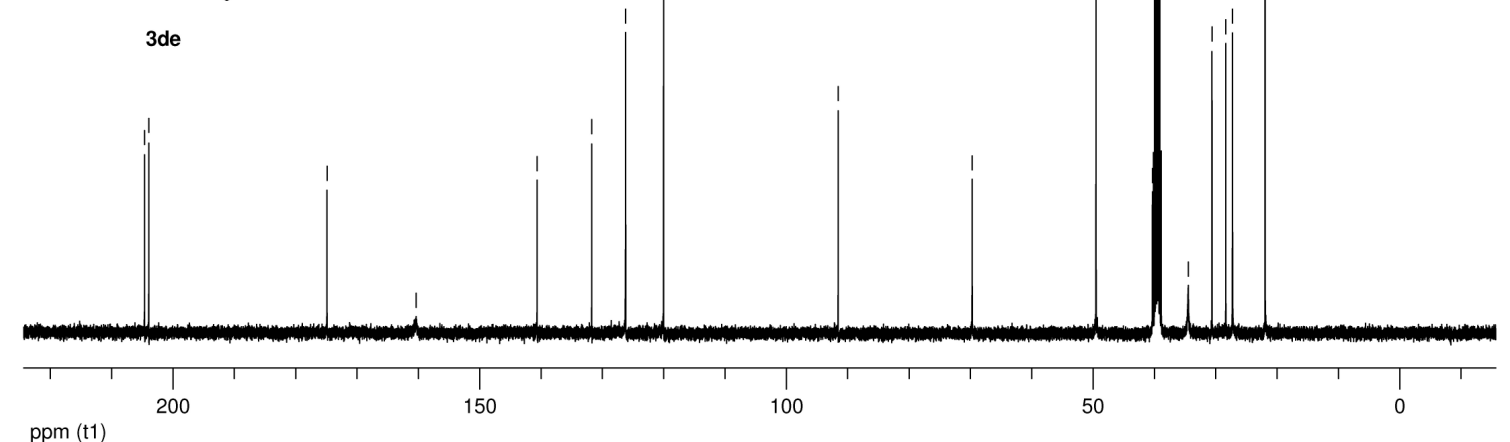



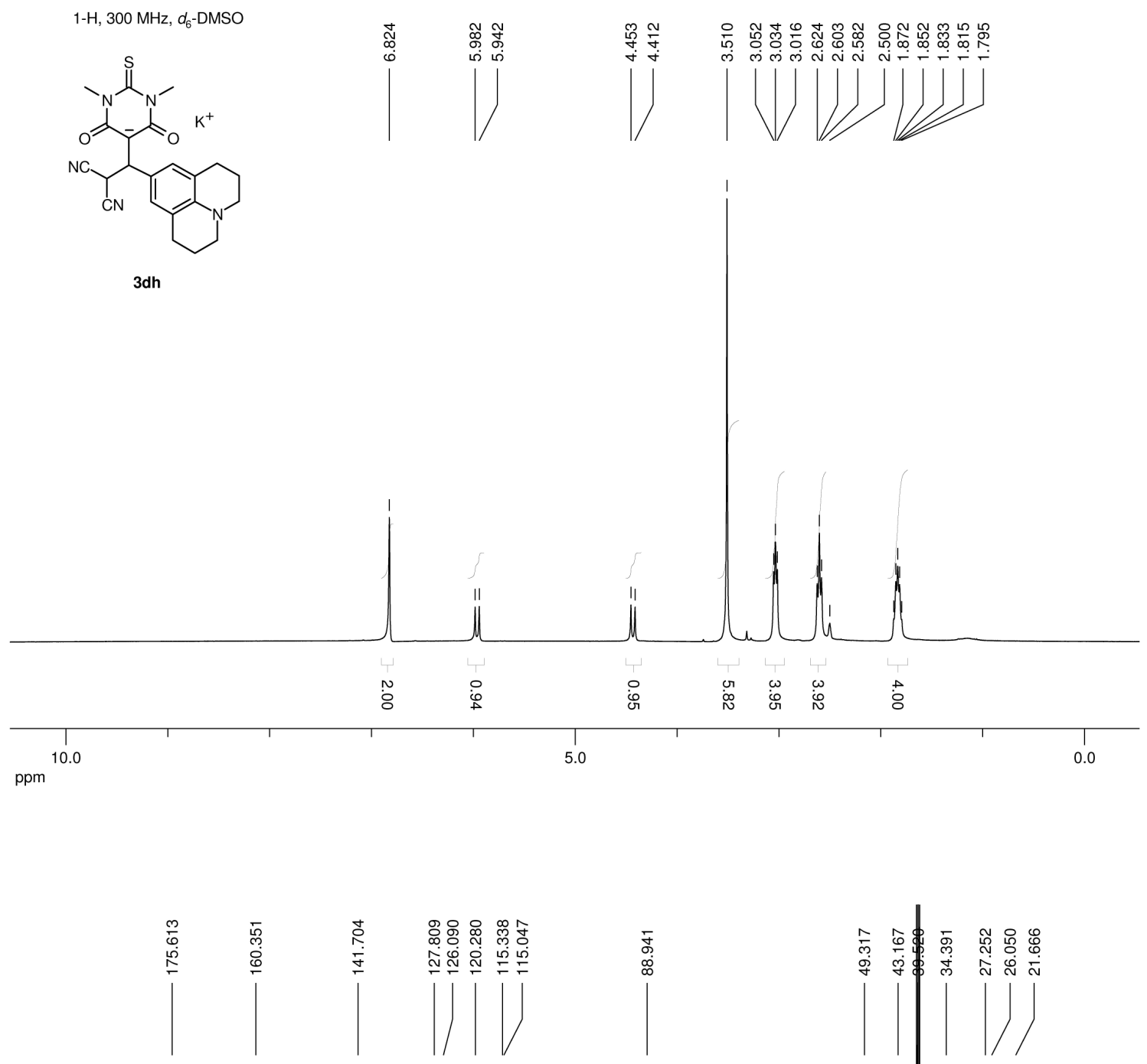

13-C, $75 \mathrm{MHz}, d_{6}$-DMSO

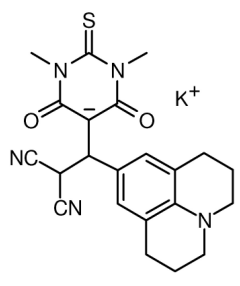

3dh

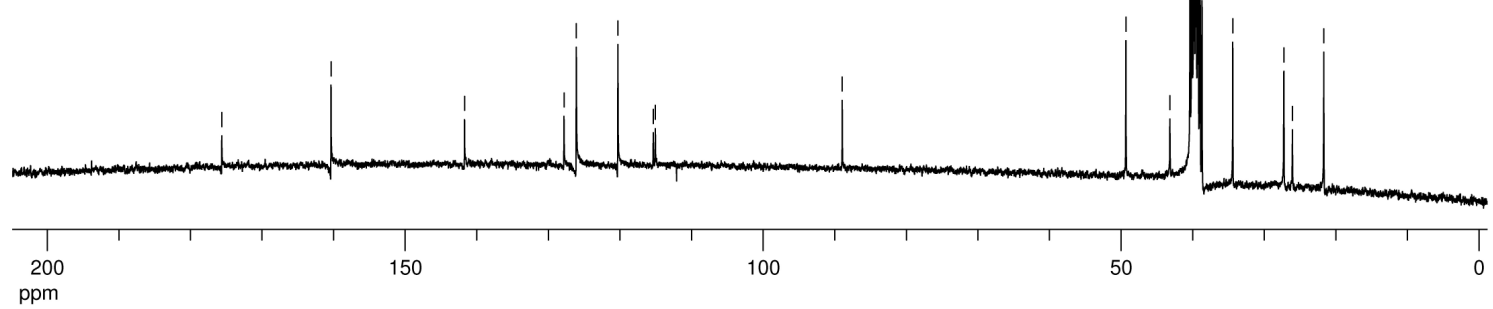


S25
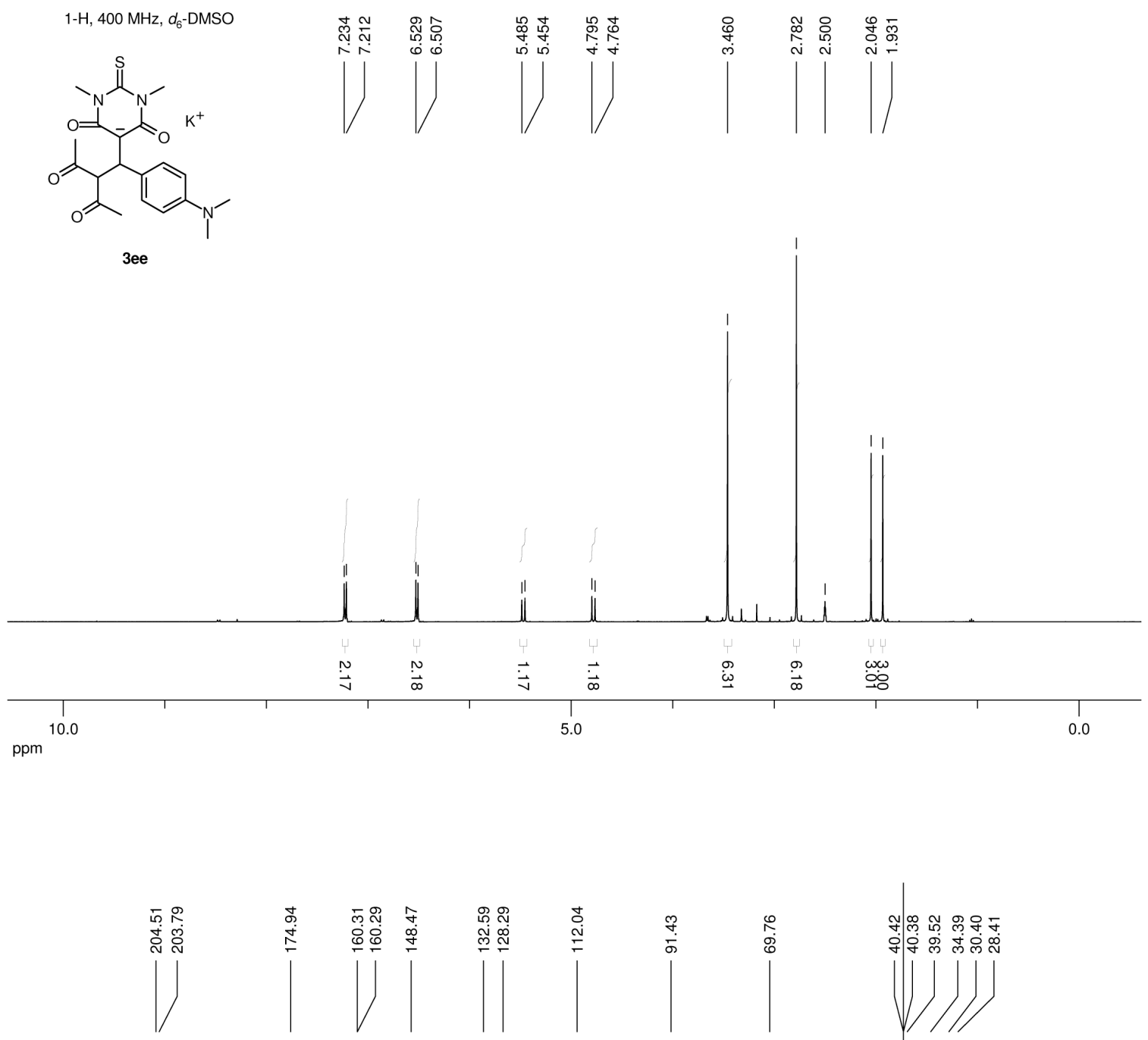

13-C, $100 \mathrm{MHz}, d_{6}$-DMSO
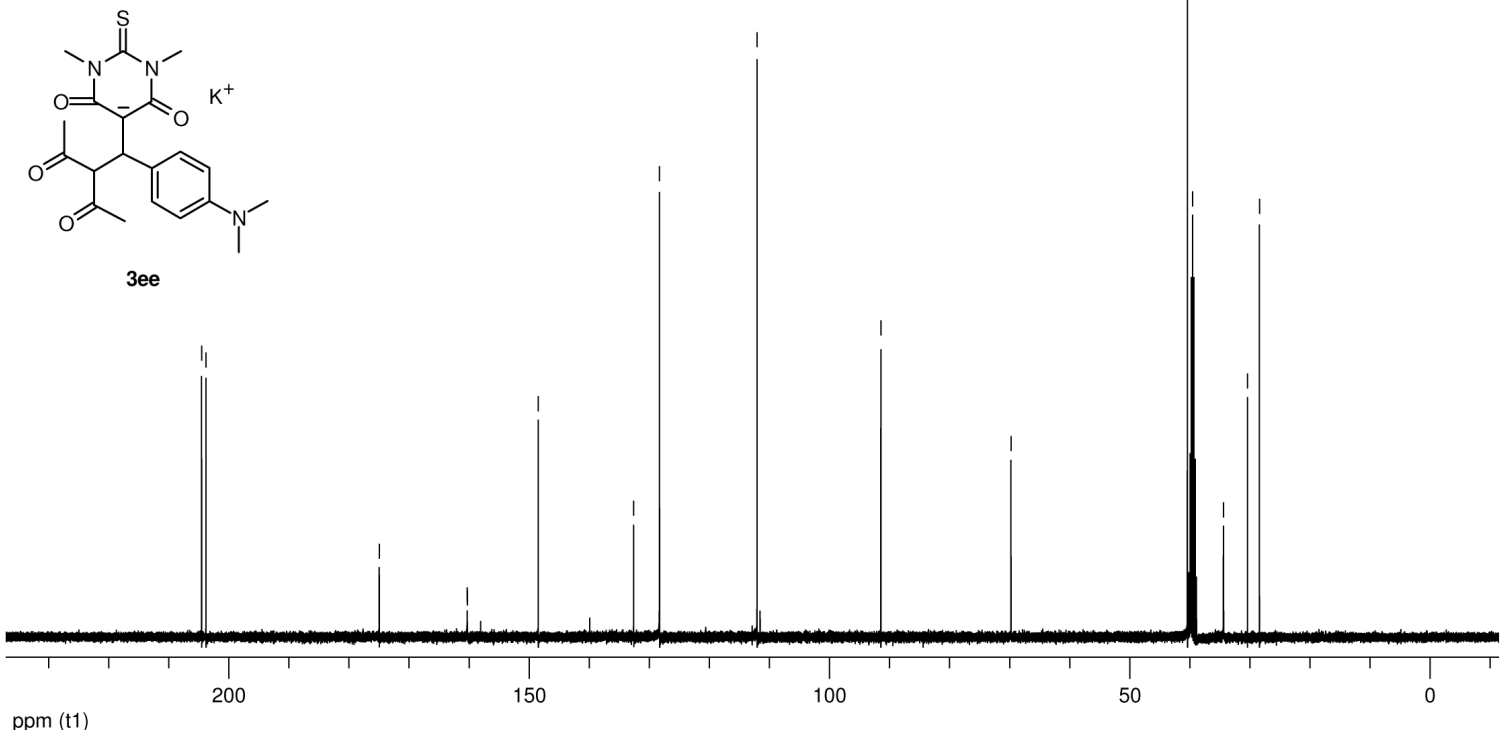
nmr of products 4
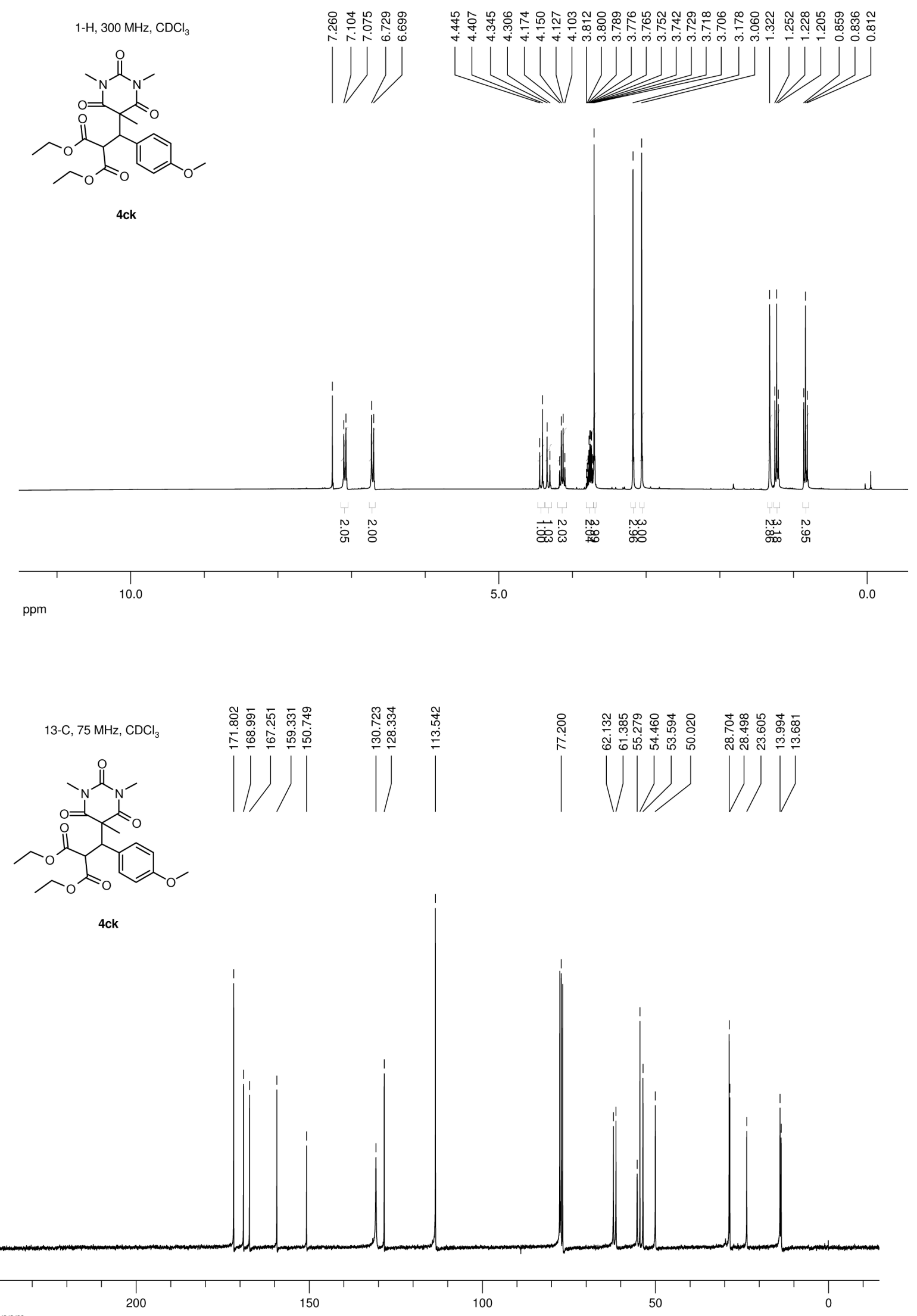
S27
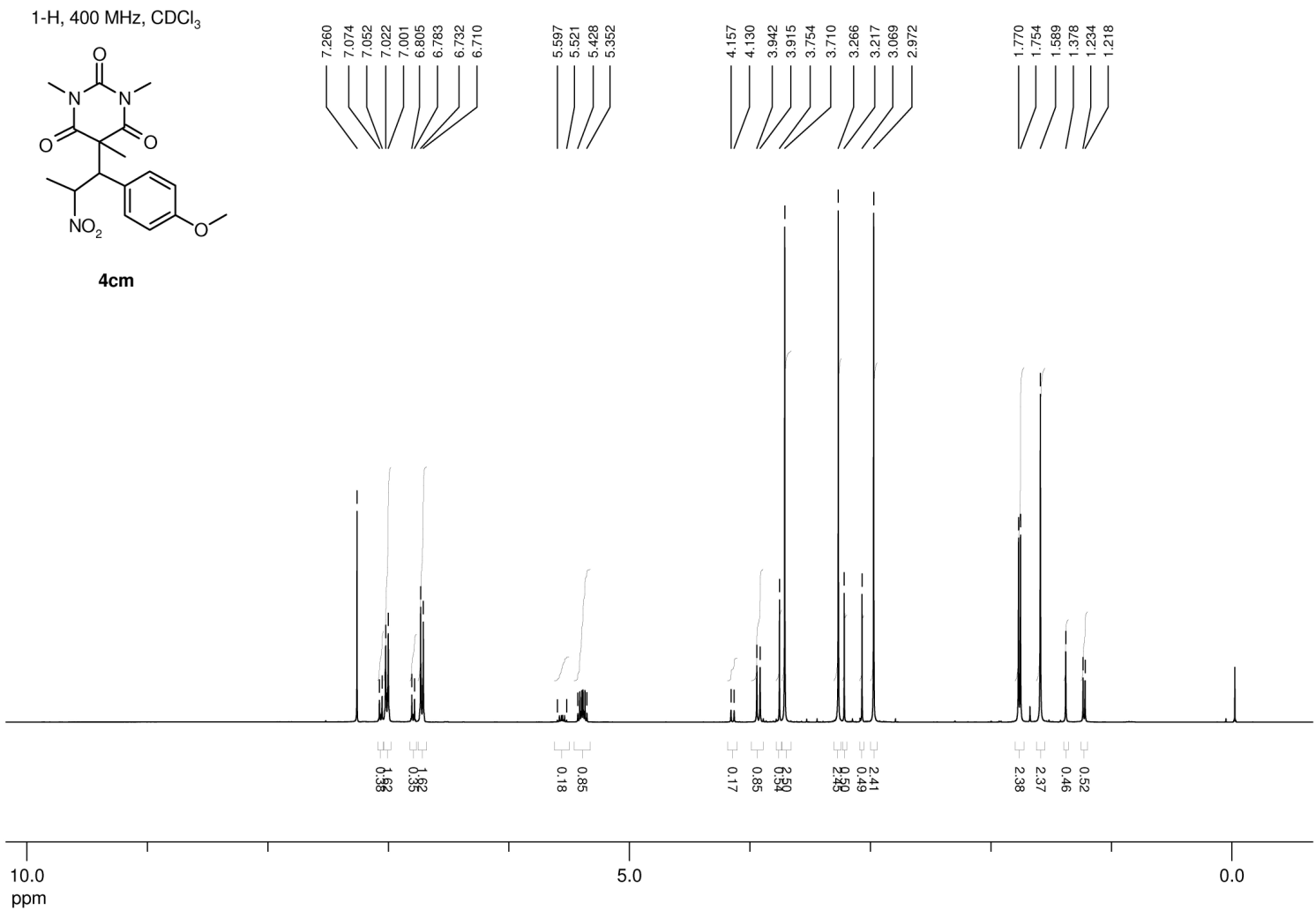

13-C, $75 \mathrm{MHz}, \mathrm{CDCl}_{3}$

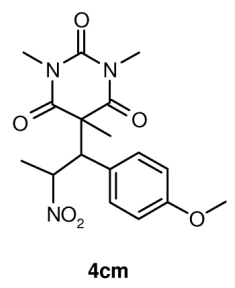

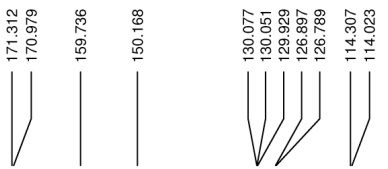
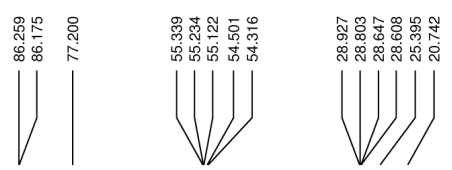

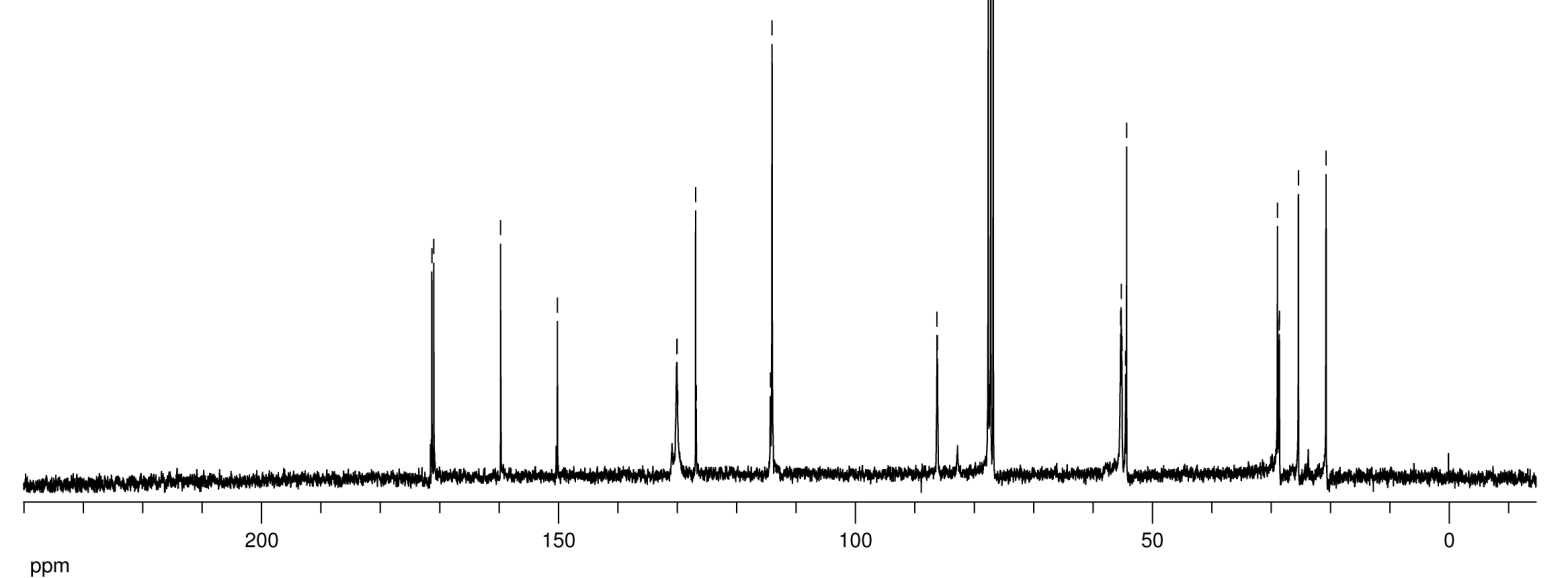

ppm 
S28

nmr of products 5
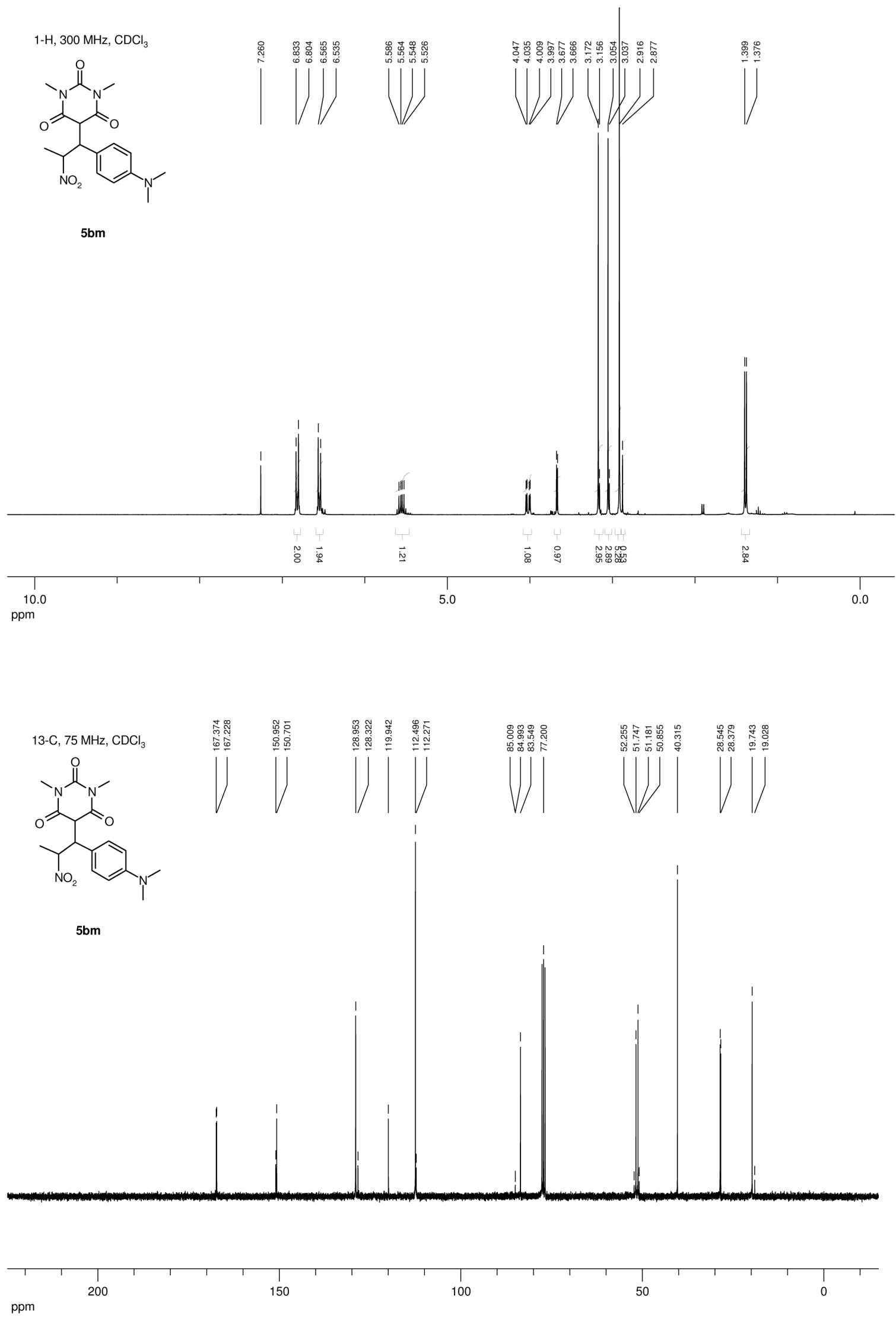

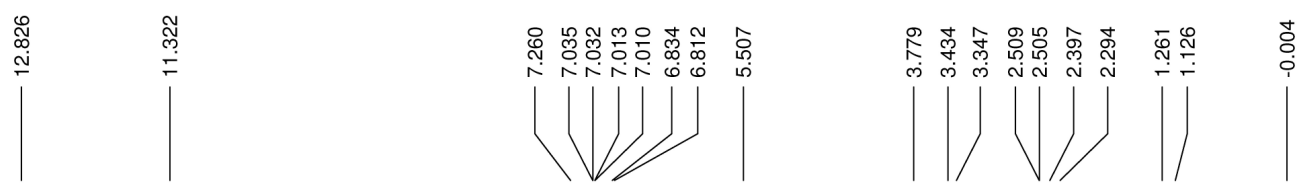

1-H, $400 \mathrm{MHz}, \mathrm{CDCl}_{3}$
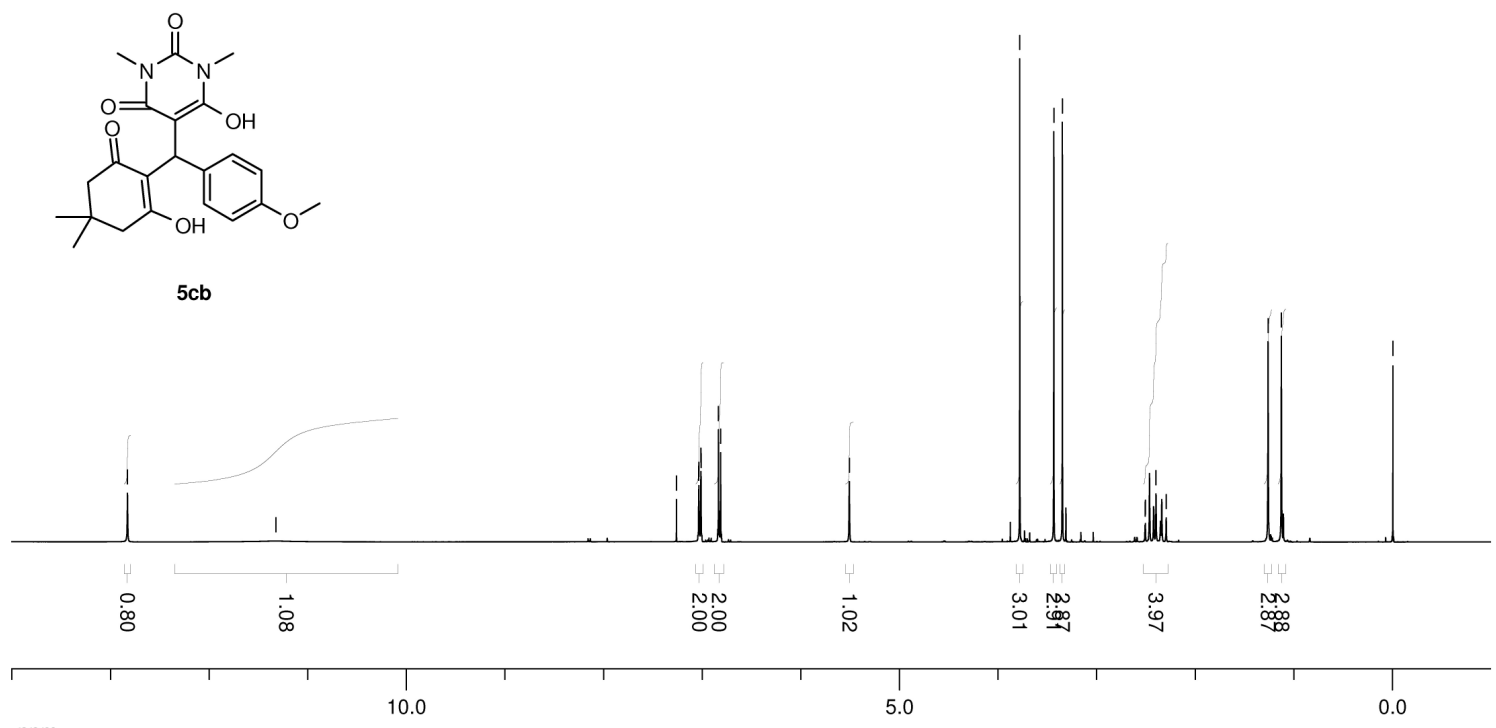

ppm

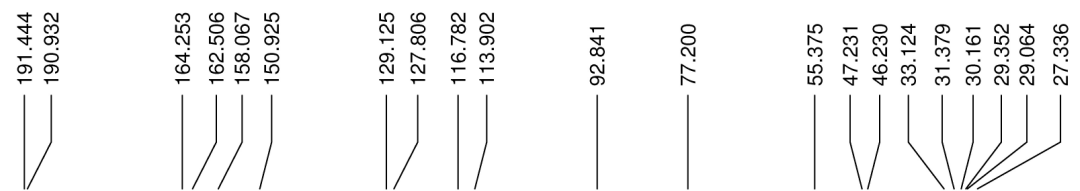

13-C, $75 \mathrm{MHz}, \mathrm{CDCl}_{3}$
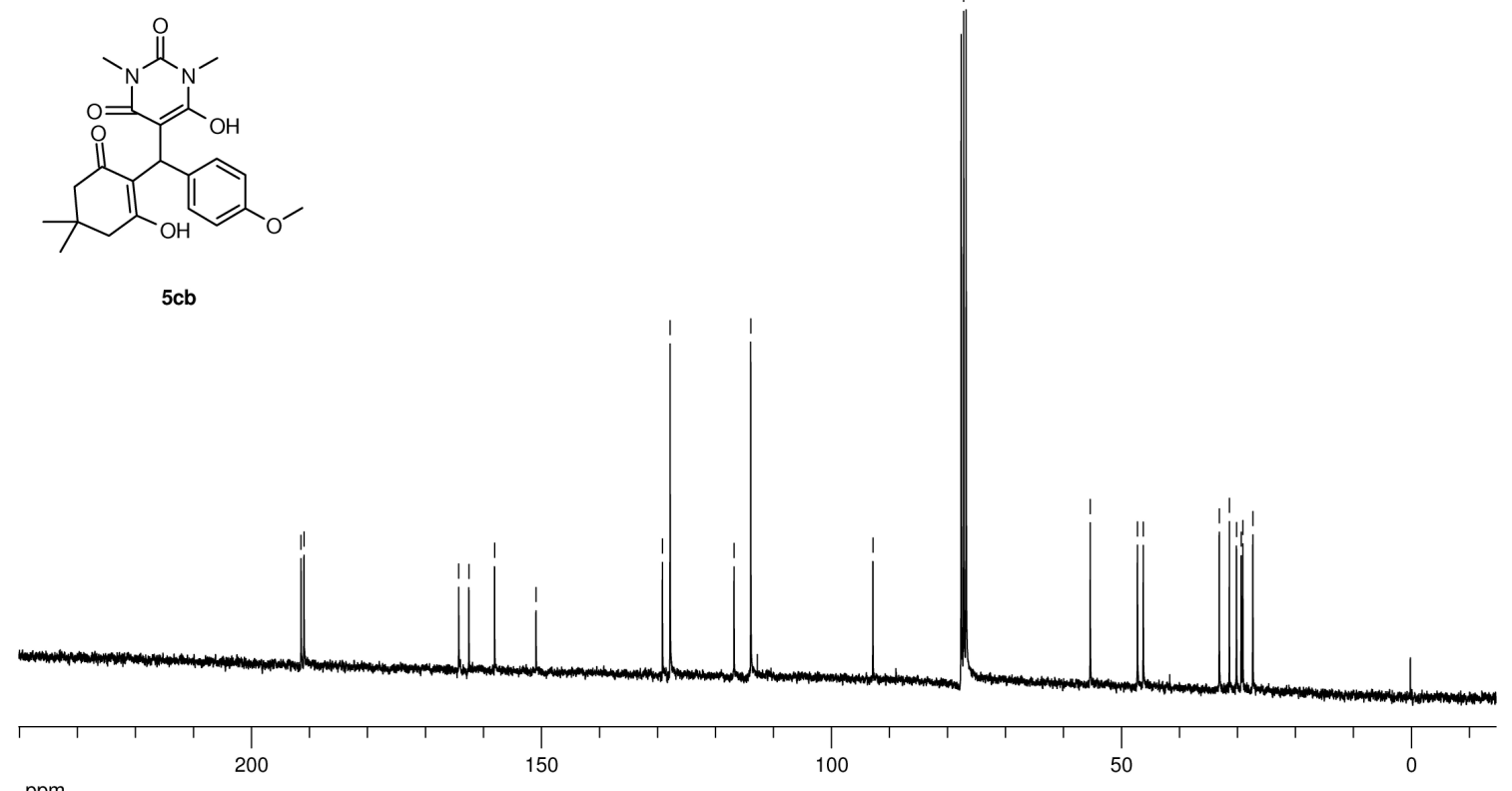

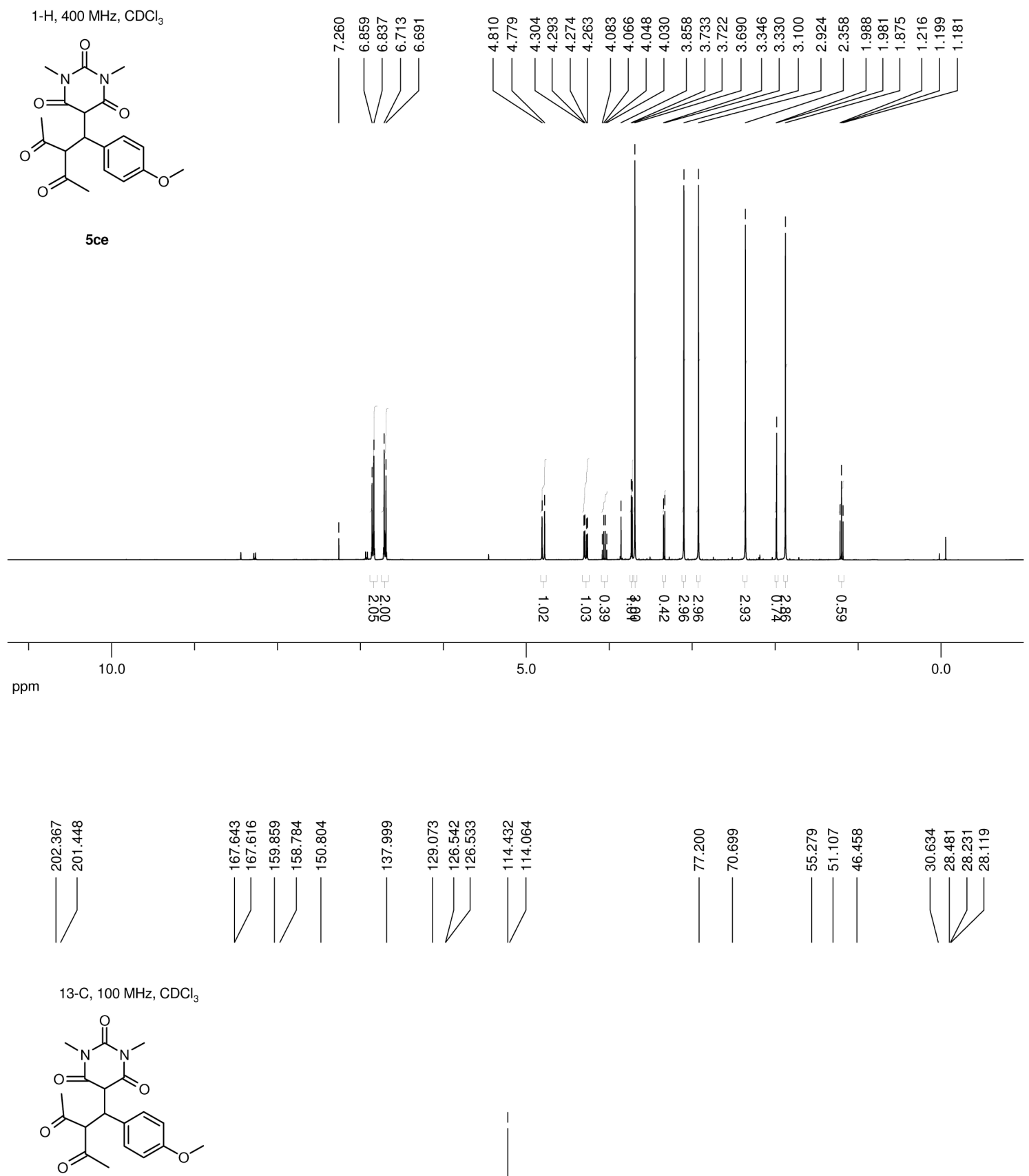

$5 c e$

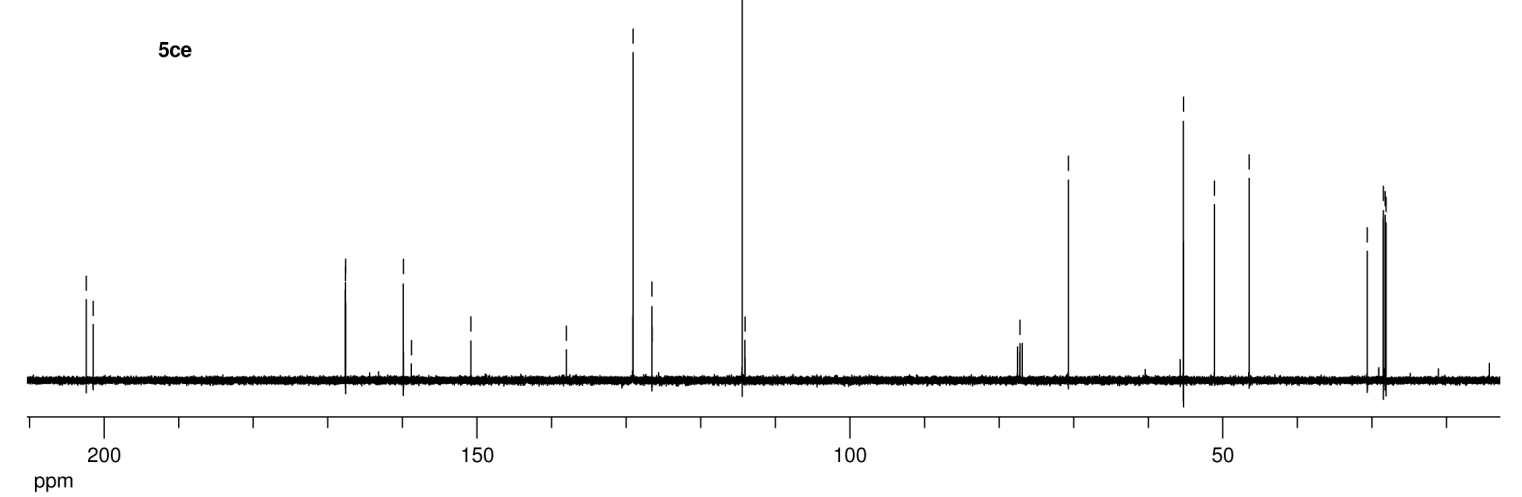



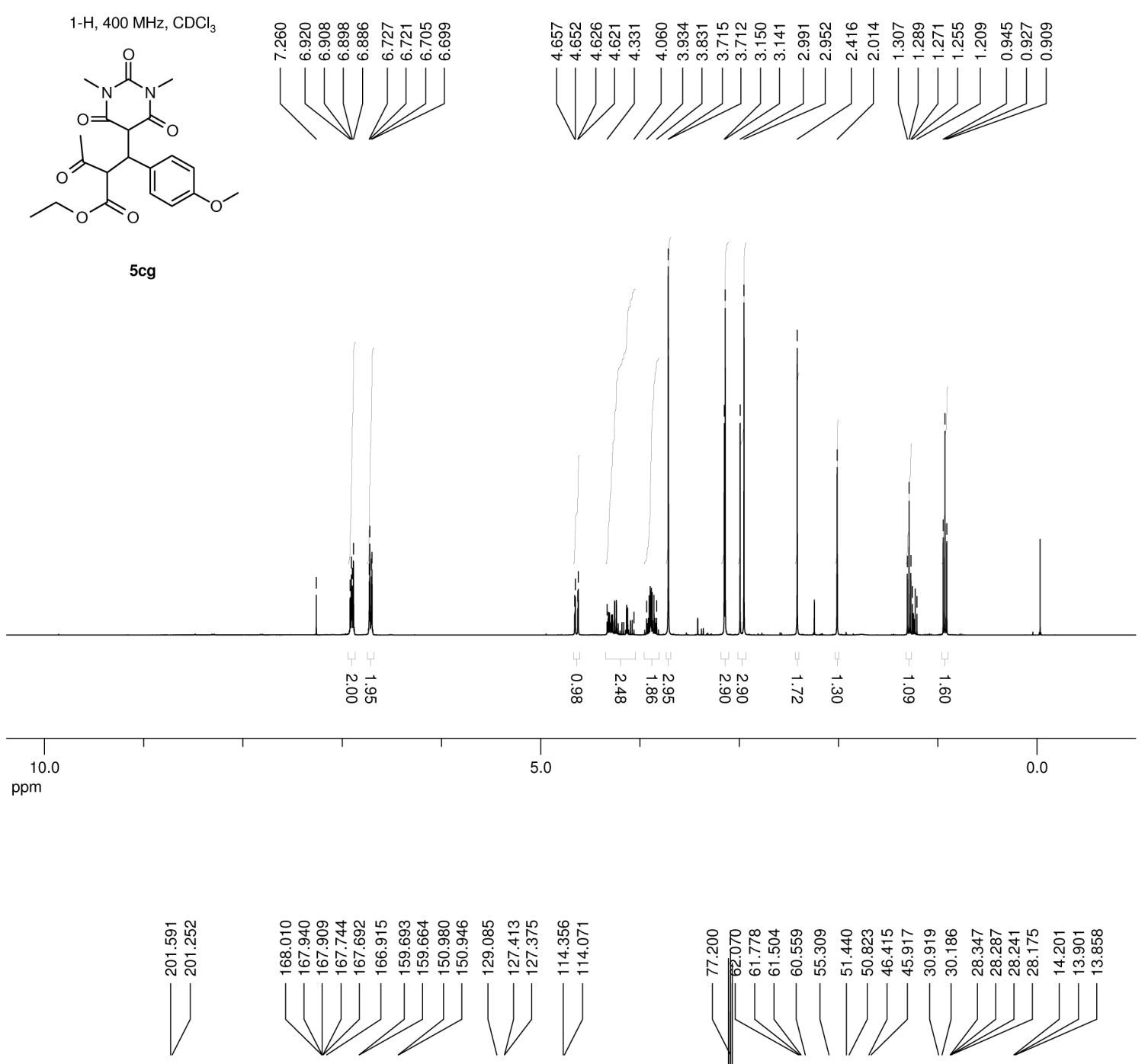

13-C, $75 \mathrm{MHz}, \mathrm{CDCl}_{3}$

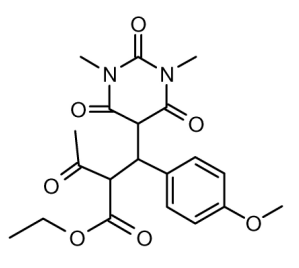

$5 \mathrm{cg}$

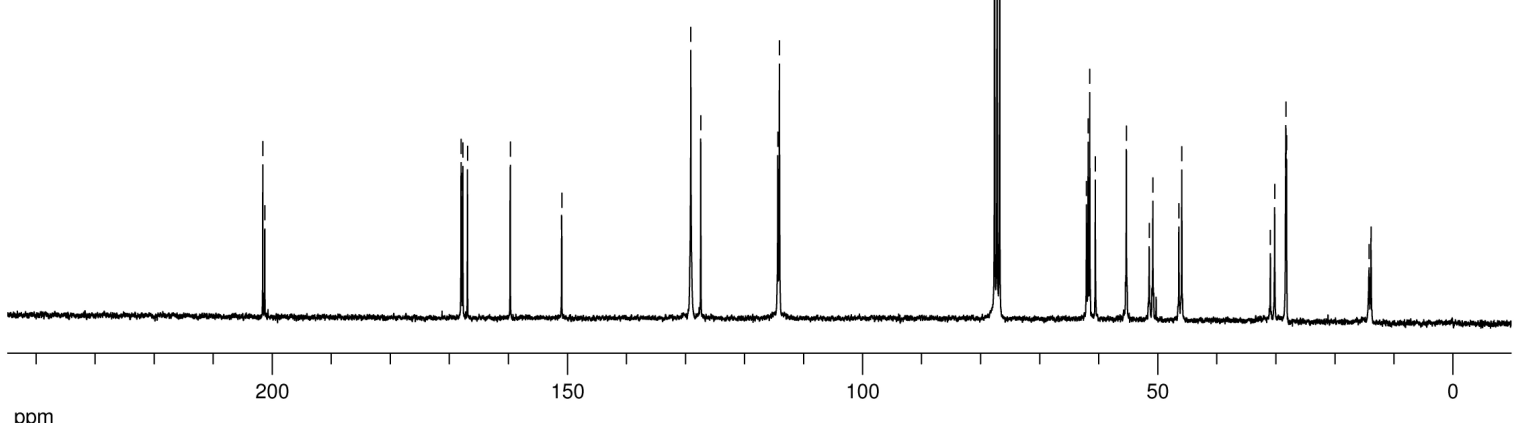


S32
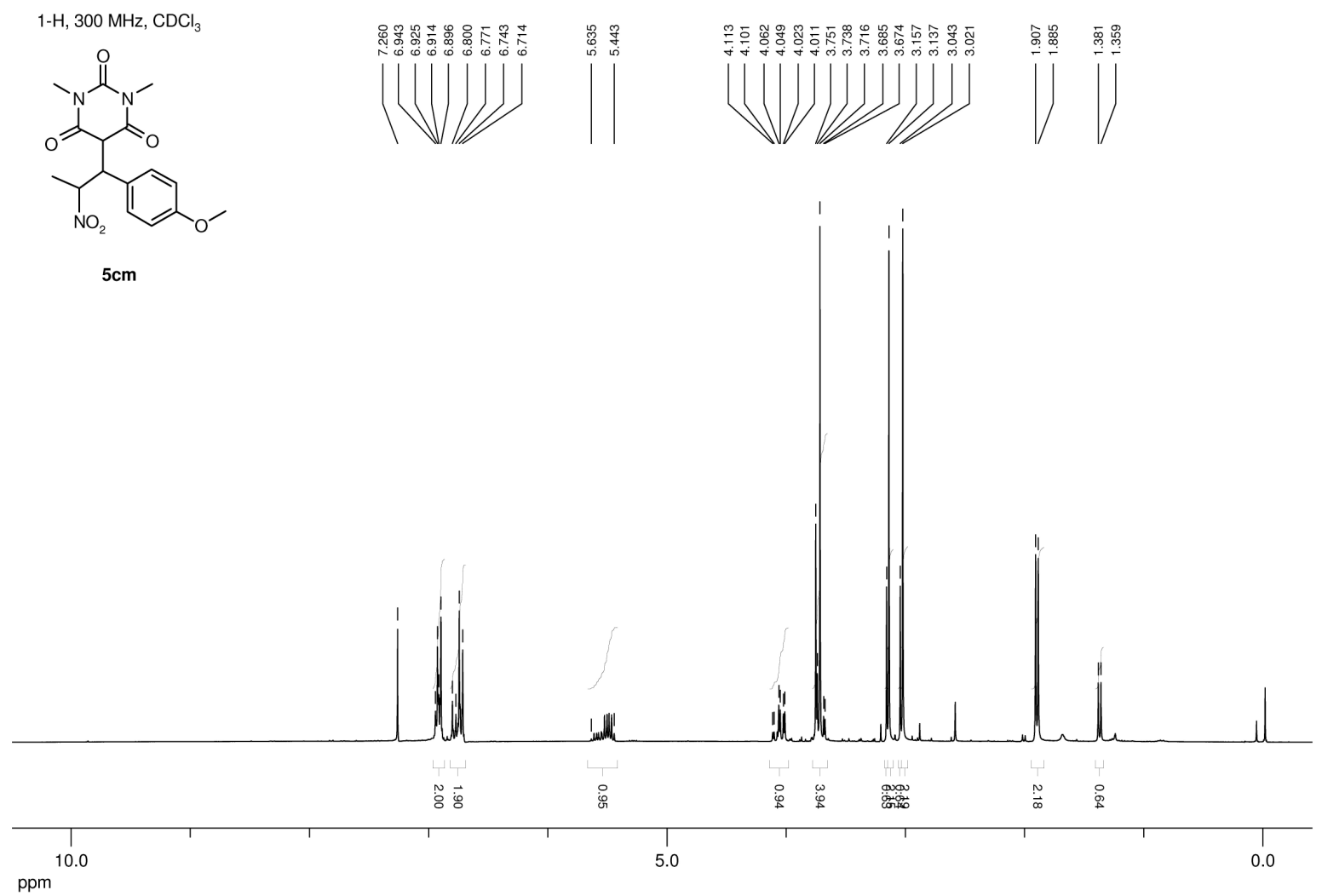

13-C, $75 \mathrm{MHz}, \mathrm{CDCl}_{3}$
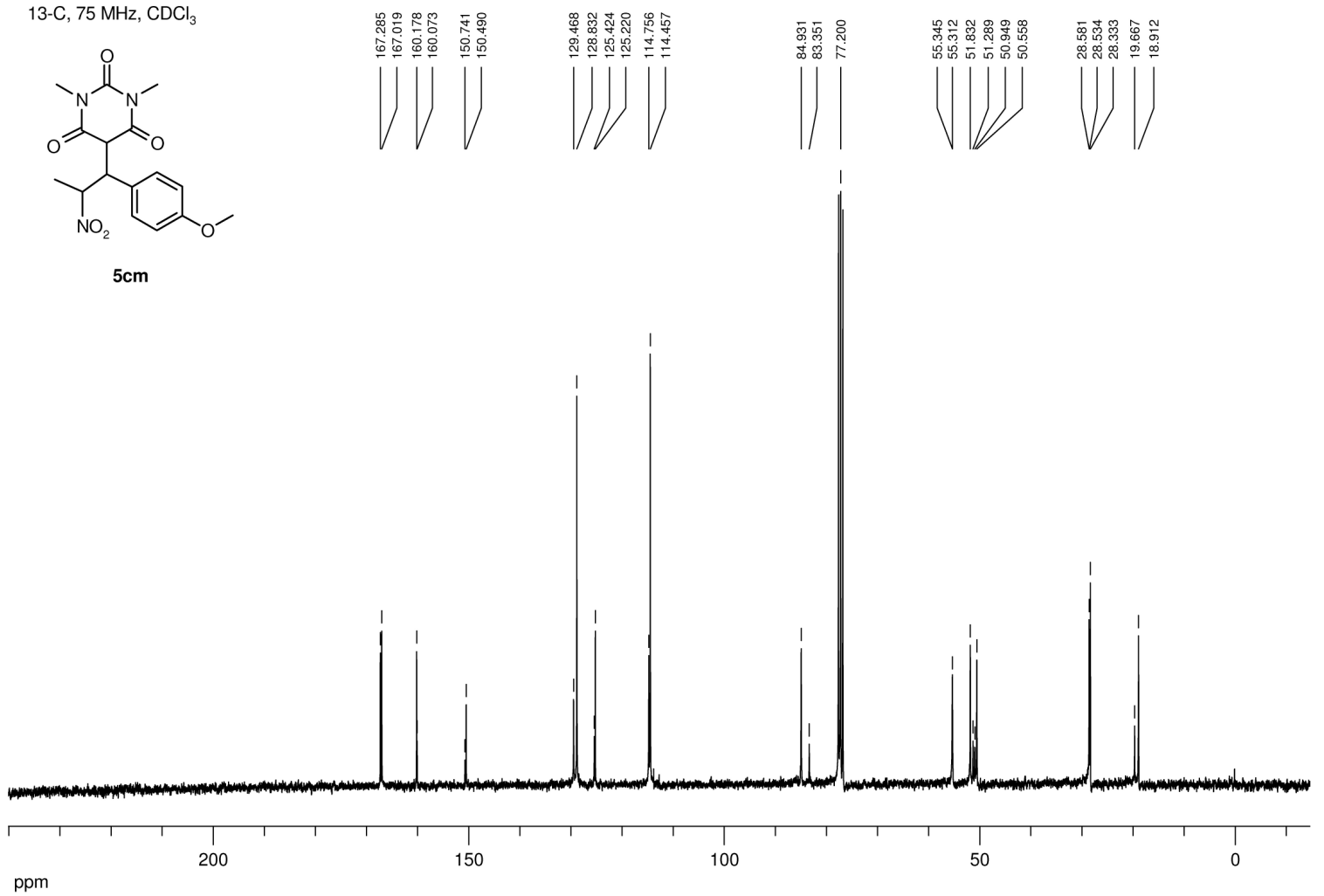


\section{nmr of $8 \mathrm{ch}$}
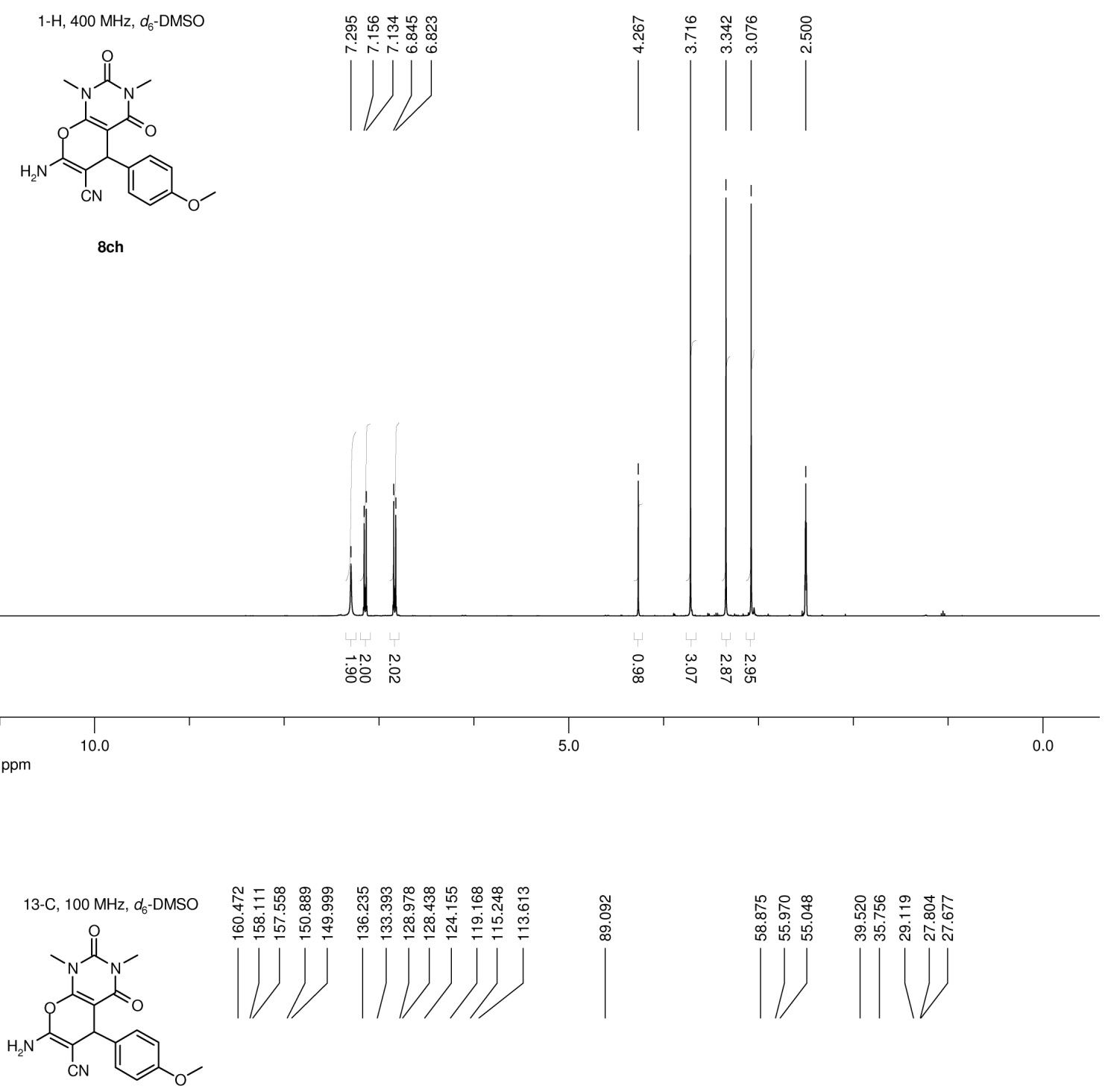

8ch

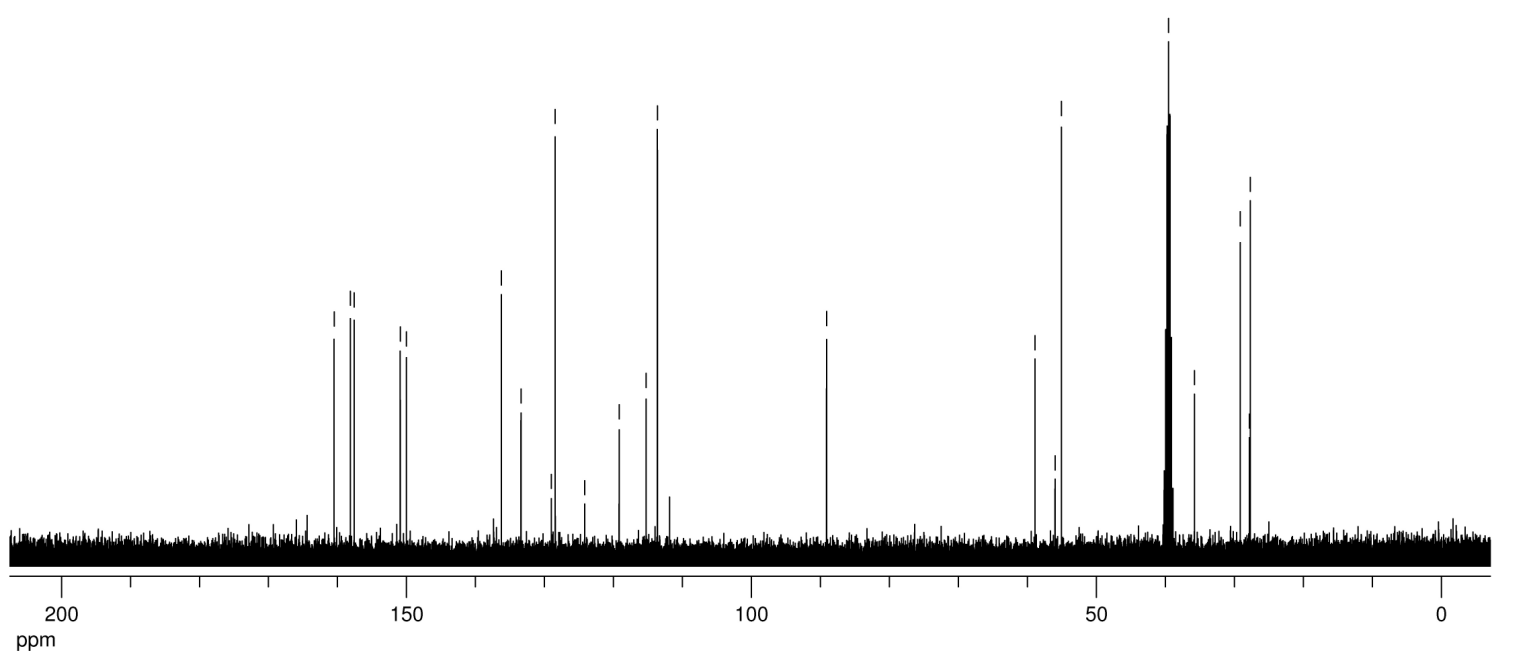


S34

nmr of 9ch

1- $\mathrm{H}, 600 \mathrm{MHz}, \mathrm{CDCl}_{3}$
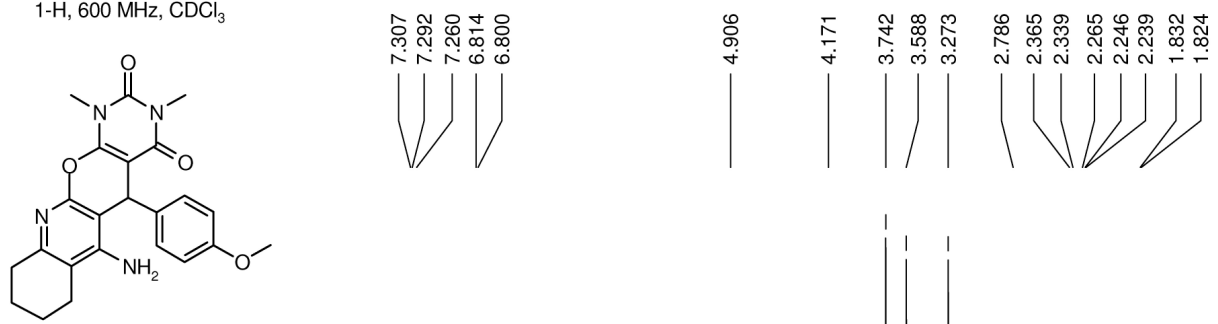

9ch
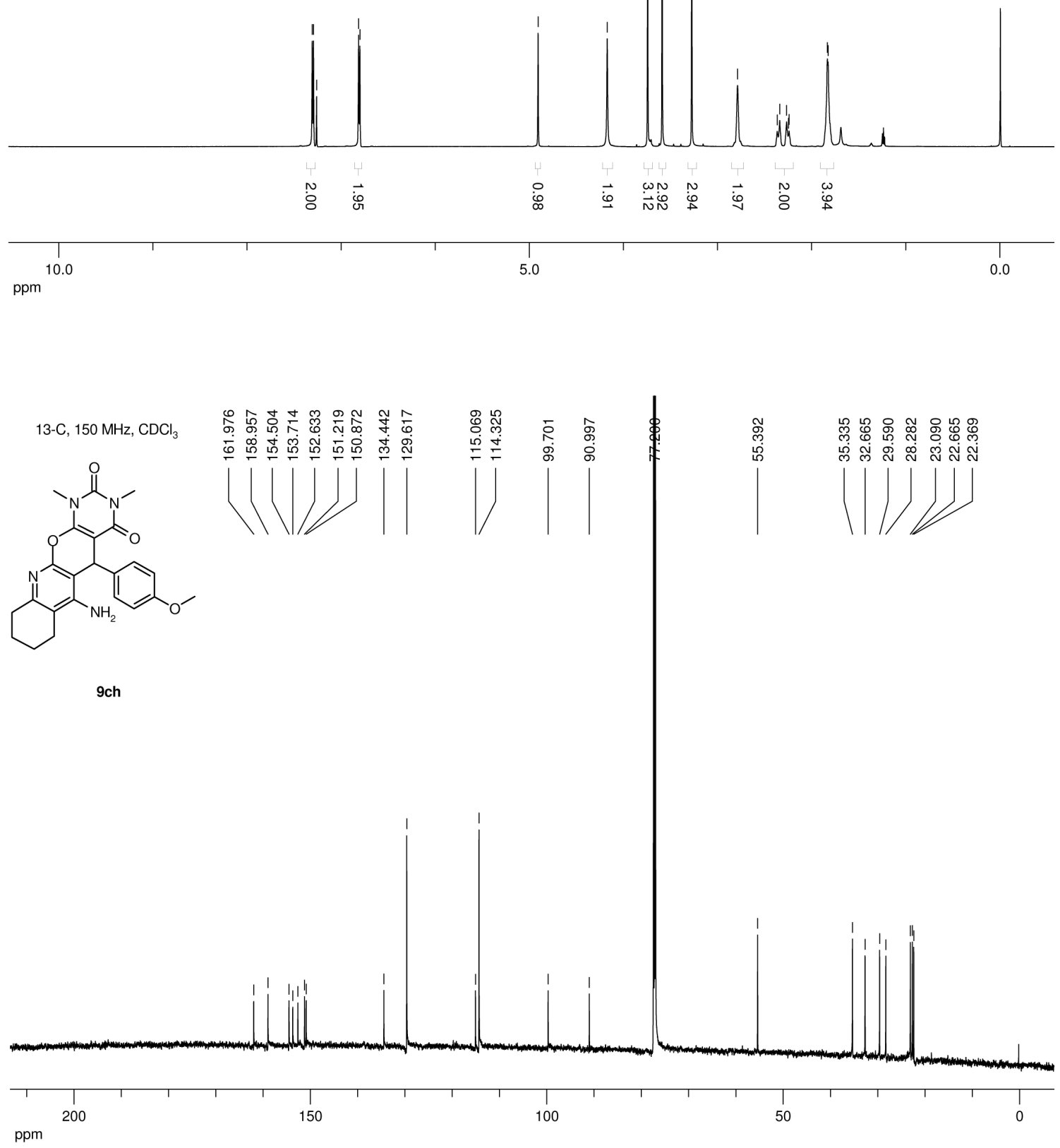


\section{nmr of products 11}
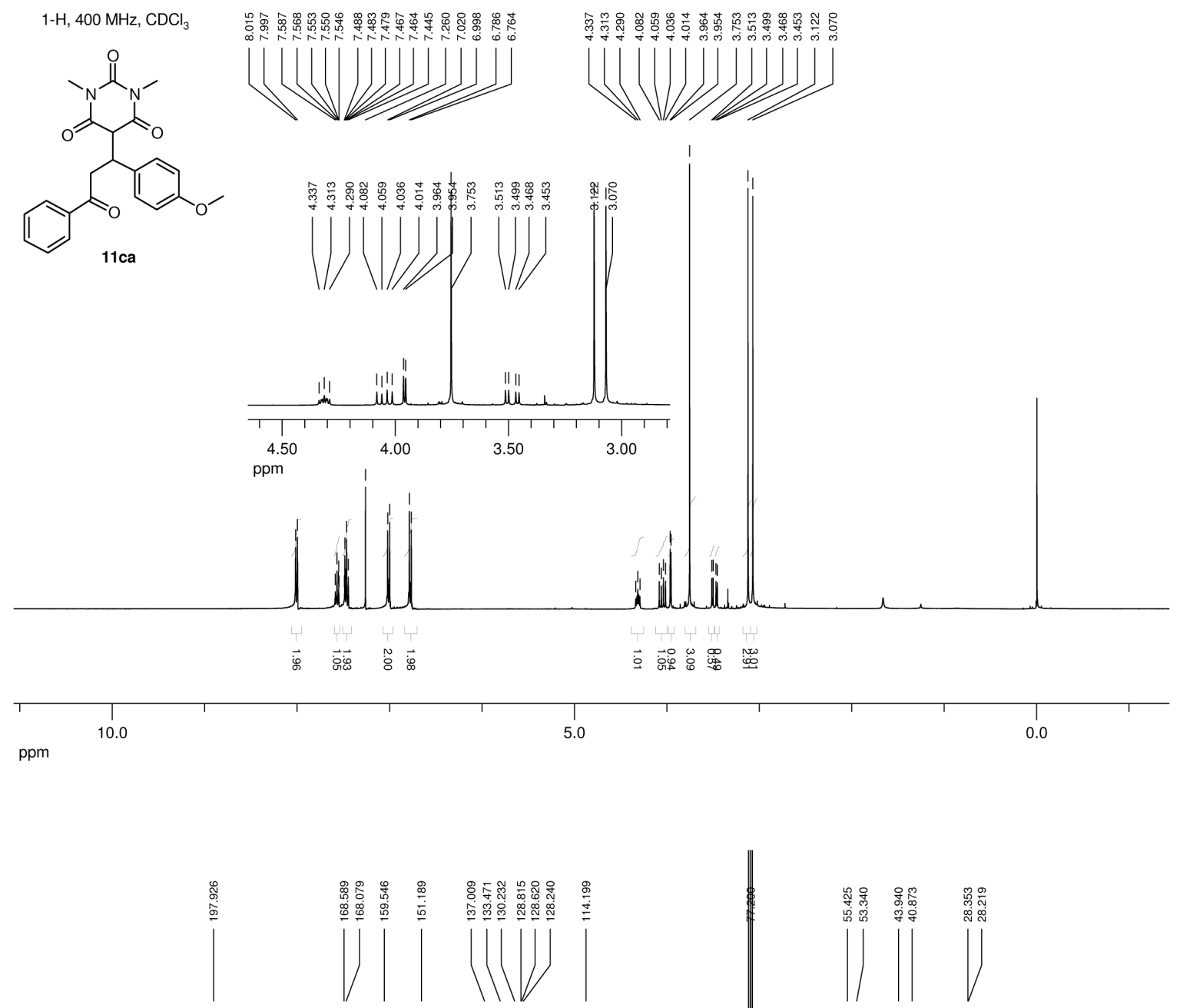

13-C, $75 \mathrm{MHz}, \mathrm{CDCl}_{3}$
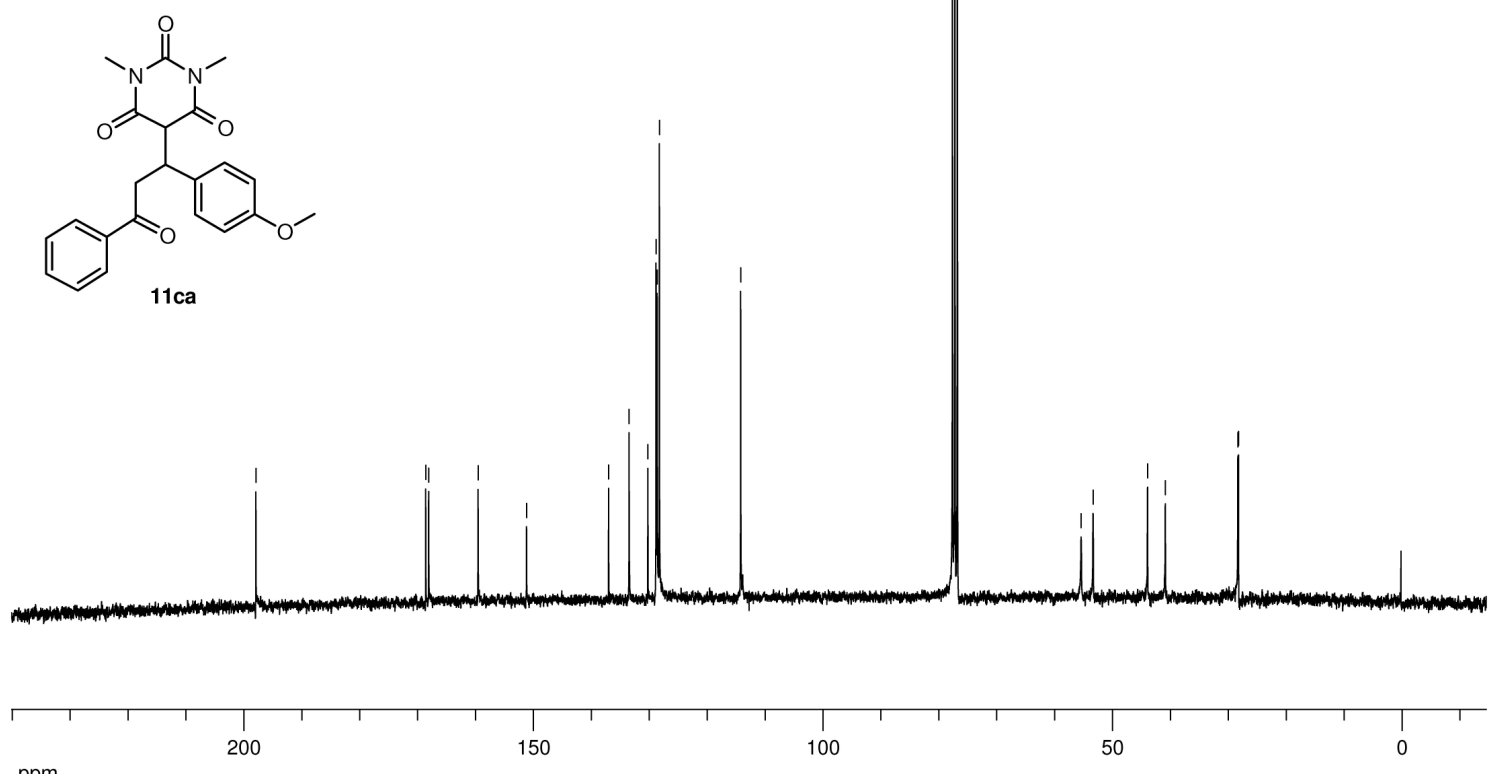

ppm 
S36
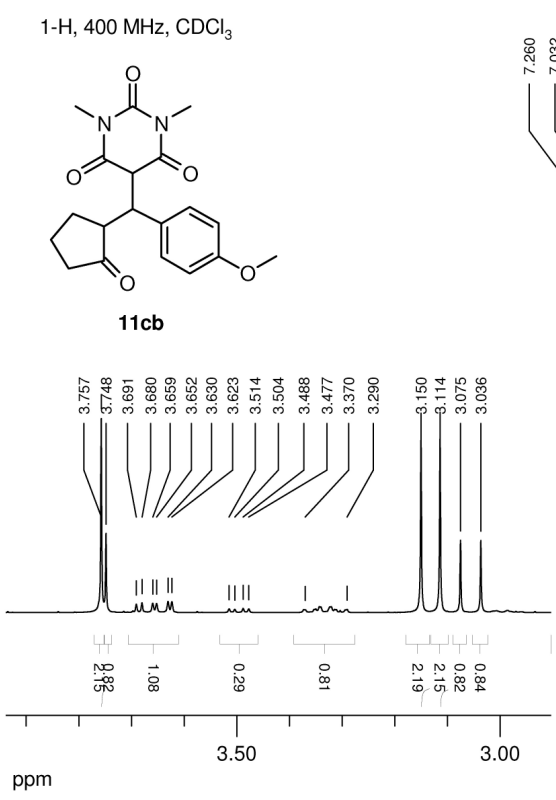

ppm
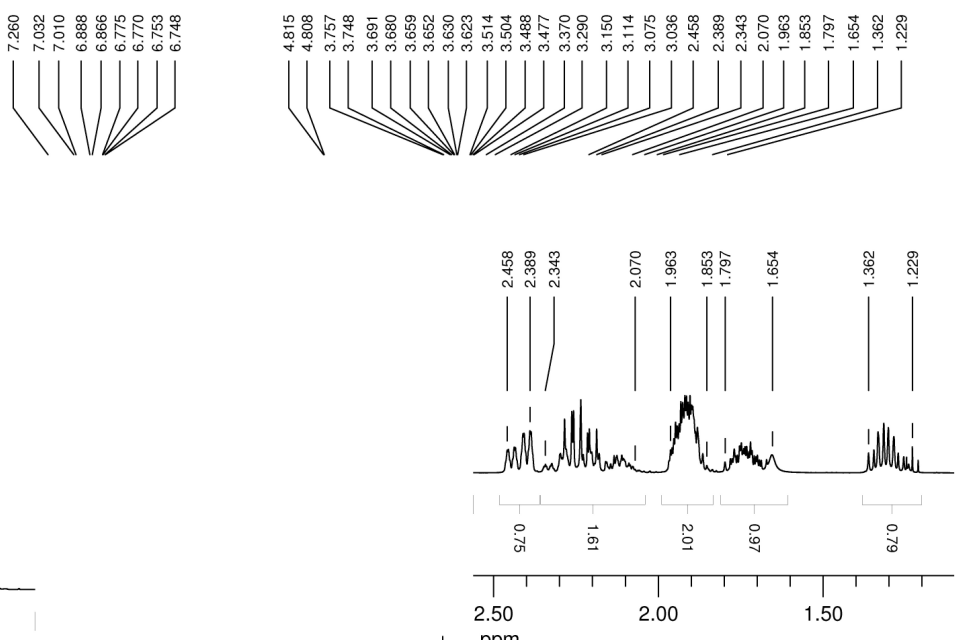
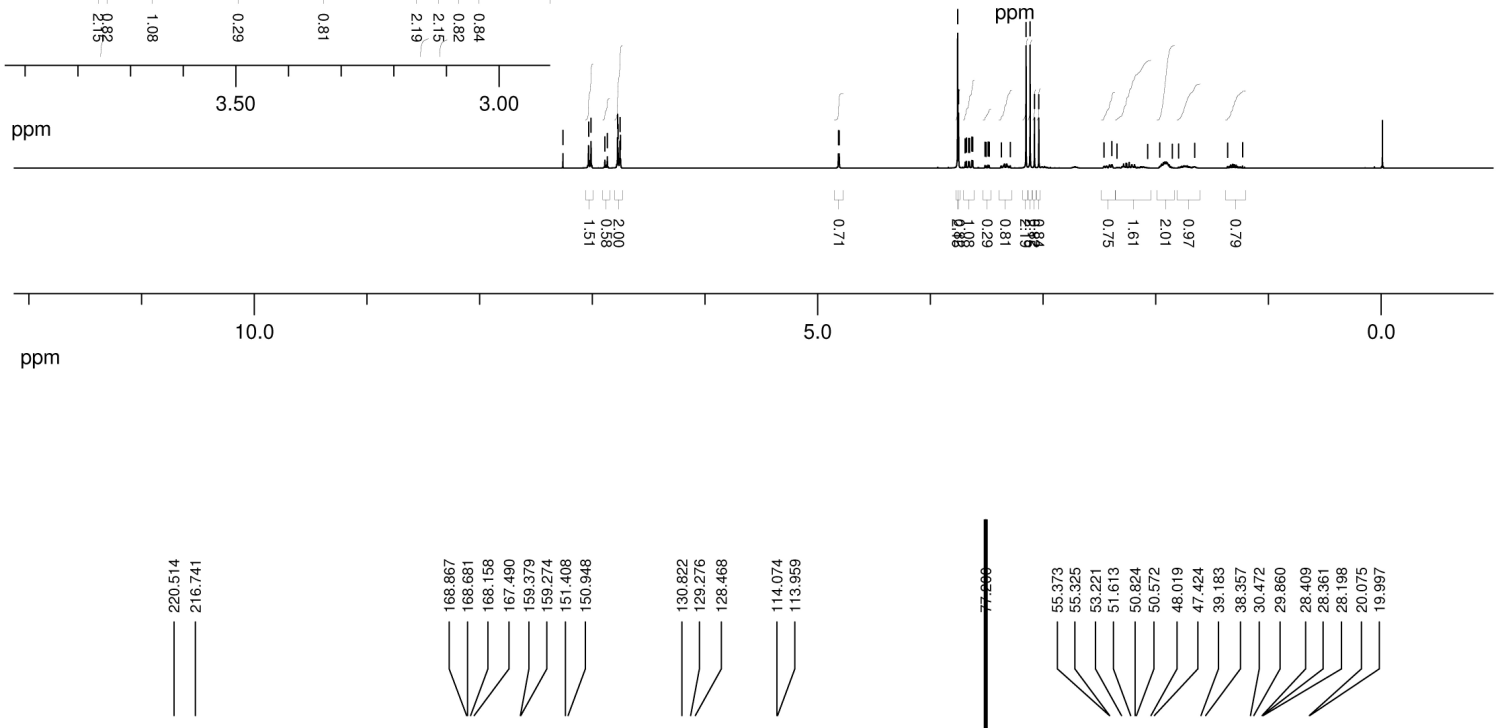

13-C, $150 \mathrm{MHz}, \mathrm{CDCl}_{3}$
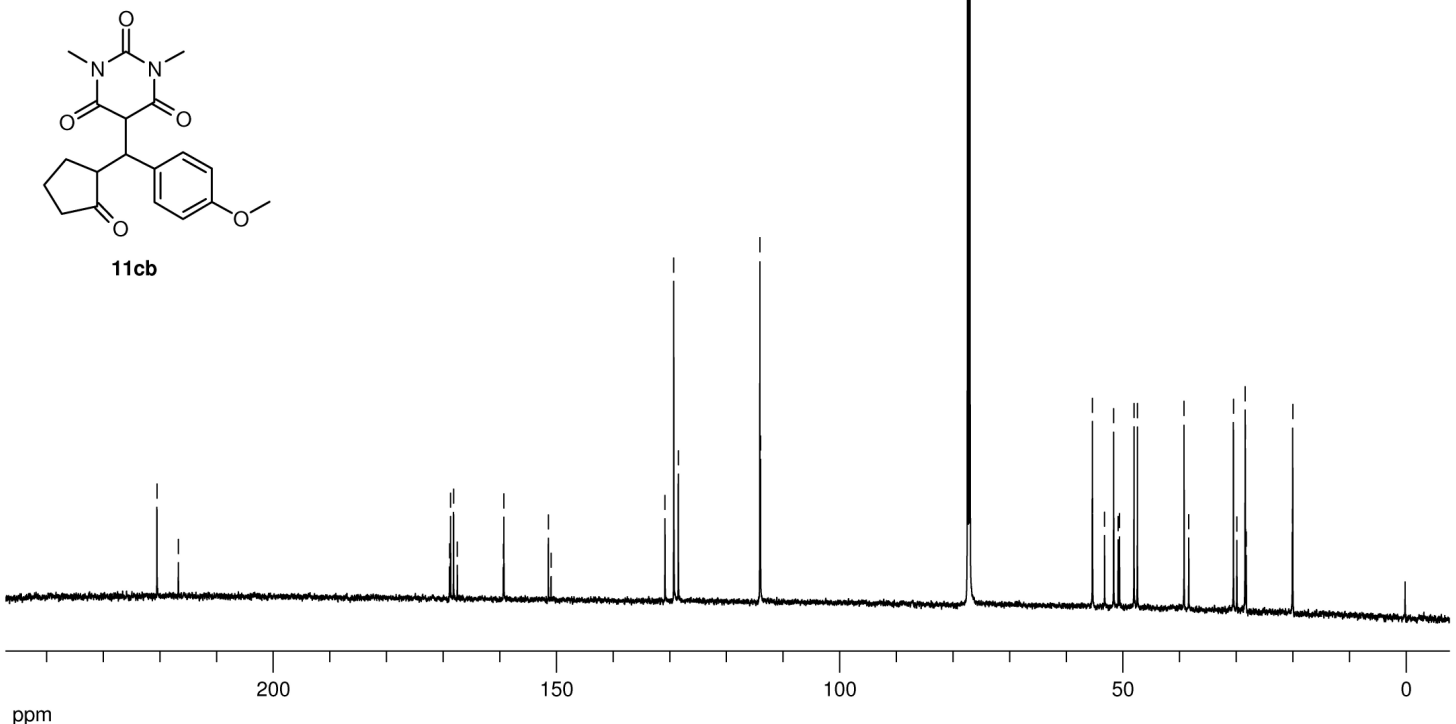
S37
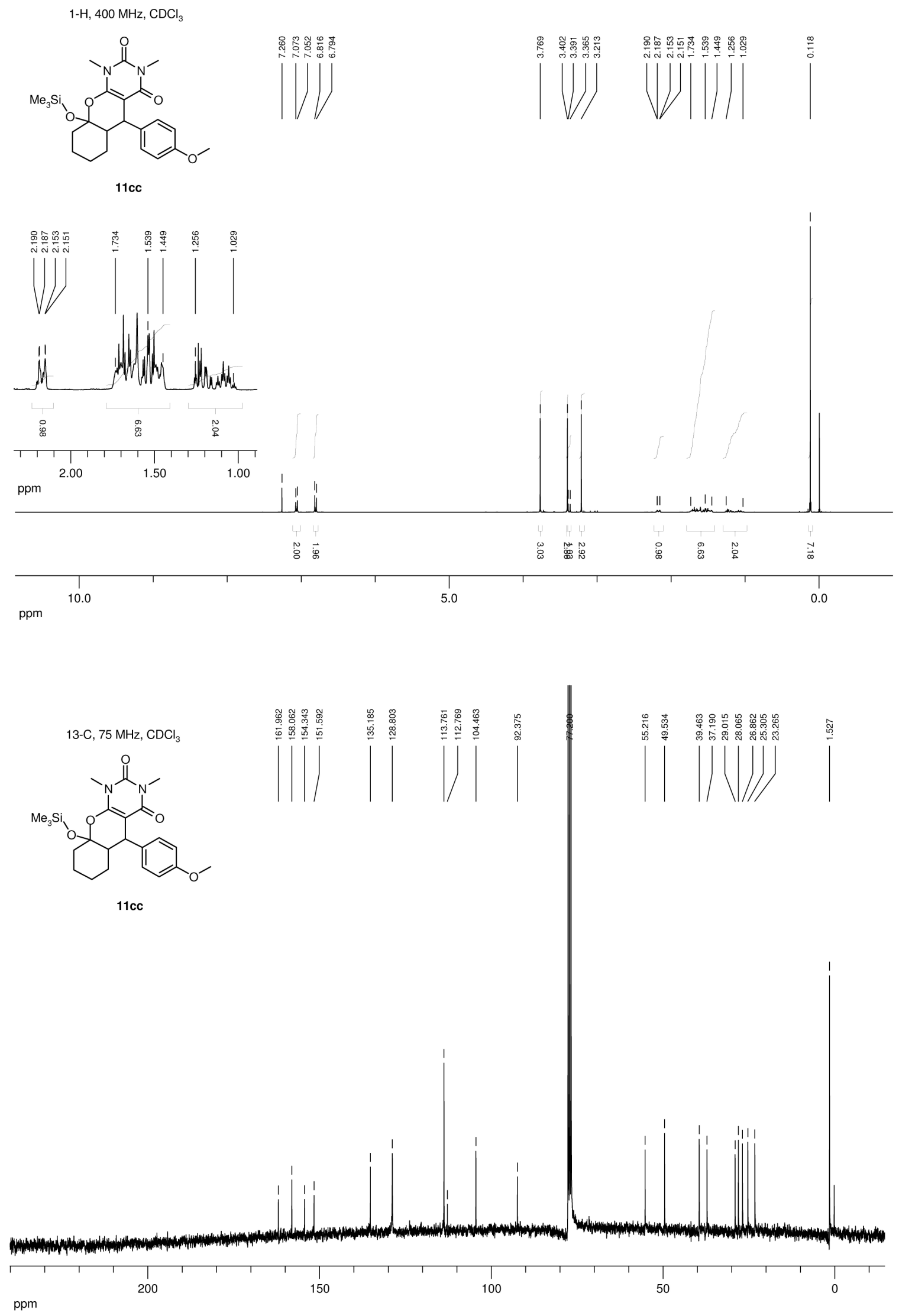
nmr of 12ce
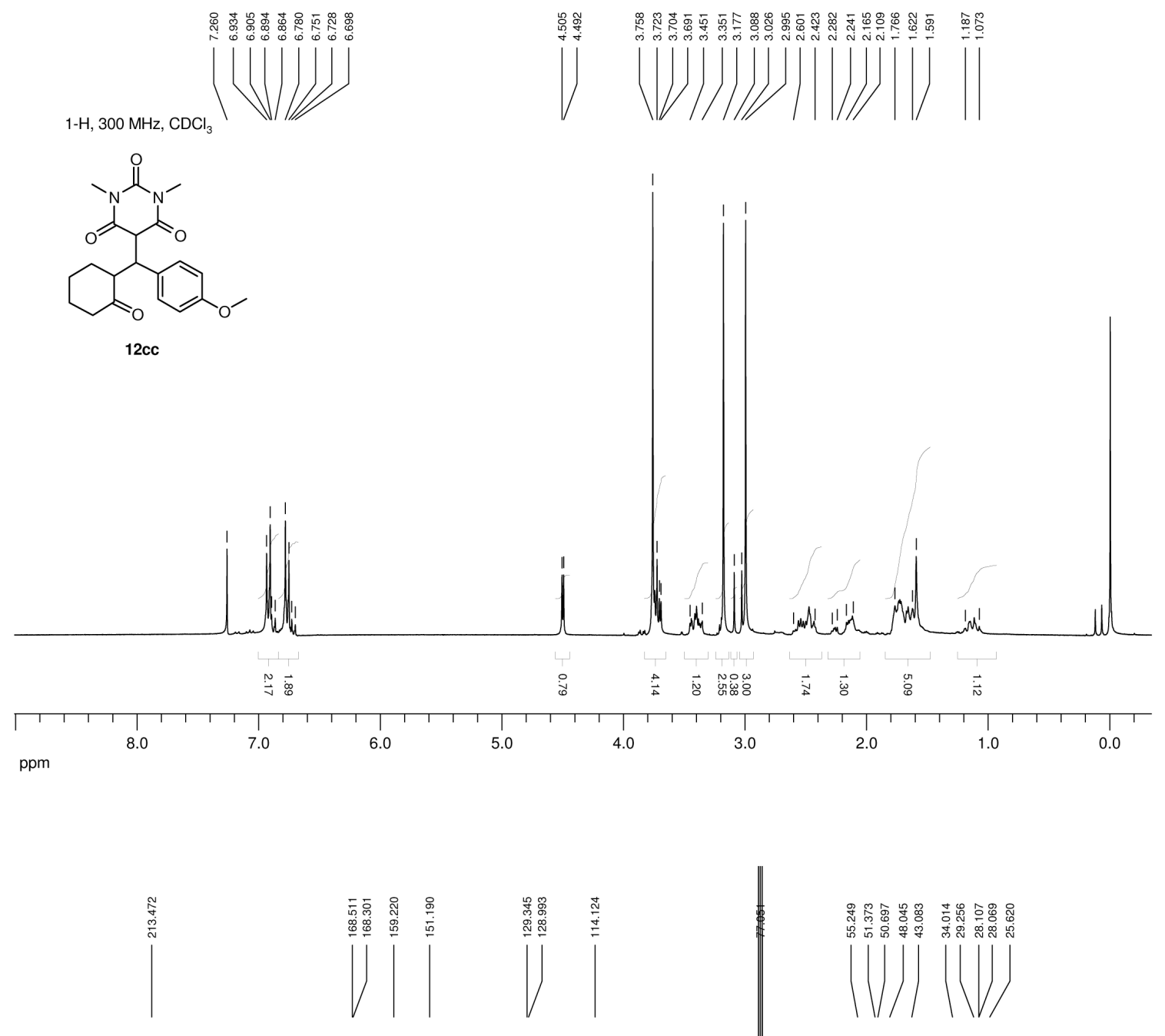

13-C, $75 \mathrm{MHz}, \mathrm{CDCl}$
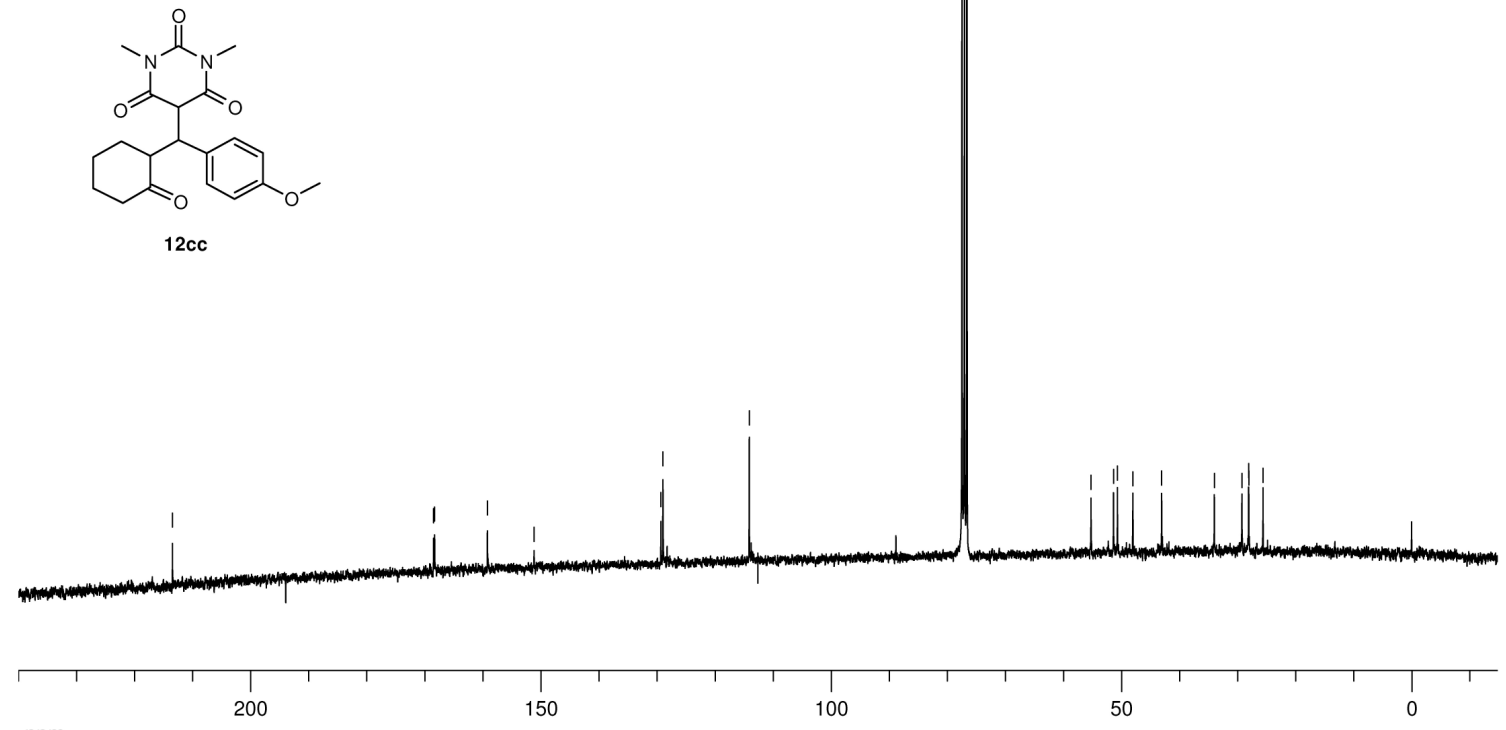

ppm 


\section{nmr of 14ca}

1- $\mathrm{H}, 400 \mathrm{MHz}, \mathrm{CDCl}_{3}$
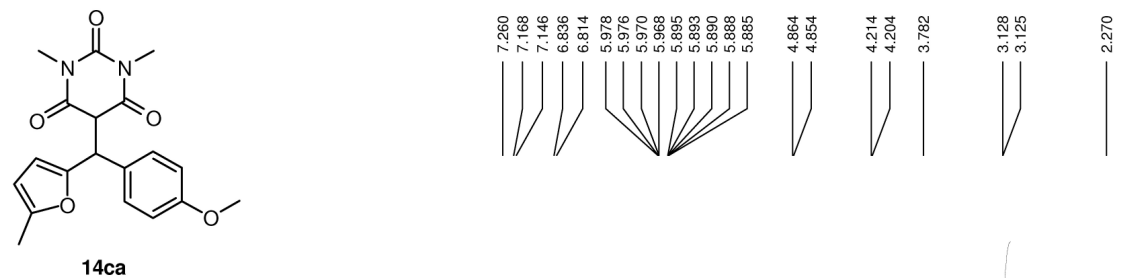

$14 \mathrm{c}$
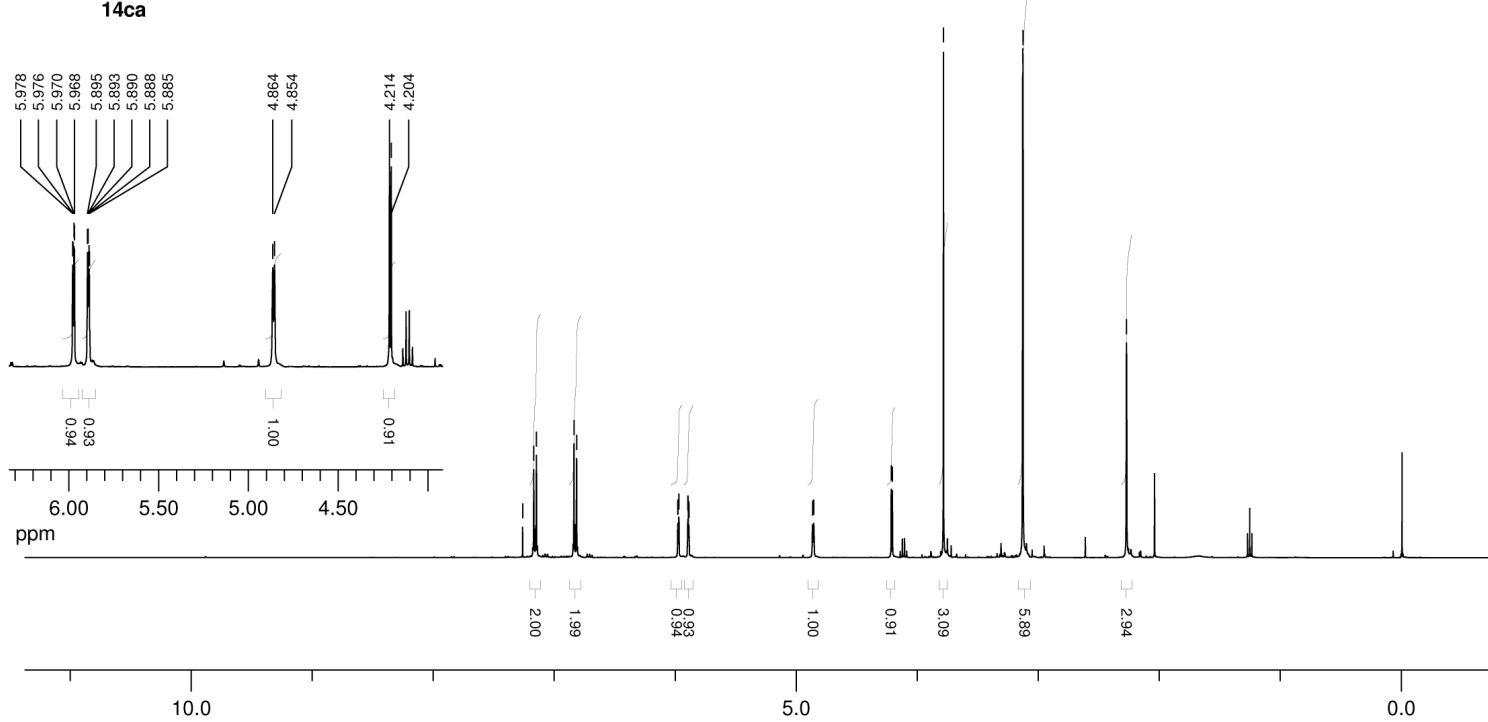

ppm
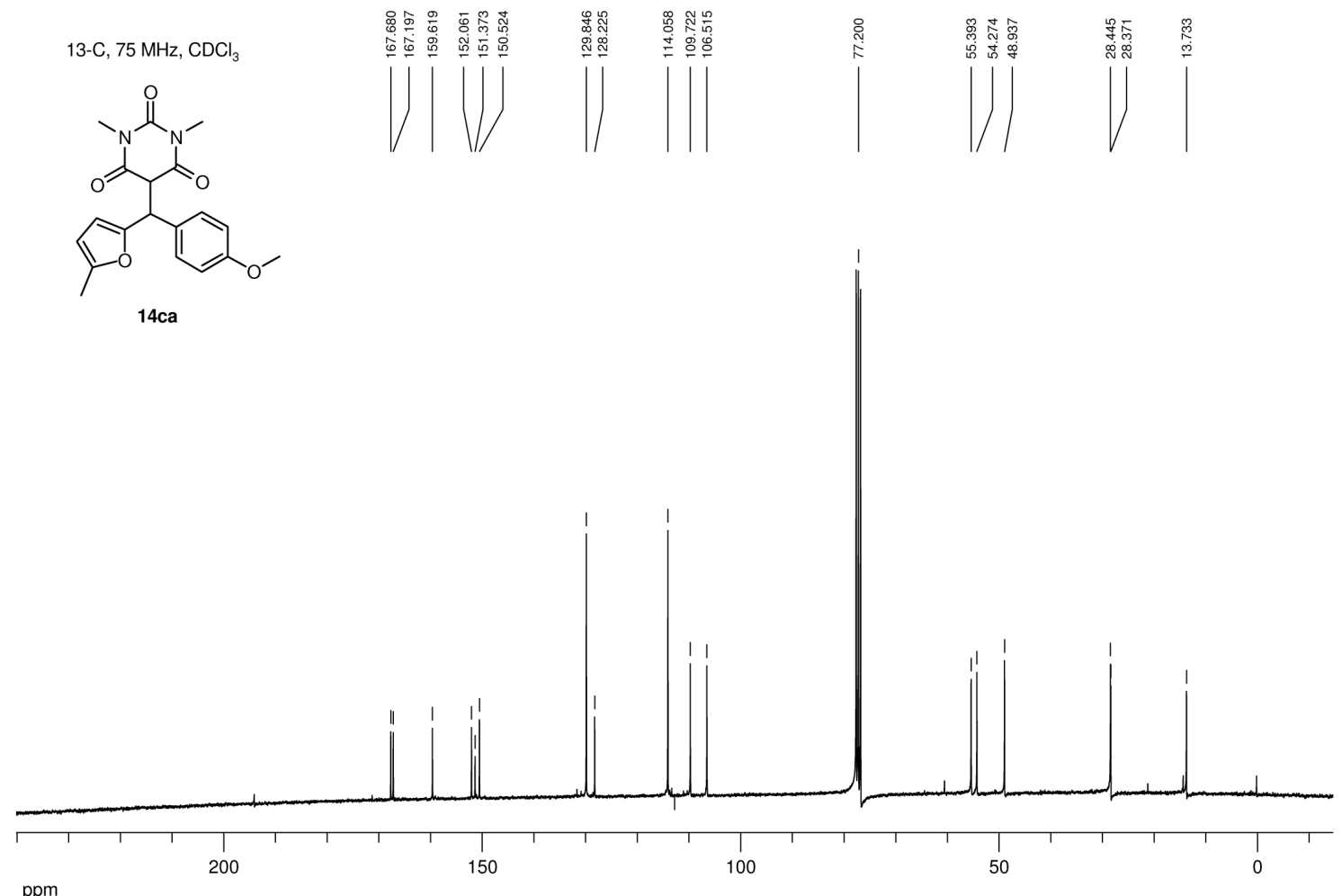


\section{X-ray Crystallography}

Data for the crystal structure determinations were collected on a Nonius ENRAF-CAD4 diffractometer. The SHELXS86 software was used to determine the structures, and the refinement was performed using the SHELXS93 software.

\begin{tabular}{|c|c|c|}
\hline Compound & $4 \mathrm{~cm}$ & $11 \mathrm{cc}$ \\
\hline Empirical formula & $\mathrm{C}_{17} \mathrm{H}_{21} \mathrm{~N}_{3} \mathrm{O}_{6}$ & $\mathrm{C}_{23} \mathrm{H}_{32} \mathrm{~N}_{2} \mathrm{O}_{5} \mathrm{Si}$ \\
\hline $\mathrm{FW}$ & 363.37 & 444.60 \\
\hline Crystal size (nm) & $0.23 \times 0.43 \times 0.53$ & $0.13 \times 0.47 \times 0.53$ \\
\hline Crystal system & triclinic & monoclinic \\
\hline Space group & $\mathrm{P} \overline{1}$ & $\mathrm{P} 2{ }_{1} / \mathrm{c}$ \\
\hline$a(\AA)$ & $8.3157(10)$ & $16.9134(35)$ \\
\hline$b(\AA)$ & $9.2369(14)$ & $7.8644(11)$ \\
\hline$c(\AA)$ & $12.748(2)$ & $18.6363(22)$ \\
\hline$\alpha\left(^{\circ}\right)$ & $80.999(12)$ & $90.00(0)$ \\
\hline$\beta\left(^{\circ}\right)$ & $71.729(11)$ & $95.354(12)$ \\
\hline$\gamma\left({ }^{\circ}\right)$ & $75.395(12)$ & $90.00(0)$ \\
\hline$V\left(\AA^{3}\right)$ & $896.9(2)$ & $2468.1(7)$ \\
\hline$Z$ & 2 & 4 \\
\hline$\rho_{\text {calcd }}\left(\mathrm{g} \mathrm{cm}^{-3}\right)$ & 1.346 & 1.197 \\
\hline$\mu\left(\mathrm{mm}^{-1}\right)$ & 0.103 & 0.129 \\
\hline$F(000)$ & 384 & 952 \\
\hline Temperature $(\mathrm{K})$ & $295(2)$ & $295(2)$ \\
\hline$\theta$ range (degrees) & $2.64-23.97$ & $2.41-23.97$ \\
\hline \multirow[t]{3}{*}{ Index ranges } & $0 \leq h \leq 9$ & $-19 \leq h \leq 0$ \\
\hline & $-10 \leq k \leq 10$ & $0 \leq k \leq 8$ \\
\hline & $-13 \leq l \leq 14$ & $-21 \leq l \leq 21$ \\
\hline No. of reflns measd & 2816 & 3850 \\
\hline No. of indep reflns with $I>2 \sigma(\mathrm{I})$ & 2084 & 2693 \\
\hline No. of parameters & 240 & 312 \\
\hline$R 1$ (obs. data) & 0.0486 & 0.0486 \\
\hline$w R 2$ (obs. data) & 0.01160 & 0.1242 \\
\hline$R 1$ (all data) & 0.0701 & 0.0766 \\
\hline$w R 2$ (all data) & 0.1313 & 0.1456 \\
\hline GooF on $F^{2}$ & 1.082 & 1.076 \\
\hline Resid. Electron density $\left(\mathrm{e} \AA^{-3}\right)$ & $+0.171 /-0.159$ & $+0.181 /-0.341$ \\
\hline
\end{tabular}

Crystallographic data of $\mathbf{4 c m}$ and 11cc have also been deposited with the Cambridge Crystallographic Data Center as supplementary publication no. CCDC-279759 for $\mathbf{4 c m}$ and no. CCDC-280644 for 11cc. Copies of the data can be obtained free of charge on application to CCCD, 12 Union Road, Cambridge CB2 1EZ, UK (e-mail: deposit@ccdc.cam.ac.uk). 


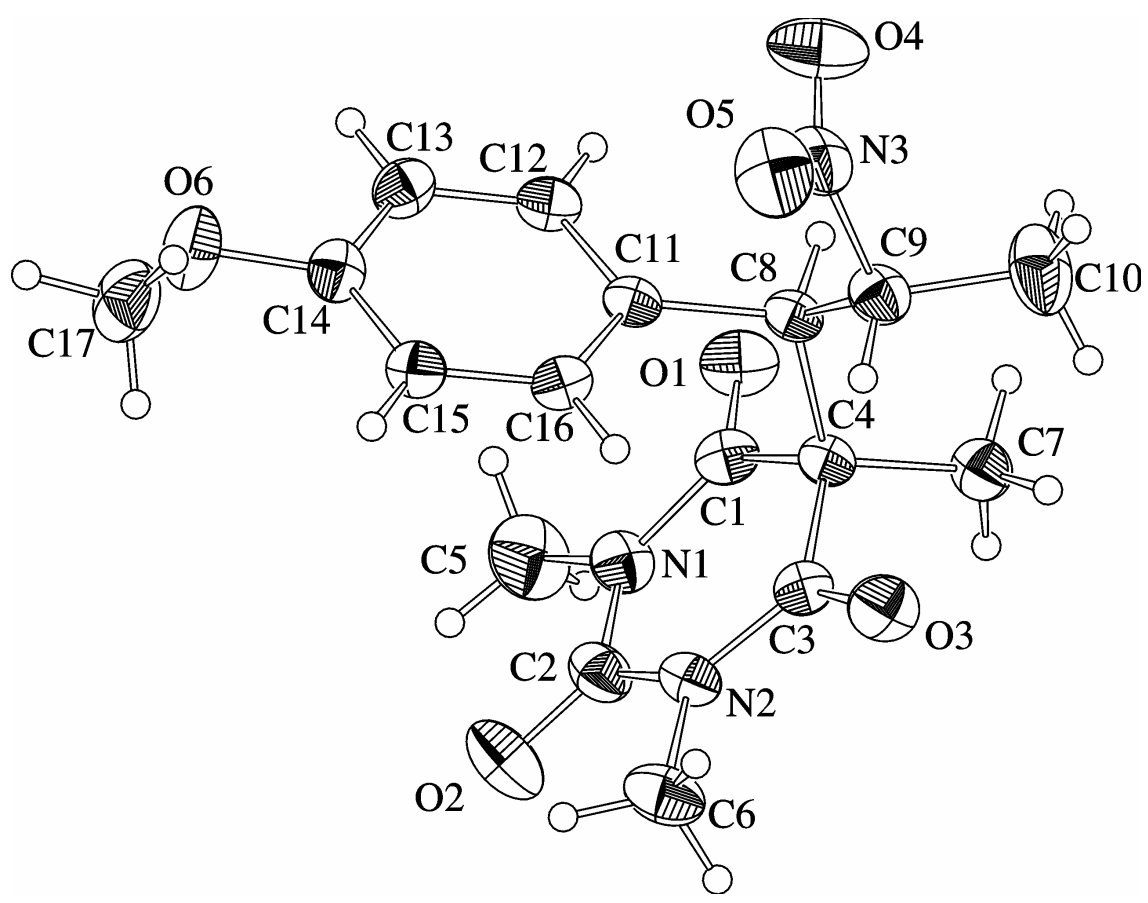

FIGURE: X-ray crystal structure (ORTEP projection) of one isomer of $\mathbf{4 c m}$.

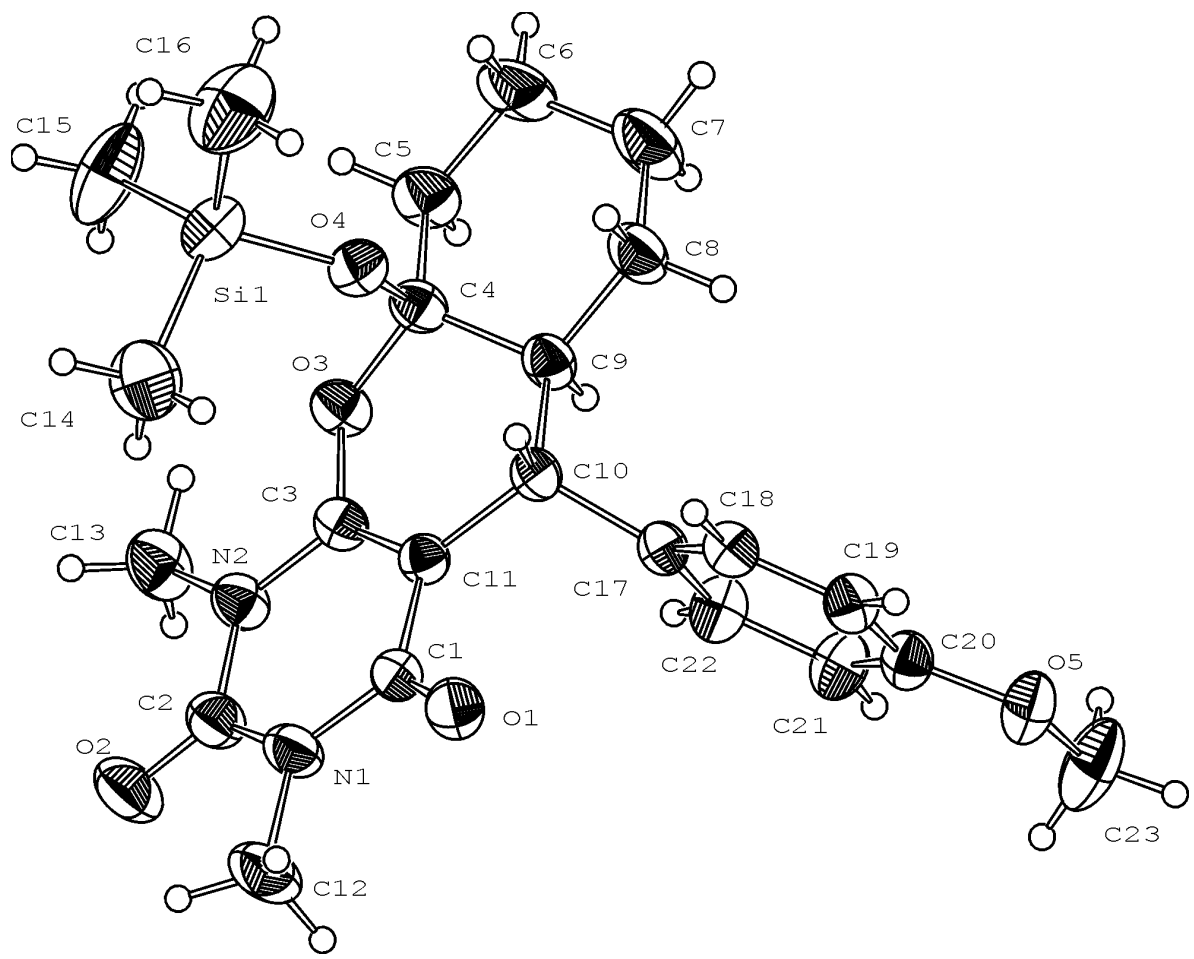

FIGURE: X-ray crystal structure (ORTEP projection) of 11cc. 